MARIA IZABEL ROMÃo LOPES

\title{
Validação para Língua Portuguesa da escala de graduação do paciente com hidrocefalia de pressão normal
}

Dissertação apresentada à Faculdade de Medicina da Universidade de São Paulo para obtenção do título de Mestre em Ciências.

Programa de Neurologia

Orientador: Prof. Dr. Fernando Campos Gomes Pinto 


\section{Dados Internacionais de Catalogação na Publicação (CIP)}

Preparada pela Biblioteca da

Faculdade de Medicina da Universidade de São Paulo

Creprodução autorizada pelo autor

Lopes, Maria Izabel Romão

Validação para Língua Portuguesa da escala de graduação do paciente com hidrocefalia de pressão normal/Maria Izabel Romão Lopes. -- São Paulo, 2013.

Dissertação(mestrado)--Faculdade de Medicina da Universidade de São Paulo.

Programa de Neurologia.

Orientador: Fernando Campos Gomes Pinto.

Descritores: 1.Hidrocefalia de pressão normal/patologia 2.Tradução 3.Escalas 4.Estudos de validação 5.Questionários 6.Índice de gravidade da doença

7.Transtornos neurológicos da marcha 8.Apraxia da marcha 9.Equilíbrio postural 10.Incontinência urinária/complicações 11.Demência/complicações

USP/FM/DBD-332/13 
Inicio meus agradecimentos a todos que, de alguma forma, passaram pela minha vida e participaram da construção de quem sou hoje. Agradeço, em especial, a algumas pessoas que contribuíram para execução direta desse trabalho.

Ao meu orientador, Prof. Dr. Fernando Campos Gomes Pinto, pela sua exemplar orientação, sem cobranças, mas carregada de compartilhamento e pelo seu inestimável valor pessoal. Ensinou-me que podemos alcançar nossos sonhos com paciência e determinação.

A minhas amigas Fernanda Letskake e Juliana Tornai, pelas experiências divididas, pela amizade e pelo companheirismo nos anos em que passamos juntas.

À querida amiga Wendry Paixão, pela força recebida, extensa sabedoria e sua ajuda, sem a qual não conseguiria concluir esse trabalho.

Aos Professores Doutores Edson Shu, Ricardo Botelho e Koshiro Nishikuni, pelas valiosas contribuições durante o exame de qualificação desse trabalho.

A minha família, em especial, a minha mãe Lucimary, a meu avô Deodato e a minha tia Claudimary, pelos quais eu sinto imenso amor, orgulho e carinho. Por estarem sempre ao meu lado, com incentivo e por estimular meu desenvolvimento. Saudades de minha tia Mary Lucia, que carrego no coração. A toda minha família, a minhas adoradas primas Daniela e Carolina, pela alegria e mesmo, às vezes, longe, se fazem presentes em todos os momentos. Sou muito feliz de tê-los em minha vida!

E por fim, um achado precioso em meu caminho, Leandro Maia, pela paciência, pelo companheirismo e pelo carinho. Espero poder retribuir com toda a felicidade que merece! 
Não poderia finalizar sem agradecer a Deus, pela Sua grandeza, pelo Seu amor incondicional. Obrigado por nunca desistir de mim, por me amparar quando eu mais preciso e por me mostrar que o melhor sempre está por vir. 
“A grandeza não consiste em receber honras, mas em merecê-las".

Aristóteles 


\section{Normalização Adotada}

Esta tese está de acordo com as seguintes normas, em vigor no momento desta publicação:

Referências: adaptado de International Committee of Medical Journals Editors (Vancouver).

Universidade de São Paulo. Faculdade de Medicina. Divisão de Biblioteca e Documentação. Guia de apresentação de dissertações, teses e monografias. Elaborado por Anneliese Carneiro da Cunha, Maria Julia de A. L. Freddi, Maria F. Crestana, Marinalva de Souza Aragão, Suely Campos Cardoso, Valéria Vilhena. 3a ed. São Paulo: Divisão de Biblioteca e Documentação; 2011.

Abreviaturas dos títulos dos periódicos de acordo com List of Journals Indexed in Index Medicus. 


\section{LISTA DE FIGURAS}

\section{LISTA DE TABELAS}

\section{LISTA DE GRÁFICOS}

\section{LISTA DE SIGLAS E ABREVIATURAS}

\section{RESUMO}

\section{SUMMARY}

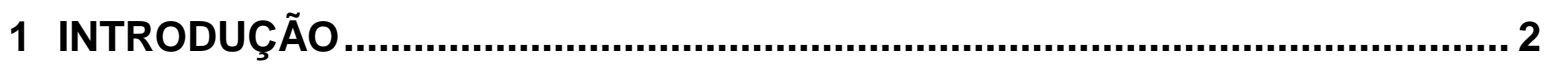

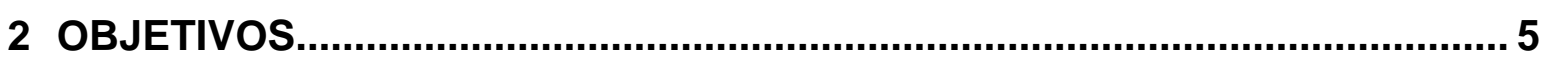

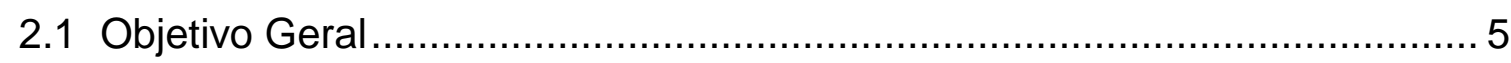

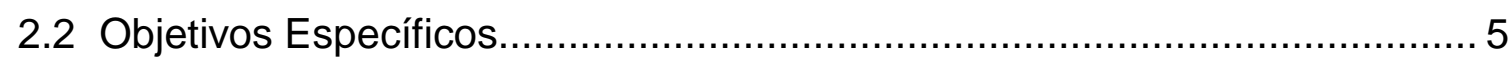

3 REVISÃO DA LITERATURA ................................................................... 7

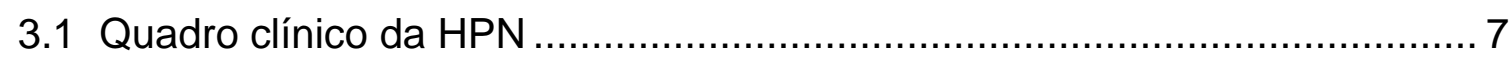

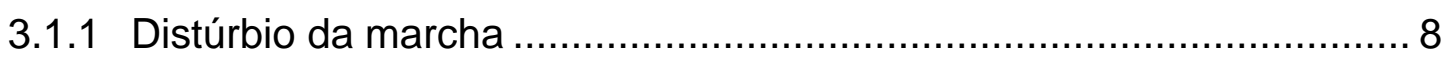

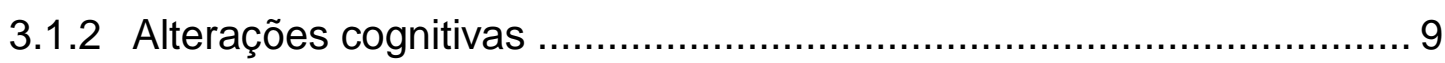

3.1.3 Incontinência urinária ................................................................. 10

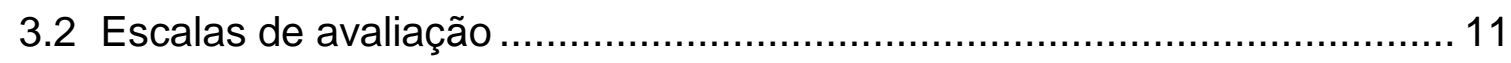

3.2.1 Medida de independência funcional ........................................... 12

3.2.2 Escala de equilíbrio de Berg..................................................... 14

3.2.3 Índice de marcha dinâmica............................................................... 14

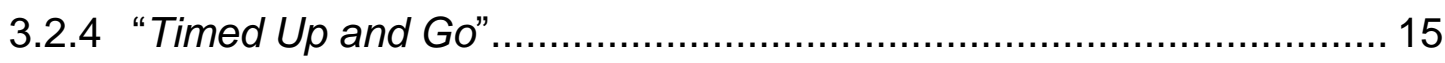

3.2.5 Escalas de graduação do paciente com HPN ……………….......... 16

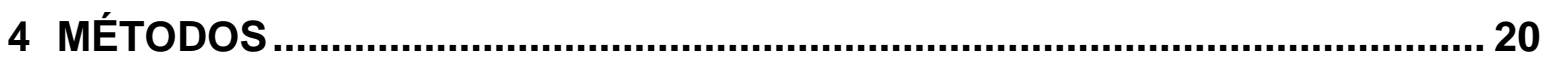

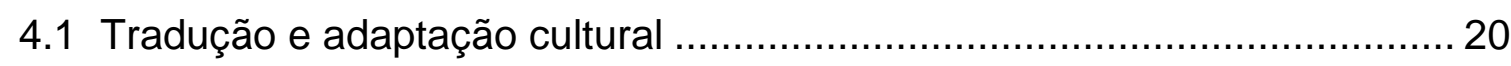

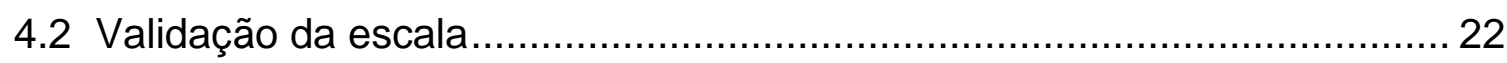

4.2.1 Medida de Independência Funcional - MIF ...................................... 23

4.2.2 Teste de Equilíbrio de Berg (Berg) ................................................ 24

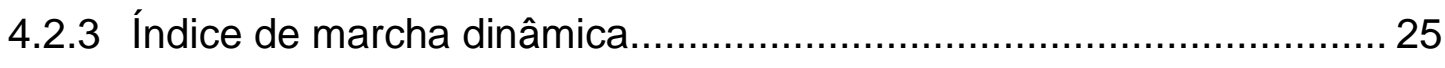

4.2.4 "Timed Up and Go" (TUG) ..................................................... 26

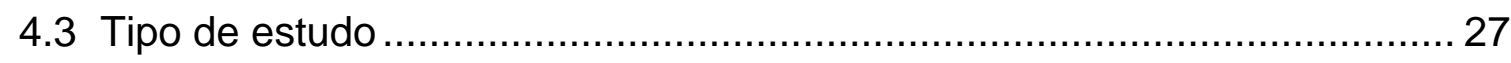

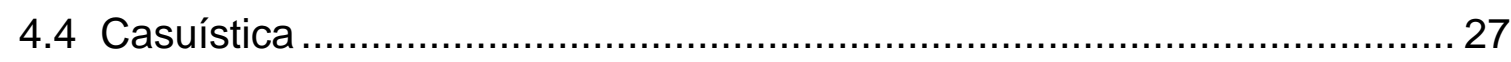

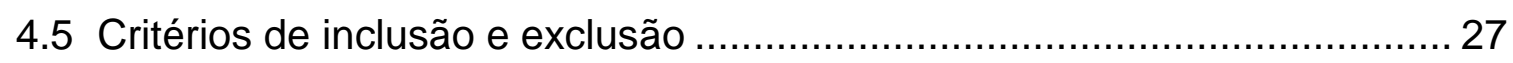


5 RESULTADOS

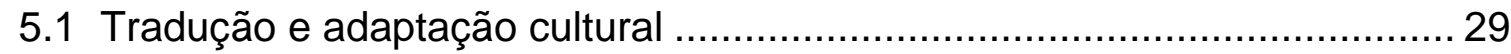

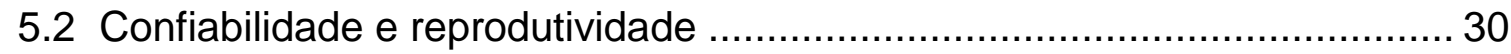

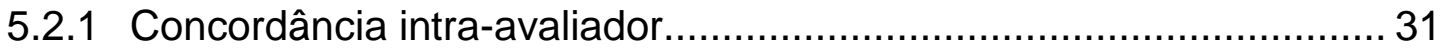

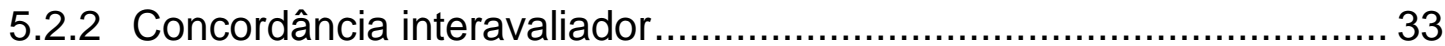

5.3 Comparações dos valores da escala de graduação HPN com demais escalas selecionadas.

5.3.1 Comparação dos valores HPN com a independência funcional

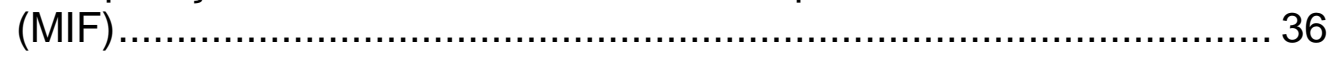

5.3.2 Comparação dos valores HPN com a escala de BERG ..................... 37

5.3.3 Comparação dos valores HPN com a marcha dinâmica (DGl) .......... 39

5.3.4 Comparação dos valores HPN com "Timed Up and Go" (TUG)........ 40

5.4 Correlações entre escalas sobre item incontinência urinária...................... 42

5.5 Correlações entre escalas sobre item cognição ...................................... 43

5.6 Correlações entre escalas sobre item marcha........................................ 45

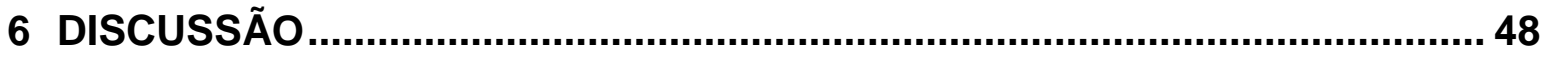

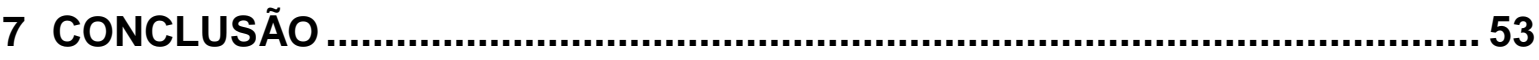

8 ANEXOS

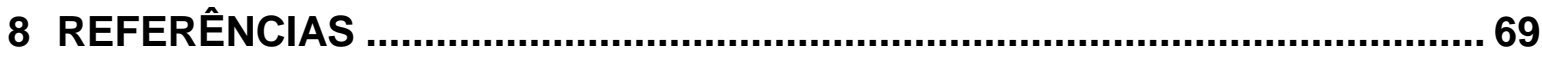

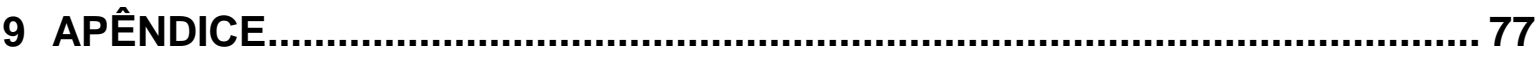


Figura 1 Dimensões e pontuações da MIF 13

Figura 2 Sequencia da tradução da escala.................................................... 21

Figura 3 Sequencia da avaliação funcional................................................. 23 
Tabela 1 Categorias e escores da Escala de Graduação do paciente com HPN, coletados durante as avaliações.

Tabela 2 Categorias e escores da escala medida de independência funcional, coletados durante as avaliações.

Tabela 3 Categorias e escores do teste de equilíbrio de Berg, coletados durante as avaliações.

Tabela 4 Categorias e escores do teste Índice de marcha dinâmica, coletados durante as avaliações............................................. 26

Tabela 5 Categorias e escores do TUG....................................... 26

Tabela 6 Escala de Graduação de HPN.......................................... 29

Tabela 7 Características dos 121 pacientes segundo faixa etária e gênero, que foram avaliados no Ambulatório do Grupo de Hidrodinâmica Cerebral do IPQ-HCFMUSP, no período de junho de 2010 a

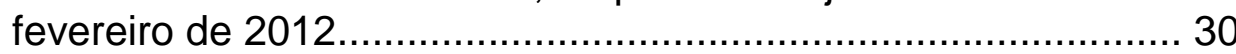

Tabela 8 Frequências absolutas e relativas das características relacionadas aos domínios da amostra....................................................... 30

Tabela 9 Interpretação da concordância segundo valores da medida de Kappa............................................................................... 31

Tabela 10 Valores de Kappa para as diferentes pontuações da escala....... 31

Tabela 11 Kappa geral do item marcha (intra-avaliador)........................... 32

Tabela 12 Valores de Kappa para as diferentes pontuações da escala....... 32

Tabela 13 Kappa geral do item demência (intra-avaliador)........................32

Tabela 14 Valores de Kappa para as diferentes pontuações da escala.......33

Tabela 15 Kappa geral do item incontinência (intra-avaliador)....................33

Tabela 16 Valores de Kappa para as diferentes pontuações........................33

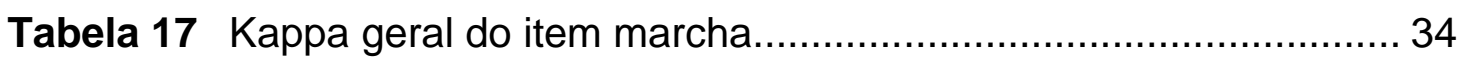

Tabela 18 Valores de Kappa para as diferentes pontuações........................34

Tabela 19 Kappa geral do item demência (interavaliador) ......................... 34 
Tabela 20 Valores de Kappa para as diferentes pontuações.

Tabela 21 Kappa geral do item incontinência urinária............................ 35

Tabela 22 Correlações segundo valor de Pearson................................. 35

Tabela 23 Comparação dos valores totais das escalas HPN e MIF, por meio da Correlação de Pearson................................................. 36

Tabela 24 ANOVA, com tabela de médias e desvios-padrão........................ 36

Tabela 25 Comparações múltiplas de Tukey............................................. 37

Tabela 26 Comparação dos valores das escalas HPN e BERG, por meio da Correlação de Pearson............................................................ 38

Tabela 27 ANOVA, com tabela de médias e desvios-padrão..................... 38

Tabela 28 Comparações múltiplas de Tukey....................................... 38

Tabela 29 Comparação dos valores das escalas HPN e DGI, por meio da Correlação de Pearson........................................................... 39

Tabela 30 Teste t-independente, com tabela de médias e desvios-padrão..40

Tabela 31 Comparação dos valores da escala HPN e TUG, por meio da Correlação de Pearson.......................................................... 41

Tabela 32 ANOVA, com tabela de médias e desvios-padrão...................... 41

Tabela 33 Comparações múltiplas de Tukey........................................ 41

Tabela 34 Comparação apenas dos valores dos itens relacionados à continência das escalas HPN e MIF, por meio da Correlação de Pearson.

Tabela 35 Comparação descritiva segundo os valores dos itens de continência das escalas HPN e MIF....

Tabela 36 Comparação apenas dos valores dos itens relacionados à cognição da MIF com item demência da HPN, por meio da Correlação de Pearson.

Tabela 37 Comparação descritiva segundo os valores dos itens relacionados à cognição da HPN e MIF.

Tabela 38 Comparação apenas dos valores dos itens relacionados à marcha da MIF com item marcha da HPN, por meio da Correlação de Pearson.

Tabela 39 Comparação descritiva segundo os valores dos itens relacionados à marcha da HPN e MIF. 45 
Gráfico 1 Demonstra pontuações da MIF, segundo HPN...........................37

Gráfico 2 Demonstra pontuações da BERG, segundo HPN......................39

Gráfico 3 Demonstra pontuações da DGI, segundo HPN.........................40

Gráfico 4 Demonstra valores do TUG, segundo HPN............................ 42 


\begin{tabular}{ll} 
ANOVA & Análise de Variância \\
AVD & Atividades de Vida Diária \\
BERG & Teste de Equilíbrio de Berg \\
CAPPesq & Comissão de Ética para Análise de Projetos de Pesquisa \\
DGI & Índice de Marcha Dinâmica \\
DVP & Derivação Ventriculoperitonial \\
HCFMUSP & $\begin{array}{l}\text { Hospital das Clínicas da Faculdade de Medicina da Universidade } \\
\text { de São Paulo }\end{array}$ \\
HPN & Hidrocefalia de Pressão Normal \\
ICIQ & Questionário Internacional de Incontinência \\
IPQ & Instituto de Psiquiatria \\
IU & Incontinência Urinária \\
LCR & Líquido Cefalorraquidiano \\
MIF & Medida de Independência Funcional \\
MMSE & Mini Exame do Estado Mental \\
NPH & Normal Pressure Hydrocephalus \\
SVE & Encefalopatia Vascular Subcortical \\
TCLE & Termo de Consentimento Livre e Esclarecido \\
TUG & "Timed Up and Go” \\
TVE & Terceiro-Ventriculostomia Endoscópica \\
\hline
\end{tabular}




\section{Resumo}

Lopes MIR. Validação para Língua Portuguesa da escala de graduação do paciente com hidrocefalia de pressão normal [Dissertação]. São Paulo: Faculdade de Medicina, Universidade de São Paulo; 2013. p 75.

O presente estudo validou para a Língua Portuguesa a escala de graduação do paciente com hidrocefalia de pressão normal (HPN) desenvolvida na Língua Inglesa como "Grading scale for idiopathic normal pressure hydrocephalus". Duas traduções independentes da escala de HPN foram feitas por médicos brasileiros, fluentes na Língua Inglesa. Após harmonização dessas, a tradução resultante foi retrotraduzida independentemente por dois outros médicos, que desconheciam a escala original. Seguiu-se a última tradução e revisão para a Língua Portuguesa por uma profissional tradutora da área da saúde. A comparação da versão final traduzida com a escala original foi realizada pelo comitê multiprofissional que não estava envolvido no processo de tradução, pontuando-se para item distúrbio de marcha: 0 ausente; 1 marcha instável, mas independente; 2 anda com um apoio; 3 anda com 2 apoios ou andador e 4 não é possível andar. No item demência: 0 ausente; 1 sem demência aparente, mas apático; 2 socialmente dependente, mas independente na residência; 3 parcialmente dependente na residência e 4 totalmente dependente. Incontinência Urinária: 0 ausente; 1 ausente, mas com polaciúria ou urgência miccional; 2 às vezes, apenas à noite e 4 frequente. Para obtenção da pontuação final, devem-se somar os itens, sendo que, quanto maior o escore final, maior comprometimento do paciente. O resultado foi pré-testado em um estudo-piloto. A versão final da escala de HPN para o Português, bem como as escalas de equilíbrio de Berg, índice de marcha dinâmica e "timed up and go" foram aplicadas simultaneamente em cento e vinte e um pacientes consecutivos com diagnóstico médico de hidrocefalia de pressão normal (setenta e três homens e quarenta e oito mulheres) que procuraram 0 Ambulatório de Hidrodinâmica Cerebral, da Divisão de Neurocirurgia Funcional do Instituto de Psiquiatria do Hospital das Clínicas de São Paulo da FMUSP, no período de julho de 2010 a março de 2012. Foram testadas as propriedades psicométricas do questionário, como confiabilidade e validade. A idade mediana foi de 71,09 anos (intervalo de 35 a 92 anos). O período médio de reteste para a escala de HPN foi de sete dias. Nenhuma alteração do formato original da escala foi observada no final do processo de tradução e adaptação cultural. O grau de concordância e reprodutividade foi alta, como demonstrado 
pela medida de concordância Kappa, com excelente correlação intraobservador para itens da escala de HPN individualmente avaliados: marcha $(0,8)$, demência $(0,90)$ e incontinência $(0,87)$. Na análise interobservador, o resultado foi excelente, com item marcha $(0,91)$, demência $(0,86)$ e incontinência $(0,87)$. A correlação entre a escala de HPN com as demais escalas foi considerada de moderada a satisfatória para a maioria dos itens, variando de -,069 a 0,55 na correlação de Pearson. A avaliação individual entre escalas sobre os itens incontinência urinária, demência e marcha foram também satisfatórias e estatisticamente significantes. A versão para o Português da escala de graduação do paciente com HPN foi traduzida e validada com sucesso para aplicação em pacientes brasileiros de ambos os sexos, apresentando satisfatória confiabilidade e validade.

Descritores: Hidrocefalia de pressão normal/patologia; Tradução; Escalas; Estudos de validação; Questionários; Índice de gravidade da doença; Transtornos neurológicos da marcha; Apraxia da marcha; Equilíbrio postural; Incontinência urinária/complicações; Demência/complicações. 
Lopes MIR. Validation to Portuguese Language of graduation scale for patients with normal pressure hydrocephalus [dissertation]. São Paulo: Faculdade de Medicina, Universidade de São Paulo; 2013. p. 75.

The current study validated to Portuguese language the graduation scale for patients with normal pressure hydrocephalus (NPH) developed on English language as "Grading scale for idiopathic normal pressure hydrocephalus". Two translations independent of NPH scale were done by Brazilian doctors, fluent on English language. After harmonization of both, the resulting translation was back-translated independently by two other doctors, that unaware to the original scale. Followed the last translation and revision to Portuguese language by a professional translator of health area. The comparison of last translated version with original scale was performed by one multiprofessional committee not involved on translation process. Was established specific punctuation to components of NPH classical triad, the punctuation to gait: 0 absent; 1 unstable gait, but independent; 2 walk with a support; 3 walk with 2 supports or walker an 4 is not possible to walk. On dementia item: 0 absent; 1 without apparent dementia, but apathetic; 2 socially dependent, but independent on resistance; 3 partial dependent on resistance and totally dependent. Urinary incontinence: 0 absent, 1 absent but with pollakisuria or urinary urgency, 2 sometimes, just at night; 3 sometimes even during the day and 4 frequently. The obtained final punctuation was given by summation of three items, and the higher the final punctuation, greater involvement of the patient. The result was pre-tested in a pilot study. The last version of NPH to Portuguese, as well the Berg balance scales, Dynamic Gait Index and "timed up and go" were applied simultaneously in 121 consecutive patients with medical diagnostic of normal pressure hydrocephalus (73 men and 48 women) who sought the Ambulatory of Cerebral Hydrodynamics, Division of Functional Neurosurgery, Institute of Psychiatry, Hospital das Clínicas in Sao Paulo from FMUSP, on period July / 2010 to march / 2012. The psychometric properties, reliability and validity of questionnaire were tested. The mean age was 71,09 years, ranging from 35 to 92 years. The period of mean retest was 7 days. None change to the original format of the scale was observed at the end of the translation process and cultural adaptation. The rate of agreement and reproducibility was high, as confirmed by measure of agreement of Kappa, with excellent intra-observer correlation for NPH scale items individually evaluated: gait $(0,80)$, dementia $(0,90)$ and incontinence $(0,87)$. The correlation between the NPH scale with the other scales was considered moderate to satisfactory for most items, ranging from - 
069 to 0.55 in Pearson correlation. The individual evaluation between scales on the items on urinary incontinence, dementia and gait were also satisfactory and stistically significant. The Portuguese version of the graduation scale for patients with NPH was successfully translated and validated for use in Brazilian patients of both genders, with satisfactory reliability and validity.

Descriptors: Hydrocephalus, normal pressure; Translation; Scales; Validation studeis; Questionnaires; Severity of illness index; Gait disorders, neurologic; Gait apraxia; Postural balance; Urinary incontinece/complications; Dementia/complications. 


\section{INTRODUÇÃO}

A hidrocefalia de pressão normal (HPN) é uma doença neurológica caracterizada por quadro progressivo de apraxia de marcha, alterações cognitivas e incontinência urinária (conhecida como síndrome de HakimAdams), associada à hidrocefalia comunicante (comprovada por tomografia de crânio ou ressonância magnética de encéfalo) e com a pressão do líquido cefalorraquidiano (LCR) dentro de valores normais (menos de vinte e quatro $\mathrm{cm}$ $\left.\mathrm{H}_{2} \mathrm{O}\right)^{1-7}$.

A HPN, primeiramente, foi reconhecida por Hakim e Adams et al. (1965), na década de 60 , os quais observaram um quadro de demência progressiva, alterações na marcha e dilatação do sistema ventricular ${ }^{1,8}$.

Dados epidemiológicos sobre incidência e prevalência de HPN são escassos $^{9}$. Acredita-se que corresponda a $5 \%$ de todas as demências e que a incidência seja de um em cada 100.000 pessoas $^{10}$. Estudos mostraram que, aproximadamente, $50 \%$ da incidência desta morbidade foram de origem idiopática e os demais 50\% HPN secundária ${ }^{11}$. Em contrapartida, Poca, Sahuquillo e Mataro $(2001)^{12}$ encontraram maior incidência de HPN secundária. Ainda de acordo com Krauss, Sebastian e Stuckrad-Barre (2008) ${ }^{11}$, a HPN acomete ambos os sexos, com preponderância maior, mas não significativa, entre as mulheres.

Segundo a literatura, a HPN manifesta-se, geralmente, a partir dos 55 anos, preponderantemente entre a sexta e sétima década de vida ${ }^{13}$. Conquanto a HPN secundária ocorra em indivíduos de todas as idades, vale ressaltar que a idiopática, preferencialmente, acomete indivíduos idosos.

Em relação ao quadro clínico, a HPN caracteriza-se, tipicamente, por um desenvolvimento gradual, de alterações na marcha, que progride insidiosamente com demência e incontinência urinária, sendo descrito como tríade clínica da síndrome de Hakim-Adams ${ }^{14,15}$. Essa tríade é considerada como marcador clínico da HPN, apesar da evolução ser oscilante, apresentando períodos de melhora e piora. Por sua vez, Poca, Sahuquillo e 
Mataro $(2001)^{12}$ relataram que os pacientes, geralmente, não apresentavam cefaleia ou outros sinais de hipertensão intracraniana (HIC).

Fernandez et al. $(2006)^{16}$ descreveram 36 pacientes com HPN e evidenciaram que 77,8\% apresentavam déficit cognitivo, 55,6\% alteração de marcha e $50 \%$ alteração urinária. Entretanto, somente $30,6 \%$ da população investigada apresentaram a tríade completa. Outros estudos descrevem a presença da tríade clínica em, aproximadamente, 50\% dos casos, todavia, apenas um ou a associação de dois dos sintomas devem ser igualmente considerados para investigação diagnóstica ${ }^{1-7}$.

O diagnóstico é iminentemente clínico/neurológico, corroborado por exames de neuroimagem ${ }^{5,17,18}$. O tratamento clássico e universalmente preconizado é a derivação ventriculoperitonial (DVP). Para os casos de HPN secundária (estenose de aqueduto, por exemplo), a terceiro-ventriculostomia endoscópica (TVE) é indicada ${ }^{19,20}$.

Estas manifestações ocasionadas pela HPN a tornam uma doença que compromete diretamente a qualidade de vida, principalmente a autonomia do paciente, mas vale advertir que, na maioria dos casos, é reversível com o tratamento adequado que associa 0 cirúrgico com a reabilitação multiprofissional $^{21}$. Ainda Blomsterwall et al. ${ }^{22}$ desvelaram que os elementos da tríade clínica podem comprometer a independência funcional do paciente e, frequentemente, são causadores de desajustes familiares e sociais.

Neste sentido, para que na avaliação inicial, no tratamento neurocirúrgico e multiprofissional, bem como nas avaliações subsequentes, seja possível classificar as reais limitações do paciente com critérios objetivos, a fim de determinar a evolução clínica e quantificar as mudanças observadas, é necessário um instrumento confiável e específico para mensurar os elementos tríade clínica ${ }^{23-26}$. 
2 OBJetivos 


\section{OBJETIVOS}

\subsection{Objetivo Geral}

Validar a "Grading Scale for Idiopathic Normal Pressure Hydrocephalus" desenvolvida na Língua Inglesa, a qual foi traduzida para a Língua Portuguesa como "Escala de Graduação do Paciente com Hidrocefalia de Pressão Normal".

\subsection{Objetivos específicos}

1) Traduzir e adaptar culturalmente para a Língua Portuguesa a "Grading Scale for Idiopathic Normal Pressure Hydrocephalus";

2) Investigar a correlação com a medida de independência funcional (MIF), índice de marcha dinâmica (DGI), teste de equilíbrio de Berg (BERG) e "Timed Up and Go" (TUG) nos pacientes portadores de HPN. 
3 REVISÃo dA LITERATURA 


\section{REVISÃO DA LITERATURA}

\subsection{Quadro clínico da HPN}

A sintomatologia da HPN está relacionada com o distúrbio da dinâmica liquórica ${ }^{27,28}$. Estudos de imagem revelaram dilatação ventricular sobre substância branca periventricular, sem atrofia cerebral significativa ${ }^{29}$. O volume liquórico circulante foi investigado por Tsunoda et al. ${ }^{30}$, em 2002, ao comparar medições em pacientes com HPN idiopática, HPN secundária, atrofia cerebral e indivíduos saudáveis, empregando ressonância magnética e programa processador de imagens. Os resultados encontrados foram apresentados no quadro 1, evidenciando importante diferença entre os grupos analisados ${ }^{30}$.

Quadro 1: Relação de volumes do líquido cefalorraquidiano intra e extraventricular conforme grupo de indivíduos analisados.

\begin{tabular}{|ccccc|}
\hline Grupos & Frequência & $\begin{array}{c}\text { Volume de LCR } \\
\text { intraventricular e } \\
\text { (desvio-padrão } \\
\text { [ml]) }\end{array}$ & $\begin{array}{c}\text { Volume total } \\
\text { de LCR e } \\
\text { (desvio- } \\
\text { padrão [ml]) }\end{array}$ & $\begin{array}{c}\text { Proporção entre } \\
\text { volume } \\
\text { ventricular; } \\
\text { volume LCR } \\
\text { (desvio-padrão } \\
\text { [\%]) }\end{array}$ \\
\hline $\begin{array}{c}\text { Hidrocefalia de } \\
\text { pressão normal } \\
\text { idiopática }\end{array}$ & 24 & $109,3(50,7)$ & $281,2(73,1)$ & $38,0(9,5)$ \\
$\begin{array}{c}\text { Hidrocefalia de } \\
\text { pressão normal } \\
\text { secundária }\end{array}$ & 17 & $71,3(18,2)$ & $196,9(46,5)$ & $37,3(8,9)$ \\
$\begin{array}{c}\text { Atrofia cerebral } \\
\text { Pessoa saudável }\end{array}$ & 21 & $64,9(25,3)$ & $284,4(54,5)$ & $22,8(7,7)$ \\
\hline
\end{tabular}

Fonte: Tsunoda et al., 2002.

A HPN apresenta um diagnóstico considerado difícil por ser uma doença sem sinais patognomônicos, uma vez que a tríade clássica distúrbio de marcha, incontinência urinária e declínio cognitivo pode ser comum ao 
envelhecimento, bem como advir de diferentes causas ${ }^{9}$. Todavia, diversas comorbidades prevalentes nesta faixa etária podem dificultar o diagnóstico.

Besch-azeddine et al. ${ }^{31}$ desvelaram que, aproximadamente, metade da população do estudo com HPN apresentaram critérios clínicos de outras doenças, como alterações cerebrovasculares ou Alzheimer ${ }^{31}$. Cerca de $40 \%$ a $75 \%$ dos pacientes com HPN tem beta-amiloide ou outros achados típicos de doença de Alzheimer, enquanto $60 \%$ possuem sinais de doença cerebrovascular que podem produzir achados clínicos similares à $\mathrm{HPN}^{32}$.

Ainda estudos anátomo-patológicos sugerem danos na substância branca periventricular nos lobos frontais, nos tálamos e gânglios da base provavelmente resultante de alterações pressóricas intermitentes que causam dilatação ventricular e transtorno metabólico ${ }^{33}$.

\subsubsection{Distúrbio da marcha}

Dentre os aspectos que despertam maior preocupação quanto às sequelas, o distúrbio da marcha emerge como predominante na clínica desses pacientes e, usualmente, precede de outros sintomas ${ }^{34}$.

$\mathrm{Na}$ literatura, esta marcha é descrita como apráxica ${ }^{35}$, a qual se manifesta pela incapacidade de executar uma sequência de movimentos, na ausência de sintomas motores e sensitivos ou incoordenação ${ }^{36}$. Essa dificuldade na locomoção e na manutenção do equilíbrio corporal ocasiona o risco de quedas. Dependendo do grau de comprometimento da marcha, os pacientes podem necessitar de dispositivos auxiliares de locomoção para deambularem, ou mesmo são incapazes de realizá-la ${ }^{29,37}$.

Comumente, os pacientes com HPN apresentam como característica a base de sustentação alargada, locomoção lenta, dificuldade para iniciar o movimento após estar em ortostatismo e após mudança de direção; acrescido de uma diminuição da movimentação dos braços e tendência de queda posterior. Perde-se a automatização da sequência do andar, que, diretamente, ocasiona um desequilíbrio constante durante a marcha aumentando a prevalência de queda ${ }^{22,38-40}$. Muitos pacientes relatam a sensação dos seus 
pés estarem presos ao solo durante o caminhar, sensação esta conhecida como marcha magnética ${ }^{29,41}$.

Consoante Zaaroor et al. ${ }^{42}$, em 1997, descreveram uma particularidade importante sobre a preservação funcional dos membros superiores, no qual avaliaram os quatro membros para localizar, por meio de um estudo eletrofisiológico, o preditor de sucesso do procedimento cirúrgico e excluiu seu comprometimento $^{42}$. Contudo, confirmaram que sobrevém uma dificuldade nos movimentos sincronizados dos membros superiores e inferiores durante a marcha por possuírem amplitude reduzida do passo ${ }^{40}$.

Entretanto, segundo Krauss, Sebastian e Stuckrad-Barre (2008) ${ }^{11}$, apesar do distúrbio de marcha ser um sintoma importante nessa população, não é patognomônico. Vale ressaltar que, na fase inicial da doença, pode ser impossível distingui-la da marcha senil e, posteriormente, a marcha instável pode ser confundida com outras doenças, como encefalopatia vascular subcortical (SVE).

\subsubsection{Alterações cognitivas}

A HPN é conhecida como um tipo de demência tratável, ou seja, pode haver reversão parcial ou completa do quadro clínico. Entretanto, apesar do aumento da prevalência dessa doença e crescentes publicações de diretrizes clínicas, ainda há controvérsias sobre diagnóstico e tratamento desses pacientes $^{43}$. Caracterizar detalhes da disfunção cognitiva é importante para diferenciá-la de outras demências neurodegenerativas ${ }^{44}$.

Segundo Iddon et al. (1999) ${ }^{45}$, a deteriorização cognitiva nesses pacientes não é totalmente reversível, pois, à medida que aumenta o tempo da doença, ocorre aumento da extensão dos danos cerebrais, afetando a substância branca subcortical e a substância cortical, o que favorece a alterações graves da função mental. Os mesmos autores observaram, em um subgrupo de HPN, melhora pós-cirúrgica em escalas globais de demência, todavia, não observaram melhora em testes psicológicos sensíveis a alterações do lobo frontal ${ }^{45}$. 
De acordo com Benejam et al. ${ }^{46}$, em 2008, os pacientes apresentaram quadros variados de deteriorização cognitiva, variando desde quadros leves, como dificuldade para recordar fatos recentes, déficit de atenção e concentração, até quadros graves, com lentidão no processamento das informações e desorientação têmporo-espacial. Igualmente, encontrou diminuição da espontaneidade, iniciativa, falta de interesse pelas atividades habituais e apatia ${ }^{46}$.

Muitos estudos da disfunção cognitiva nos pacientes com HPN sugerem ser do tipo subcortical ${ }^{47}$, focando-se na memória, atenção e função executiva como principais itens a serem afetados ${ }^{45,48}$. Saito et al. (2011) concluíram que as alterações pela HPN não se concentram apenas nos lobos frontais e nas funções executivas, mas também em regiões corticais posteriores com comprometimento da percepção visual e visuoespacial. Takeuchi et al. (2007), em seu estudo, evidenciaram que a inabilidade construtiva se deve à disfunção no lobo parietal, o que os diferencia dos pacientes com Alzheimer que apresentarem demência tipo frontotemporal, com parietal preservado ${ }^{48}$.

Atualmente, não há bateria de testes psicométricos específicos para essa população tanto para diagnóstico como para detecção de diferenças pré e póscirúrgicas. Entretanto, mesmo extensos, os testes neuropsicológicos não permitem a fácil diferenciação da HPN com outras demências subcorticais ${ }^{11}$.

\subsubsection{Incontinência urinária}

A incontinência urinária (IU) influencia diretamente a qualidade de vida dos pacientes, pois acarreta danos tanto na esfera psicológica e social, quanto na econômica e física. Há um consenso na literatura de que os próprios pacientes são seus melhores juízes na avaliação da interferência sobre a qualidade de vida ${ }^{49}$.

Assim, a incontinência urinária manifesta-se na maioria dos pacientes e raramente presenciamos a fecal. No estágio inicial da doença, é comum observar apenas urgência urinária e, nos estágios avançados, muitos pacientes tornam-se incontinentes. Assim, a IU não deve ser considerada apenas como 
consequência da demência, apesar do comprometimento cognitivo associado ao distúrbio de marcha favorecer a perda urinária, principalmente nos pacientes com urgência ${ }^{11}$.

Ahlberg et al. (1988) mostraram que a população de HPN investigada não apresentava comprometimento cognitivo ou de marcha que justificassem a incontinência, sendo estes achados clínicos independentes em cada paciente. Ainda durante exames urodinâmicos, os pacientes apresentaram hiperatividade da bexiga e instabilidade do detrusor, com significativa melhora do quadro clínico após "tap test" com remoção de cinquenta ml, ratificando que a HPN acomete regiões cerebrais que são importantes para o controle da micção ${ }^{50}$.

\subsection{Escalas de avaliação}

As escalas de avaliação são utilizadas na prática clínica por serem de fácil aplicação, baixo custo e dispensarem uso de equipamento especial ${ }^{21,25}$.

A realização adequada de tarefas do cotidiano demanda participação de funções motoras, sensitivas, cognitivas e psicológicas, assim, para avaliá-las no idoso, bem como analisar a interferência causada por diferentes doenças sobre sua independência funcional, deve-se realizar pesquisa clínica, testes físicos e aplicação de escalas, cujo conjunto denomina-se avaliação funcional. Itens como avaliação do equilíbrio, mobilidade, marcha, função cognitiva, capacidade de realizar atividades de vida diária (AVD), como andar, comer, vestir, ir ao toalete, e ter continência urinária e fecal devem ser avaliados de maneira clara e objetiva ${ }^{21,25}$.

A avaliação da marcha está relacionada com o equilíbrio corporal, visto que essa gera instabilidade do controle postural e cria um desafio adaptativo aos sistemas, tornando-se necessárias informações contínuas sobre posição e movimento das diferentes partes do corpo durante a locomoção. O controle da posição do corpo no espaço, como forma de promover estabilidade e orientação, é denominado de controle postural. A estabilidade postural é obtida por meio do repouso (equilíbrio estático), do movimento estável (equilíbrio dinâmico) ou pela recuperação do equilíbrio perdido durante determinada 
atividade. É fundamental para a manutenção do equilíbrio que o centro de massa corporal esteja projetado dentro dos limites da base de apoio, com integração das informações sensoriais com os sistemas neuromusculares ${ }^{41,36,37,51}$.

Estudos mostraram que as principais queixas dos pacientes portadores de HPN e de seus cuidadores são em relação à marcha. Deste modo, pode ser dada maior importância para a caracterização da marcha ao utilizar escalas de avaliação ${ }^{52}$. Todavia, para a avaliação completa de um paciente com HPN, os três componentes da tríade clínica devem ser considerados e, para este fim, podem-se utilizar associações de escalas que analisem os sintomas individualmente ${ }^{46}$.

\subsubsection{Medida de independência funcional}

A Medida de Independência Funcional (MIF) foi desenvolvida pela Academia Americana de Medicina Física e Reabilitação e pelo Congresso Americano de Medicina de Reabilitação na década de 1980, com intuito de mensurar o nível de ajuda que o portador de deficiência necessita para realizar tarefas motoras, como autocuidado, locomoção, vestuário, além de tarefas cognitivas. Foi traduzida e adaptada para a Língua Portuguesa em 2001, sendo válida, com boa equivalência cultural e reprodutividade ${ }^{53,54}$.

Composta por dezoito itens classificados em seis dimensões e duas subdivisões: motora e cognitiva. A MIF motora compreende autocuidados (alimentação, higiene pessoal, banho, vestir metade superior e inferior do corpo, uso do vaso sanitário), controle esfincteriano (continência urinária e fecal), mobilidade (vaso sanitário, banheira ou chuveiro), locomoção (andar e escadas). A MIF cognitiva abrange comunicação (compreensão e expressão) e cognição (interação social, resolução de problemas e memória) ${ }^{55}$ (Figura 1).

Cada item é pontuado até o nível sete, sendo um ponto para dependência total e sete independência total (Anexo A). A pontuação total da escala varia de dezoito a cento e vinte e seis, e, quanto menor, indica alta dependência. Independente do diagnóstico, esta escala avalia como o individuo está 
executando as atividades e não como executava em outras circunstâncias ou ambiente $^{53}$.

Sua aplicação não se faz por meio de autoaplicação, apresenta alta confiabilidade, desde que aplicado por terapeuta devidamente treinado e qualificado para utilização, com entrevista realizada pessoalmente com duração média de vinte a trinta minutos. Frequentemente, é necessária a presença do familiar ou cuidador para obtenção do perfil completo do paciente durante atividades de vida diária ${ }^{56}$.

$\mathrm{Na}$ literatura, diversos trabalhos utilizam a MIF para avaliação de pacientes com déficit cognitivo. Talmelli et al. (2010) investigaram a independência funcional dos idosos com doença de Alzheimer, por meio das escalas MIF e Miniexame do estado mental. Entre seus achados, evidenciaram que os déficits cognitivos influenciam no desempenho na realização das atividades da vida cotidiana, com uma média de pontuação de 107,7 para idosos sem déficit e 63,2 para os que o apresentavam ${ }^{55}$.

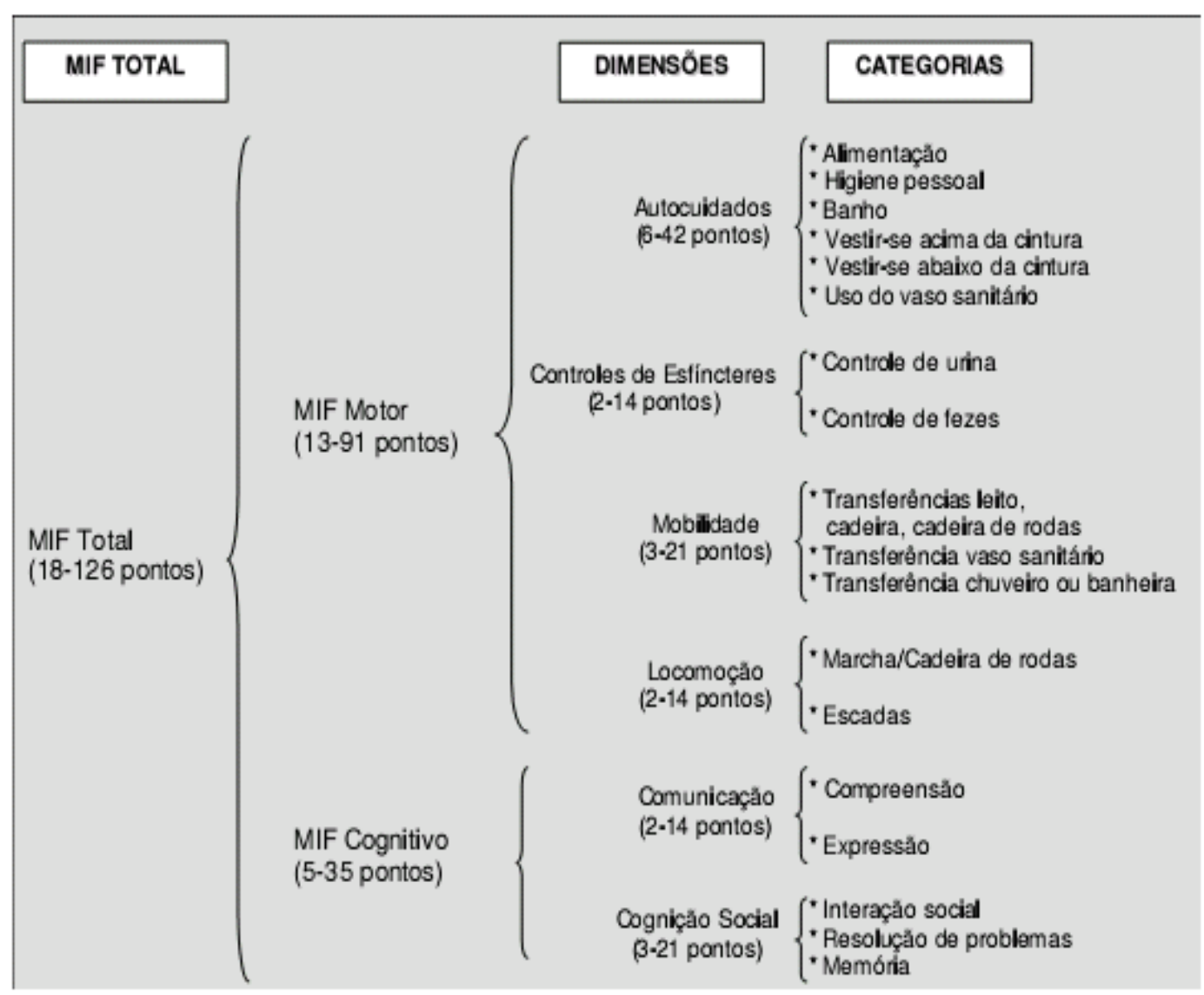

Figura 1 - Dimensões e pontuações da MIF. 


\subsubsection{Escala de equilíbrio de Berg}

Criada em 1992, é considerada escala de equilíbrio simples, fácil aplicação e largamente utilizada em pesquisa clínica, principalmente em idosos acima de 60 anos. Foi traduzida e adaptada para o Português em 2004 (Anexo B), demonstrando ser válida e confiável ${ }^{57}$.

Utilizada para determinar fatores de risco para perda, independência e quedas, descreve o equilíbrio funcional necessário para atividades de vida diária, como marcha, levantar de uma cadeira e movimentar os membros superiores enquanto mantêm-se na postura em ortostatismo. Igualmente, acompanha evolução das intervenções clínicas ${ }^{58}$.

Compreende quatorze domínios comuns à vida diária, cada item é pontuado de zero a quatro, com pontuação mínima e máxima variando entre zero e cinquenta e seis. O tempo é verificado a fim de manter determinada posição, alcance anterior dos membros superiores e o tempo total para completar uma tarefa ${ }^{57}$.

Para aplicação da escala, são necessários um relógio, uma régua, um banquinho e uma cadeira, e o tempo de execução é de, aproximadamente, 30 minutos, ainda pode ser realizada com pacientes vestidos, descalços e fazendo uso de óculos e/ou próteses auditivas de uso habitual ${ }^{57}$.

\subsection{3 Índice de marcha dinâmica}

Avalia a marcha dinâmica. Esta escala foi desenvolvida para identificar a probabilidade de queda em idosos. O teste é constituído das seguintes tarefas funcionais: caminhar em superfície plana; caminhar com mudanças na velocidade da marcha; caminhar com movimentos horizontais e verticais da cabeça; passar sobre e contornar obstáculos; girar sobre o próprio eixo corporal e subir/descer escadas ${ }^{59}$. Sugere-se, para realização da avaliação, demarcar o solo com fita adesiva no ponto de partida até atingir a marca de seis metros, e, nas distâncias de 1,80 metros e 3,60 metros, posicionar os cones $^{60}$. 
Apresenta vinte e quatro pontos de escore máximo, sendo que cada item recebe de zero a três pontos, sendo que o zero corresponde ao menor nível e o três o nível mais elevado da função (Anexo $\mathrm{C}$ ). A interpretação dos resultados indica que um valor menor ou igual a dezenove pontos é preditivo de quedas em idosos e que o indivíduo apresenta marcha segura apenas para valores superiores a vinte e dois pontos ${ }^{61}$.

\subsection{4 "Timed Up and Go"}

O teste "Get Up and Go" desenvolvido por Mathias et al. (1986) avalia, de forma padronizada, rápida e prática, a mobilidade. $O$ teste consiste em levantar de uma cadeira, caminhar três metros e retornar à posição inicial. Entretanto, há divergências entre observadores com relação à pontuação, pois essa era baseada apenas na percepção individual para classificá-los de acordo com desempenho na atividade proposta. Na década de 1990, foi desenvolvida versão modificada denominada "Timed Up \& Go" (TUG), na qual a avaliação da mobilidade teve como premissa os mesmos procedimentos acrescidos de uma marcação de tempo por um cronômetro. Muitos estudos evidenciam boa correlação do TUG com outras escalas, como escala de equilíbrio de Berg $(r=$ $-0.81)$ e escala Barthel $(r=-0.78)^{62}$.

As pontuações baseavam-se de acordo com tempo utilizado para realizar a tarefa, valores até dez segundos foram considerados dentro da normalidade para adultos independentes e sem risco de queda; sendo que, em idosos na mesma condição, são aceitáveis valores de até doze segundos. Valores entre onze a vinte segundos foram classificados como idoso frágil, independente parcial para atividades de vida diária ou com baixo risco de quedas. São classificados idosos com déficit de mobilidade e com risco de queda ${ }^{63}$ os idosos que obtêm pontuação entre vinte e um - trinta segundos; acima de trinta segundos, idoso com alta restrição de mobilidade ${ }^{62}$.

Esta escala é amplamente utilizada nas avaliações de mobilidade e de performance motora em idosos. Bischoff et al. (2003) investigaram a influência do ambiente sobre estes ao comparar a performance na realização do teste 
nos indivíduos que viviam na comunidade com os institucionalizados, o estudo demonstrou diferença na mobilidade ( $54 \%$ de variação, com os idosos da comunidade apresentando maior mobilidade funcional) ${ }^{63}$.

Feick et al. (2008) utilizaram o TUG, MIF, entre outras escalas para determinar as mudanças funcionais dos pacientes com HPN antes e após a punção lombar, com a terapia ocupacional e fisioterapia, e concluíram que a avaliação realizada foi sensível às mudanças após retirada do líquido cefalorraquidiano ${ }^{25}$.

\subsubsection{Escalas de graduação do paciente com HPN}

A primeira escala apresentada na literatura na Língua Inglesa foi a "Grading Scale for Idiopathic Normal Pressure Hydrocephalus" (Anexo D) desenvolvida no Japão. Sua vantagem prática é a facilidade e a rapidez de aplicação, além de avaliar a tríade clínica da HPN. Cada elemento (marcha, demência e incontinência urinária) é classificado em cinco pontos, que variam de zero a quatro, com pontuação máxima de doze e mínima de zero, sendo que, quanto maior o resultado, maior o grau de comprometimento do paciente $^{23}$.

Foi desenvolvida a partir de um estudo cooperativo entre 1996 e 1999 para determinar os critérios mais preditivos de diagnóstico para pacientes com HPN e que poderiam se beneficiar de procedimento cirúrgico. Para isso, questionários sobre essa doença foram enviados para quatorze membros do Comitê de Pesquisa Científica em Hidrocefalia, patrocinado pelo Ministério da Saúde e Bem-Estar do Japão. Posteriormente, uma análise retrospectiva das respostas foi realizada, em que incluía vários níveis de alteração de marcha, demência e incontinência urinária, sendo aplicada em cento e vinte pacientes ${ }^{23}$.

Outras escalas foram apresentadas na literatura, porém mantendo os princípios básicos da "Grading Scale for Idiopathic Normal Pressure Hydrocephalus". Kubo et al. (2008) desenvolveram a "Escala de Classificação de Hidrocefalia de Pressão Normal Idiopática" para classificar a tríade sintomática dos pacientes em diferentes gravidades. A pontuação foi baseada 
na observação dos terapeutas e entrevista com pacientes e seus cuidadores, cada domínio foi pontuado de zero a quatro, sendo que uma pontuação alta indica maior comprometimento.

O objetivo do estudo Kubo et al. (2008) foi avaliar a confiabilidade e validade da escala em trinta e oito pacientes com HPN idiopática. Para isso, foi comparada com Miniexame do Estado Mental (MMSE), o "Timed up and Go", escala de marcha e questionário internacional de incontinência (ICIQ-SF). Ainda para avaliar as mudanças da severidade da doença, foram aplicados, antes e após, os procedimentos coleta do líquido cefalorraquidiano e cirurgia. A confiabilidade interexaminadores da escala foi alta. O domínio cognitivo apresentou correlação estatisticamente significante com os escores do teste cognitivo, equivalente para os domínios marcha e incontinência urinária com os outros testes. Os pacientes que obtiveram melhora após coleta do líquor na escala de HPN também melhoraram nas demais escalas. Quatorze, dos trinta e oito pacientes, realizaram cirurgia quando houve associação significantes com as alterações cognitivas e da incontinência após retirada do líquor ${ }^{64}$.

Ishikawa et al. (2012) realizaram um estudo com cem pacientes de HPN com objetivo de determinar preditores de melhora clínica com indicação cirúrgica. Para isso, associou a aplicação do Minimental, "Timed up and go", Rankin e a Escala de Classificação de Hidrocefalia de Pressão Normal Idiopática, antes e após a retirada de trinta ml do líquido cefalorraquidiano por punção lombar, e antes, após três, seis e um ano de derivação ventrículoperitonial. Os achados deste estudo apresentaram uma resposta positiva, sendo que as melhores pontuações nas escalas estavam associadas à pressão de abertura do líquido cefalorraquidiano durante punção lombar maior ou igual a quinze $\mathrm{cm} \mathrm{H}_{2} \mathrm{O}^{65}$.

Ainda Hellstrom et al. (2012) apresentaram a escala de HPN que classifica a gravidade da doença e analisa os resultados do tratamento, desenvolvida por meio da avaliação de quatro domínios: marcha, equilíbrio, fatores neuropsicológicos e continência.

A marcha foi avaliada por meio de medições do número de passos e o tempo necessário para caminhar dez metros, o equilíbrio foi analisado por meio da observação do esforço do paciente em manter-se ereto de forma unipodal e 
bipodal. Para os fatores neuropsicológicos, foram utilizados testes como 0 "Grooved pegboard". Foi verificada continência pela percepção do paciente ou fonte mais confiável possível, visto que os pacientes com percepção reduzida podem negá-la, mesmo sendo incontinentes. Para este domínio, foi aplicada uma classificação ordinal, sendo que um foi considerado normal; dois, urgência, sem incontinência; três, rara incontinência, sem necessidade de absorventes/fralda; quatro, incontinência frequente, com absorvente/fralda; cinco, incontinência urinária; e seis, incontinência urinária e fecal ${ }^{66}$. 


\section{MÉTOdOS}




\section{MÉTODOS}

\subsection{Tradução e adaptação cultural}

A escala "Grading Scale for Idiopathic Normal Pressure Hydrocephalus" (Anexo D) foi traduzida para Língua Portuguesa de forma padronizada para garantir a qualidade e fidedignidade da versão original, de acordo com a metodologia de Guillemin et al. (1993) ${ }^{67}$, de acordo com Figura 2. A tradução para a Língua Portuguesa foi realizada separadamente por dois médicos brasileiros neurologistas bilíngues, experientes em validação de instrumentos, sendo que as duas traduções finais foram comparadas pelos tradutores, corrigindo as inconsistências, constituindo-se uma tradução consensual.

Posteriormente, foi realizada a retrotradução para Língua Inglesa por dois outros médicos neurologistas bilíngues, distintos dos anteriores e que desconheciam a escala original, com a finalidade de verificar se a tradução para a Língua Inglesa a partir da versão em Português resulta de um texto muito diferente da versão original em Inglês. Na ausência de diferença significativa, pode-se afirmar que a versão final traduzida foi equivalente à versão original.

Com relação à comparação das versões, esta foi realizada por um comitê multiprofissional, composto por médicos e fisioterapeutas do ambulatório não envolvidos no processo de tradução, definindo que a versão final em Português da escala fosse intitulada "Escala de Graduação do Paciente com Hidrocefalia de Pressão Normal" (Anexo E).

As funções do comitê foram revisar, modificar ou eliminar itens inadequados ou ambíguos, realizar adaptação cultural, enquanto assegura o conceito geral dos itens excluídos, visando garantir total compreensão da tradução.

Desta forma, seguiu-se a última fase de revisão para a Língua Portuguesa por uma profissional tradutora da área da saúde, a qual desconhecia a escala original e leiga quanto à patologia de Hidrocefalia de Pressão Normal. A 
finalidade desta etapa foi detectar qualquer inadequação da versão final com adaptação cultural, sendo solicitada uma avaliação de equivalência de cada item entre versão original e final. Assim, os itens com baixo nível de equivalência poderiam ser novamente revisados pela comissão multiprofissional. O resultado foi pré-testado em um estudo-piloto.

A tradução foi realizada no Ambulatório do Grupo de Hidrodinâmica Cerebral da Divisão de Neurocirurgia Funcional do Instituto de Psiquiatria do Hospital das Clínicas em 2008, e, desde então, tem sido sistematicamente utilizada neste ambulatório em todos os pacientes com HPN para facilitar graduação dos diferentes quadros apresentados e acompanhamento da evolução da doença ao longo do tempo. Entretanto, não havia um protocolo para validação dessa versão para Língua Portuguesa a fim de verificar sua confiabilidade.

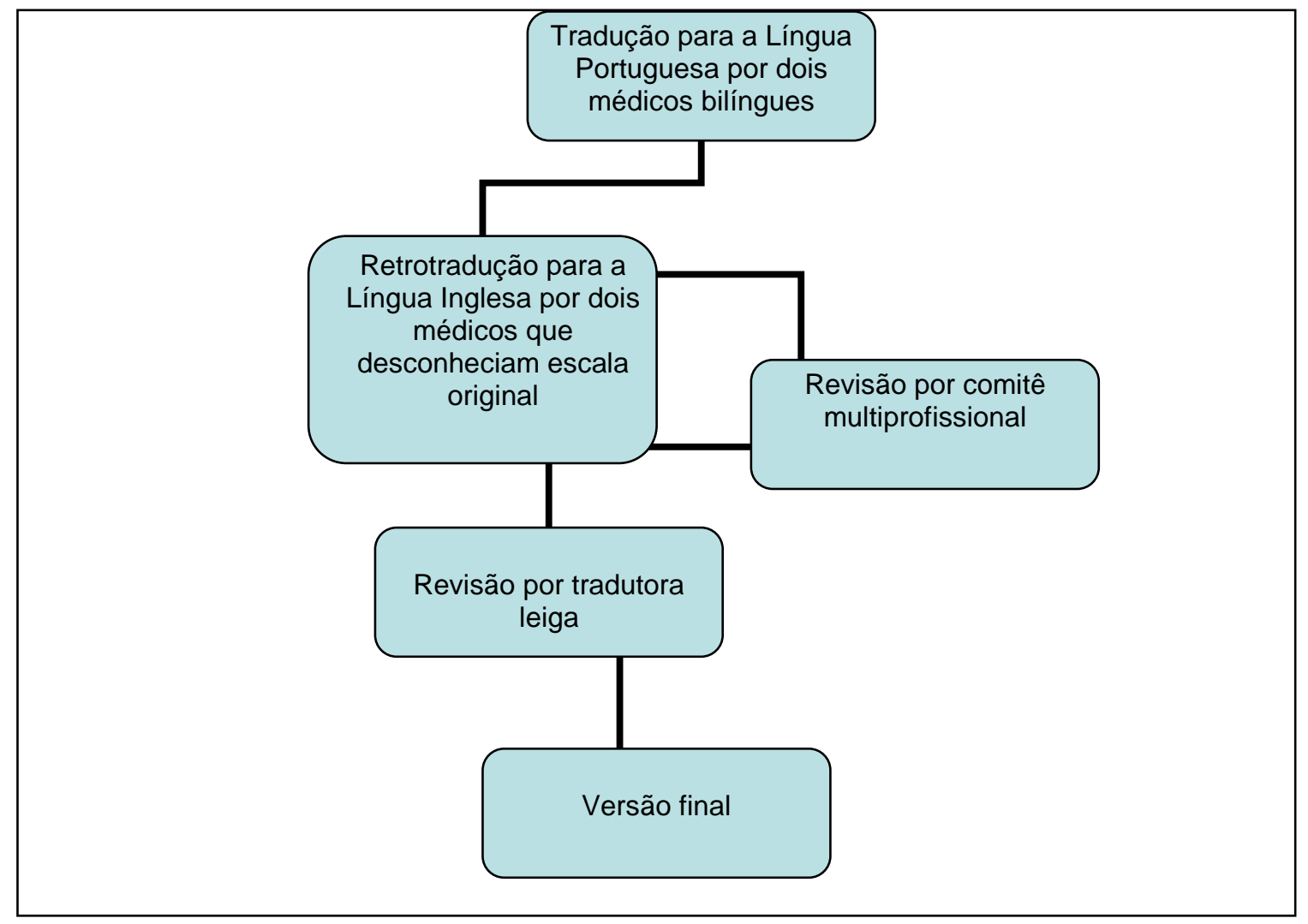

Figura 2 - Sequência da tradução da escala. 


\subsection{Validação da escala}

Os pacientes foram individualmente submetidos à avaliação em dois momentos diferentes, com intervalo de uma semana. Os dados foram coletados por uma fisioterapeuta $(A)$ e, posteriormente, outra fisioterapeuta $(B)$ pontuou, sequencialmente, o mesmo paciente. Deste modo, foi possível confrontar os resultados e evitar erro interobservador (Tabela 1).

No primeiro dia, foi coletado o protocolo inicial, contendo dados pessoais e aplicação das escalas MIF, BERG, DGI, TUG e escala de graduação da HPN pela fisioterapeuta (A) para que estes resultados pudessem ser comparados de modo a testar a confiabilidade do novo instrumento.

Tabela 1 - Categorias e escores da escala de graduação do paciente com HPN, coletados durante as avaliações.

\begin{tabular}{c|c|c|c|c|c}
\hline \multirow{2}{*}{ CATEGORIAS } & \multicolumn{5}{|c}{ ESCORES } \\
\cline { 2 - 6 } & 0 & 1 & 2 & 3 & 4 \\
\hline Distúrbio de marcha & & & & & \\
\hline Demência & & & & & \\
\hline Incontinência urinária & & & & & \\
\hline
\end{tabular}

No mesmo dia, a segunda fisioterapeuta (B) aplicou a escala de graduação da HPN a fim de verificar a reprodutividade interobservadores. Após uma semana, o paciente foi submetido à nova avaliação da escala de graduação da HPN pela fisioterapeuta inicial (A) a fim de verificar a reprodutividade intraobservador (teste/reteste), desde que não houvesse significativa diferença do quadro clínico, Figura 3.

O presente estudo foi aprovado pela Comissão de Ética para Análise de Projetos de Pesquisa (CAPpesq) do Hospital das Clínicas da Faculdade de Medicina da Universidade de São Paulo (HCFMUSP) (Anexo F) e, teve como critério não intervir na rotina hospitalar, sem realizar intervenções terapêuticas, somente aplicação das escalas e dos testes físicos, por meio de uma sequência de avaliação: 


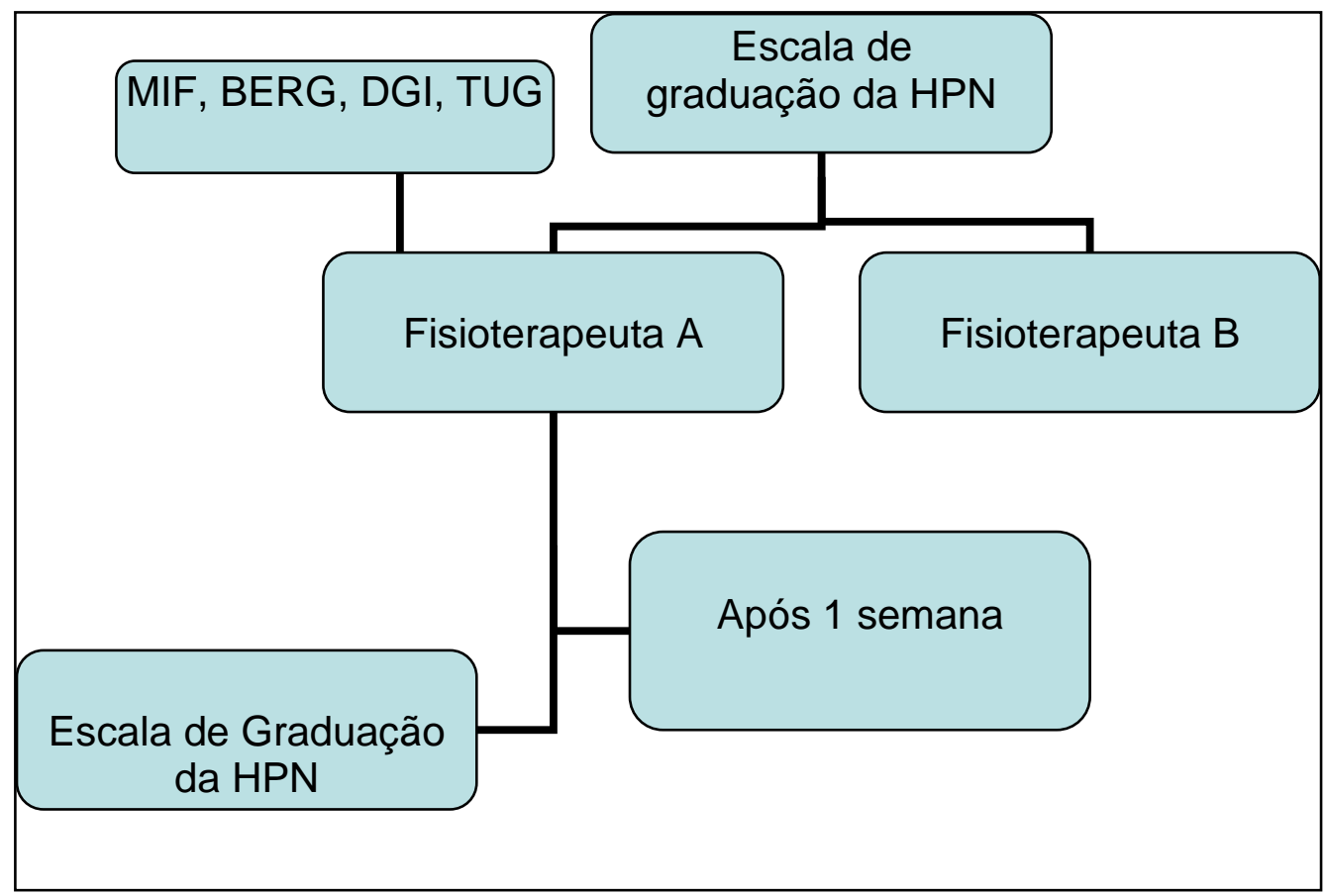

Figura 3 - Sequência da avaliação funcional.

\subsubsection{Medida de Independência Funcional - MIF}

Escala selecionada, pois, no meio clínico, é considerada completa, devido a englobar tanto o componente motor quanto o cognitivo, possui ampla aceitação na literatura nacional e internacional, bem como é relevante ser validada em diferentes países ${ }^{53}$.

Atende a critérios de confiabilidade, validade, precisão, praticidade e facilidade ${ }^{68}$.

É composta por dezoito itens relacionados a autocuidados, controle esfincteriano, mobilidade, locomoção, comunicação e cognição. A pontuação final deve variar de dezoito a cento e vinte e seis; e, quanto menor, indica maior dependência (Tabela 2), independente do diagnóstico, esta escala avalia como o indivíduo está executando as atividades atualmente e não como executava em outras circunstâncias ou ambiente. É um instrumento confiável desde aplicado por terapeuta devidamente qualificado para utilizá-lo ${ }^{53,54}$. 
Tabela 2 - Categorias e escores da escala medida de independência funcional, coletados durante as avaliações.

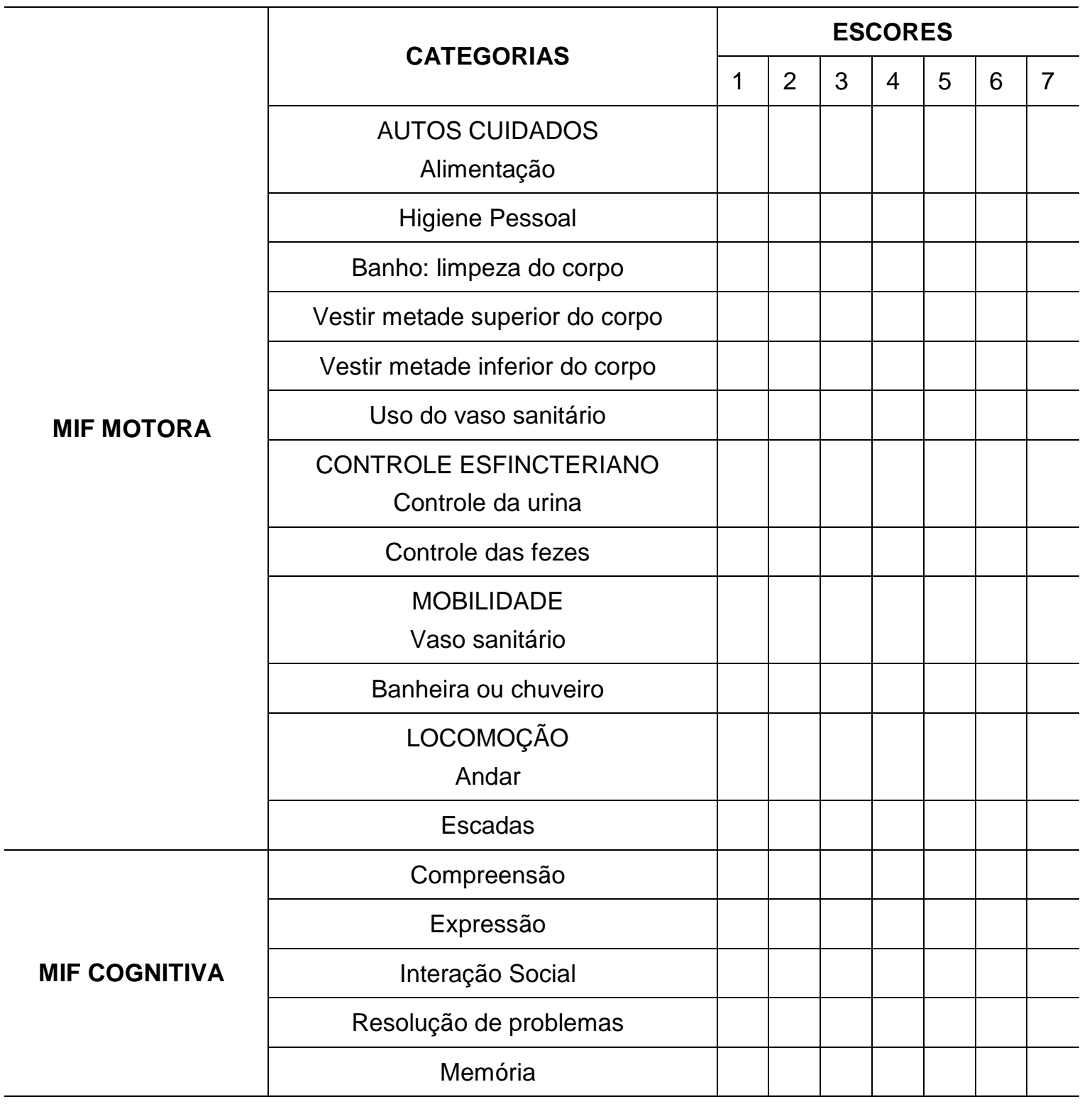

\subsubsection{Teste de Equilíbrio de Berg (Berg)}

Este teste avalia o equilíbrio em diferentes tarefas funcionais, constituído por uma escala de quatorze tarefas comuns que envolvem o equilíbrio estático e dinâmico, tais como alcançar, girar, transferir-se, permanecer em pé e levantar-se. A realização das tarefas é avaliada por meio da observação e a pontuação varia de zero a quatro, totalizando um máximo de cinquenta e seis 
pontos (Tabela 3). Estudos demonstraram forte correlação com outros testes de mobilidade, como "Timed Up and Go"57-70.

Tabela 3 - Categorias e escores do teste de equilíbrio de Berg, coletados durante as avaliações.

\begin{tabular}{l|l|l|l|l|l}
\hline \multicolumn{1}{c|}{ CATEGORIAS } & \multicolumn{5}{c}{ ESCORES } \\
\cline { 2 - 6 } & 0 & 1 & 2 & 3 & 4 \\
\hline Posição sentada para posição em pé & & & & & \\
\hline Permanecer em pé sem apoio & & & & & \\
\hline Permanecer sentado sem apoio nas costas & & & & & \\
\hline Posição em pé para posição sentada & & & & & \\
\hline Transferências: cadeira-cadeira & & & & & \\
\hline Permanecer em pé com os olhos fechados & & & & & \\
\hline Permanecer em pé sem apoio, pés juntos & & & & & \\
\hline Alcance anterior & & & & & \\
\hline Virar-se e olhar para trás, sobre os ombros & & & & & \\
\hline Girar 360 & & & \\
\hline Posicionar pés alternados no degrau & & & & & \\
\hline Permanecer em pé, sem apoio, um dos pés à frente & & & & & \\
\hline Permanecer em pé sobre uma das pernas & & & & & \\
\hline
\end{tabular}

\subsection{3 Índice de marcha dinâmica}

Avalia a capacidade do paciente de modificar a marcha em resposta às mudanças nas demandas de determinadas tarefas funcionais. É dividido em oito itens: marcha em superfície plana, mudanças de velocidade, movimentos horizontais e verticais de cabeça, passar sobre e ao redor de obstáculos, giro sobre próprio eixo corporal, subir e descer escadas.

A pontuação é dada pelo nível de disfunção, com pontuação máxima de vinte e quatro para pacientes independentes funcionalmente e mínima de zero para comprometimento grave e incapaz de realizar as atividades propostas (Tabela 4). Pode ser utilizado como preditor de quedas, quando a pontuação é 
igual ou inferior 19, e considerada marcha segura para pontuações acima de $22^{60,61}$.

Tabela 4 - Categorias e escores do teste Índice de marcha dinâmica, coletados durante as avaliações.

\begin{tabular}{l|l|l|l|l}
\hline \multirow{2}{*}{ CATEGORIAS } & \multicolumn{4}{c}{ ESCORES } \\
\cline { 2 - 5 } & 0 & 1 & 2 & 3 \\
\hline Marcha em superfície plana & & & & \\
\hline Mudança de velocidade & & & & \\
\hline Movimentos horizontais da cabeça & & & & \\
\hline Movimentos verticais da cabeça & & & & \\
\hline Passar sobre obstáculo & & & & \\
\hline Passar ao redor de obstáculo & & & & \\
\hline Girar $360^{\circ}$ & & & & \\
\hline Subir e descer escada & & & & \\
\hline
\end{tabular}

\subsection{4 “Timed Up and Go” (TUG)}

Segundo Loth et al. (2004), o teste denominado "Timed Up and Go" (TUG) representa um instrumento de avaliação rápida da mobilidade funcional em pessoas idosas ${ }^{71}$. O paciente inicia o teste sentado em uma cadeira padrão de 46 centímetros, com encosto e apoio para braço. Ao comando verbal "vá", deveria levantar da cadeira, caminhar de forma confortável e segura em linha reta por três metros, dar meia-volta, retornar e sentar na posição inicial. 0 paciente não deve receber auxílio físico do terapeuta e faz uso do tempo como unidade de medida do resultado (Tabela 5$)^{62}$.

Tabela 5 - Categorias e escores do TUG.

\begin{tabular}{l|l}
\hline \multicolumn{1}{c|}{ CATEGORIAS } & \multicolumn{1}{c}{ ESCORES } \\
\hline 10 segundos & Normal \\
\hline $11-20$ segundos & Idoso frágil \\
\hline $21-30$ segundos & Idoso com déficit de mobilidade \\
\hline Acima de 30 segundos & Idoso com alta restrição de mobilidade \\
\hline
\end{tabular}




\subsection{Tipo de estudo}

O delineamento metodológico proposto caracterizou-se por uma pesquisa de validação de escala por meio da comparação e utilização com outras escalas.

Foi realizado com pacientes com HPN (idiopática ou secundária) provenientes do Ambulatório de Hidrodinâmica Cerebral da Divisão de Neurocirurgia Funcional - FMUSP, no período de julho de 2010 a março de 2012.

Todos pacientes assinaram o Termo de Consentimento Livre e Esclarecido (TCLE) (Anexo G) e atenderam às especificações dos critérios de inclusão e exclusão.

\subsection{Casuística}

Foram estudados pacientes com diagnóstico médico de HPN, de ambos os gêneros, com idade acima de dezoito anos. Os pacientes foram recrutados por meio de uma amostra consecutiva de conveniência, de acordo com os critérios de inclusão e exclusão.

\subsection{Critérios de inclusão e exclusão}

Foram incluídos pacientes com diagnóstico de HPN idiopática ou secundária, acima de dezoito anos, ambos os gêneros que consentiram sua participação por meio do Termo de Consentimento Livre e Esclarecido.

Foram excluídos do estudo pacientes com alteração do nível de consciência (menos que quatorze pontos na Escala de Coma de Glasgow); portadores doenças sistêmicas clinicamente descompensadas que interferiram na coleta da avaliação funcional. 


\section{Resultados}




\section{RESULTADOS}

\subsection{Tradução e adaptação cultural}

Após o método de tradução, obteve-se a versão para Língua Portuguesa da escala "Grading Scale for Idiopathic Normal Pressure Hydrocephalus", Tabela 6.

Tabela 6 - Escala de graduação de HPN.

\begin{tabular}{l|l}
\hline \multicolumn{1}{c|}{ ITENS } & \\
\hline Distúrbio de Marcha & Ausente \\
\hline 1 & Marcha instável, mas independente \\
\hline 2 & Anda com 1 apoio \\
\hline 3 & Anda com 2 apoios ou andador \\
\hline 4 & Não é possível andar \\
\hline Demência & \\
\hline 0 & Ausente \\
\hline 1 & Sem demência aparente, mas apático \\
\hline 2 & Socialmente dependente, mas independente na residência \\
\hline 3 & Parcialmente dependente na residência \\
\hline 4 & Totalmente dependente \\
\hline Incontinência Urinaria & \\
\hline 0 & Ausente \\
\hline 1 & Ausente, mas com polaciúria ou urgência miccional \\
\hline 2 & Às vezes, apenas à noite \\
\hline 3 & Às vezes, mesmo durante o dia \\
\hline 4 & Frequente \\
\hline
\end{tabular}

Para verificar validação da nova versão, seguiu-se aplicação sistemática do protocolo nos pacientes do Ambulatório de Hidrodinâmica. 
As características gerais desses pacientes encontram-se na Tabela 7.

Tabela 7 - Características dos 121 pacientes segundo faixa etária e gênero, que foram avaliados no Ambulatório do Grupo de Hidrodinâmica Cerebral do IPQ-HCFMUSP, no período de junho de 2010 a fevereiro de 2012.

\begin{tabular}{c|c|c|c}
\hline Categoria & Frequência & $\%$ & Desvio-padrão \\
\hline Gênero & & & \\
Masculino & 73 & 60,33 & \\
Feminino & 48 & 39,67 & \\
\hline Faixa etária (anos) & & & \\
Abaixo 60 & 17 & 14,05 & 9,1 \\
61 a 70 & 33 & 27,27 & 2,9 \\
71 a 80 & 46 & 38,01 & 2,8 \\
Acima 81 & 25 & 20,67 & 2,8 \\
\hline
\end{tabular}

A Tabela 7 mostra maior frequência dos pacientes do gênero masculino. A variação geral da idade foi entre 35 e 92 anos, com maior concentração de pacientes entre 71 e 80 anos. A idade média de 71,09 anos com $d p=2,8$ anos.

Quanto aos domínios, a Tabela 8 evidencia maior frequência de comprometimento da marcha $(96,69 \%)$.

Tabela 8 - Frequências absolutas e relativas das características relacionadas aos domínios da amostra.

\begin{tabular}{c|c|c}
\hline Domínio & $\mathbf{n}$ & $\%$ \\
\hline Marcha & 117 & 96,69 \\
Demência & 104 & 85,95 \\
Incontinência & 96 & 79,33 \\
\hline
\end{tabular}

\subsection{Confiabilidade e reprodutividade}

Para avaliação da confiabilidade e reprodutividade, a escala foi aplicada pelo mesmo avaliador com intervalo de uma semana (análise intra-avaliador) e diferentes avaliadores no dia (análise interavaliador).

Os resultados foram analisados pela medida de concordância Kappa. 
O teste estatístico Kappa é baseado no número de respostas concordantes, ou seja, no número de casos com mesmo resultado e determina o grau de concordância além do esperado pelo acaso. Para determinar se o resultado obtido é satisfatório, utiliza-se a interpretação demonstrada na Tabela 9.

Tabela 9 - Interpretação da concordância segundo valores da medida de Kappa

\begin{tabular}{c|c}
\hline Valores de Kappa & Interpretação da concordância \\
\hline Abaixo 0 & Ausente \\
\hline 0 a 0.19 & Pobre \\
\hline 0.20 a 0.39 & Baixa \\
\hline 0.40 a 0.59 & Moderada \\
\hline 0.60 a 0.79 & Satisfatória \\
\hline 0.80 a 1.0 & Excelente \\
\hline
\end{tabular}

\subsubsection{Concordância intra-avaliador}

A concordância intra-avaliador foi, inicialmente, aplicada para 0 item marcha ao confrontar como categoria a pontuação de zero a quatro, como demonstra a Tabela 10. Para concordância excelente, foram obtidas as pontuações quatro e um.

Tabela 10 - Valores de Kappa para as diferentes pontuações da escala.

\begin{tabular}{|c|c|c|c|c|c|}
\hline & 0 & 1 & 2 & 3 & 4 \\
\hline Kappa por pontuação & 0,698 & 0,824 & 0,729 & 0,696 & 0,95 \\
\hline $\begin{array}{l}\text { P-valor do Kappa da } \\
\text { pontuação }\end{array}$ & $<0,001$ & $<0,001$ & $<0,001$ & $<0,001$ & $<0,001$ \\
\hline $\begin{array}{c}\text { Intervalo de } 95 \% \text { de } \\
\text { confiança do Kappa das } \\
\text { pontuações }\end{array}$ & $\begin{array}{l}\text { sup:0,868 } \\
\text { inf:0,528 }\end{array}$ & $\begin{array}{c}\text { sup: } 1,0 \\
\text { inf:0,648 }\end{array}$ & $\begin{array}{l}\text { sup:0,905 } \\
\text { inf:0,552 }\end{array}$ & $\begin{array}{l}\text { sup:0,872 } \\
\text { inf: } 0,52\end{array}$ & $\begin{array}{l}\text { sup: } 1,0 \\
\text { inf:0,772 }\end{array}$ \\
\hline $\begin{array}{c}\text { Intervalo de } 95 \% \text { de } \\
\text { confiança do Kappa das } \\
\text { pontuações }\end{array}$ & $\begin{array}{l}\text { sup: } 1,0 \\
\text { inf:0,685 }\end{array}$ & $\begin{array}{l}\text { sup:0,916 } \\
\text { inf:0,563 }\end{array}$ & $\begin{array}{l}\text { sup:0,909 } \\
\text { inf:0,556 }\end{array}$ & $\begin{array}{l}\text { sup: } 1,0 \\
\text { inf: } 0,717\end{array}$ & $\begin{array}{l}\text { sup: } 1,0 \\
\text { inf:0,789 }\end{array}$ \\
\hline
\end{tabular}


O valor Kappa geral do item marcha obteve-se concordância excelente (Tabela 11).

Tabela 11 - Kappa geral do item marcha (intra-avaliador).

\begin{tabular}{c|c}
\hline Kappa geral & $\mathbf{0 , 8}$ \\
\hline P-valor geral & $<0,001$ \\
\hline Intervalo de $95 \%$ de confiança do & sup:0,899 \\
Kappa & inf: 0,702 \\
\hline
\end{tabular}

Para análise do item demência, ao comparar como categoria a pontuação zero a quatro (Tabela 12), concordância excelente para todas as pontuações.

Tabela 12 - Valores de Kappa para as diferentes pontuações da escala.

\begin{tabular}{c|c|c|c|c|c}
\hline & $\mathbf{0}$ & $\mathbf{1}$ & $\mathbf{2}$ & $\mathbf{3}$ & $\mathbf{4}$ \\
\hline Kappa por pontuação & 0,928 & 0,875 & 0,834 & 0,895 & 0,979 \\
\hline $\begin{array}{c}\text { P-valor do Kappa da } \\
\text { pontuação }\end{array}$ & $<0,001$ & $<0,001$ & $<0,001$ & $<0,001$ & $<0,001$ \\
\hline $\begin{array}{c}\text { Intervalo de 95\% de } \\
\text { confiança do Kappa das } \\
\text { pontuações }\end{array}$ & $\begin{array}{c}\text { sup: } 1,0 \\
\text { inf:0,75 }\end{array}$ & $\begin{array}{c}\text { sup:1,0 } \\
\text { inf:0,698 }\end{array}$ & $\begin{array}{c}\text { sup: } 1,0 \\
\text { inf:0,657 }\end{array}$ & $\begin{array}{c}\text { sup: } 1,0 \\
\text { inf:0,717 }\end{array}$ & $\begin{array}{c}\text { sup:1,0 } \\
\text { inf:0,801 }\end{array}$ \\
\hline
\end{tabular}

O valor encontrado do Kappa geral, de 0,90, indica concordância excelente entre as medidas intra-avaliador (Tabela 13).

Tabela 13 - Kappa geral do item demência (intra-avaliador).

\begin{tabular}{c|c}
\hline Kappa geral & $\mathbf{0 , 9 0 5}$ \\
\hline P-valor geral & $<0,001$ \\
\hline Intervalo de $95 \%$ de confiança do & sup:0,997 \\
Kappa & inf: 0,812 \\
\hline
\end{tabular}

Para análise do item incontinência urinária, obteve-se concordância excelente para pontuações zero, três e quatro. Concordância satisfatória para um e dois (Tabela 14). 
Tabela 14 - Valores de Kappa para as diferentes pontuações da escala.

\begin{tabular}{c|c|c|c|c|c}
\hline & $\mathbf{0}$ & $\mathbf{1}$ & $\mathbf{2}$ & $\mathbf{3}$ & $\mathbf{4}$ \\
\hline Kappa por pontuação & 0,864 & 0,74 & 0,733 & 0,895 & 0,967 \\
\hline $\begin{array}{c}\text { P-valor do Kappa da } \\
\text { pontuação }\end{array}$ & $<0,001$ & $<0,001$ & $<0,001$ & $<0,001$ & $<0,001$ \\
\hline
\end{tabular}

Concordância excelente para medida Kappa geral do item incontinência urinária (Tabela 15).

Tabela 15 - Kappa geral do item incontinência (intra-avaliador).

\begin{tabular}{c|c}
\hline Kappa geral & $\mathbf{0 , 8 7 1}$ \\
\hline P-valor geral & $<0,001$ \\
\hline $\begin{array}{c}\text { Intervalo de 95\% de confiança do } \\
\text { Kappa }\end{array}$ & sup:0,971 \\
inf: 0,772 \\
\hline
\end{tabular}

\subsubsection{Concordância interavaliador}

Posteriormente, foi realizada análise da validação do método por meio da Concordância interavaliador, por meio do Kappa.

Para a marcha (Tabela 16), obteve-se como resultado concordância excelente para as pontuações um, dois, três e quatro.

O valor Kappa geral do item marcha interobservador, com concordância de 0,91, excelente, pode ser observado na Tabela 17.

Tabela 16 - Valores de Kappa para as diferentes pontuações.

\begin{tabular}{c|c|c|c|c|c}
\hline & $\mathbf{0}$ & $\mathbf{1}$ & $\mathbf{2}$ & $\mathbf{3}$ & $\mathbf{4}$ \\
\hline Kappa por pontuação & 0,698 & 0,895 & 0,928 & 0,932 & 0,974 \\
\hline $\begin{array}{c}\text { P-valor do Kappa da } \\
\text { pontuação }\end{array}$ & $<0,001$ & $<0,001$ & $<0,001$ & $<0,001$ & $<0,001$ \\
\hline $\begin{array}{c}\text { Intervalo de 95\% de } \\
\text { confiança do Kappa das } \\
\text { pontuações }\end{array}$ & $\begin{array}{c}\text { sup:0,868 } \\
\text { inf:0,528 }\end{array}$ & $\begin{array}{c}\text { sup: } 1,0 \\
\text { inf:0,718 }\end{array}$ & $\begin{array}{c}\text { sup: } 1,0 \\
\text { inf:0,749 }\end{array}$ & $\begin{array}{c}\text { sup: } 1,0 \\
\text { inf:0,753 }\end{array}$ & $\begin{array}{c}\text { sup:1,0 } \\
\text { inf:0,796 }\end{array}$ \\
\hline
\end{tabular}


Tabela 17 - Kappa geral do item marcha.

\begin{tabular}{c|c}
\hline Kappa geral & $\mathbf{0 , 9 1 1}$ \\
\hline P-valor geral & $<0,001$ \\
\hline Intervalo de $95 \%$ de confiança do \\
Kappa & sup: 1,0 \\
& inf: 0,813 \\
\hline
\end{tabular}

Concordância excelente para todas as pontuações do item demência na análise interavaliador (Tabela 18).

Tabela 18 - Valores de Kappa para as diferentes pontuações.

\begin{tabular}{c|c|c|c|c|c}
\hline & $\mathbf{0}$ & $\mathbf{1}$ & $\mathbf{2}$ & $\mathbf{3}$ & $\mathbf{4}$ \\
\hline Kappa por pontuação & 0,833 & 0,823 & 0,868 & 0,825 & 0,938 \\
\hline $\begin{array}{c}\text { P-valor do Kappa da } \\
\text { pontuação }\end{array}$ & $<0,001$ & $<0,001$ & $<0,001$ & $<0,001$ & $<0,001$ \\
\hline $\begin{array}{c}\text { Intervalo de 95\% de } \\
\text { confiança do Kappa das } \\
\text { pontuações }\end{array}$ & $\begin{array}{c}\text { sup: } 1,0 \\
\text { inf:0,656 }\end{array}$ & $\begin{array}{c}\text { sup: } 1,0 \\
\text { inf:0,645 }\end{array}$ & $\begin{array}{c}\text { sup: } 1,0 \\
\text { inf:0,69 }\end{array}$ & $\begin{array}{c}\text { sup: } 1,0 \\
\text { inf:0,647 }\end{array}$ & $\begin{array}{c}\text { sup:1,0 } \\
\text { inf:0,76 }\end{array}$ \\
\hline
\end{tabular}

O valor Kappa geral do item demência com concordância excelente (Tabela 19).

Tabela 19 - Kappa geral do item demência (interavaliador).

\begin{tabular}{c|c}
\hline Kappa geral & $\mathbf{0 , 8 6 3}$ \\
\hline P-valor geral & $<0,001$ \\
\hline Intervalo de 95\% de confiança do & sup:0,955 \\
Kappa & inf: 0,772 \\
\hline
\end{tabular}

Para análise interavaliador do item incontinência urinária ao comparar como categorias as pontuações de zero a quatro (Tabela 20), houve concordância excelente para 0,3 e 4 e moderada para 2.

Tabela 20 - Valores de Kappa para as diferentes pontuações.

\begin{tabular}{c|c|c|c|c|c}
\hline & $\mathbf{0}$ & $\mathbf{1}$ & $\mathbf{2}$ & $\mathbf{3}$ & $\mathbf{4}$ \\
\hline Kappa por pontuação & 0,946 & 0,766 & 0,52 & 0,848 & 0,983 \\
\hline $\begin{array}{c}\text { P-valor do Kappa da } \\
\text { pontuação }\end{array}$ & $<0,001$ & $<0,001$ & $<0,001$ & $<0,001$ & $<0,001$ \\
\hline $\begin{array}{c}\text { Intervalo de 95\% de } \\
\text { confiança do Kappa das } \\
\text { pontuações }\end{array}$ & $\begin{array}{c}\text { sup: } 1,0 \\
\text { inf:0,768 }\end{array}$ & $\begin{array}{c}\text { sup:0,944 } \\
\text { inf:0,589 }\end{array}$ & $\begin{array}{c}\text { sup:0,698 } \\
\text { inf:0,342 }\end{array}$ & $\begin{array}{c}\text { sup: } 1,0 \\
\text { inf:0,672 }\end{array}$ & $\begin{array}{c}\text { sup:1,0 } \\
\text { inf:0,805 }\end{array}$ \\
\hline
\end{tabular}


Para o valor Kappa geral do item incontinência urinária (interavaliador), obteve-se concordância excelente, 0,87 (Tabela 21).

Tabela 21 - Kappa geral do item incontinência urinária.

\begin{tabular}{c|c}
\hline Kappa geral & $\mathbf{0 , 8 7 1}$ \\
\hline P-valor geral & $<0,001$ \\
\hline Intervalo de $95 \%$ de confiança do & sup:0,971 \\
Kappa & inf: 0,771 \\
\hline
\end{tabular}

A análise do método foi bastante concordante tanto inter como intraavaliador, classificando-o como válido.

\subsection{Comparações dos valores da escala de graduação HPN com demais escalas selecionadas}

A comparação da escala de graduação HPN com as demais escalas foram realizadas por meio do coeficiente de correlação de Pearson (c), teste ANOVA e comparações múltiplas de Turkey.

A correlação de Pearson determina o grau de associação entre duas variáveis de mensuração numérica. A proximidade do valor um (positivo ou negativo) indica maior grau de associação entre as informações, de acordo com a classificação (Tabela 22).

Tabela 22 - Correlações segundo valor de Pearson.

\begin{tabular}{c|c}
\hline Valor de Pearson & Correlação \\
\hline$|c|<0,40$ & Fraca \\
\hline $0,40<|c|<0,70$ & Moderada \\
\hline $0,70<|c|<0,90$ & Boa \\
\hline$|c|>0,90$ & Ótima \\
\hline
\end{tabular}

O teste de ANOVA (Análise de Variância) é utilizado para comparar informações numéricas com intuito de identificar se, em médias, os grupos são diferentes. Já o teste de Tukey resulta em intervalos menores que qualquer 
outro método de comparação múltipla de uma etapa. A estratégia de Tukey consiste em definir a menor diferença significativa.

\subsubsection{Comparação dos valores HPN com a independência funcional (MIF)}

Foram utilizados para comparação entre os valores da escala HPN e a escala MIF total a correlação de Pearson (Tabela 23) e o teste de Análise de Variância (ANOVA), demonstrada na Tabela 24. Para resultado significante, utilizaram, posteriormente, comparações múltiplas de Tukey (Tabela 25).

Tabela 23 - Comparação dos valores totais das escalas HPN e MIF, por meio da Correlação de Pearson.

\begin{tabular}{l|c|c}
\hline \multirow{4}{*}{ MIF Total } & Correlação de Pearson & HPN - Total 1 \\
\cline { 2 - 3 } & Sig.(p) &,$- 842\left(^{* *}\right)$ \\
\cline { 2 - 3 } & $\mathbf{N}$ & 0 \\
\hline
\end{tabular}

${ }^{* *}$ Correlação significante a 0,01 .

A correlação pode ser considerada satisfatória.

Tabela 24- ANOVA, com tabela de médias e desvios-padrão.

\begin{tabular}{c|c|c|c|c}
\hline \multirow{2}{*}{} & \multicolumn{3}{|c|}{ MIF } & \\
\cline { 2 - 5 } & $<40$ & $\mathbf{4 0 - 7 9}$ & $\mathbf{8 0 +}$ & Total \\
\hline Média & 11,2 & 7,61 & 4,42 & 66,95 \\
\hline $\begin{array}{c}\text { desvio- } \\
\text { padrão }\end{array}$ & 1,323 & 2,382 & 2,331 & 27,468 \\
\hline $\mathbf{N}$ & 25 & 36 & 60 & 211 \\
\hline
\end{tabular}

ANOVA $(p)=<0,001^{*}$ 
Tabela 25- Comparações múltiplas de Tukey.

\begin{tabular}{c|c|c}
\hline Comparações & $(\mathbf{p})$ & Resultado \\
\hline$(<40) \times(40-79)$ & $<0,001^{*}$ & Diferentes \\
\hline$(<40) \times(80+)$ & $<0,001^{*}$ & Diferentes \\
\hline$(40-79) \times(80+)$ & $<0,001^{*}$ & Diferentes \\
\hline
\end{tabular}

Obteve-se a hierarquia segundo valores de HPN: $(<40)>(40-79)>(80+)$, (Gráfico 1).

Gráfico 1 - Demonstra pontuações da MIF, segundo HPN.

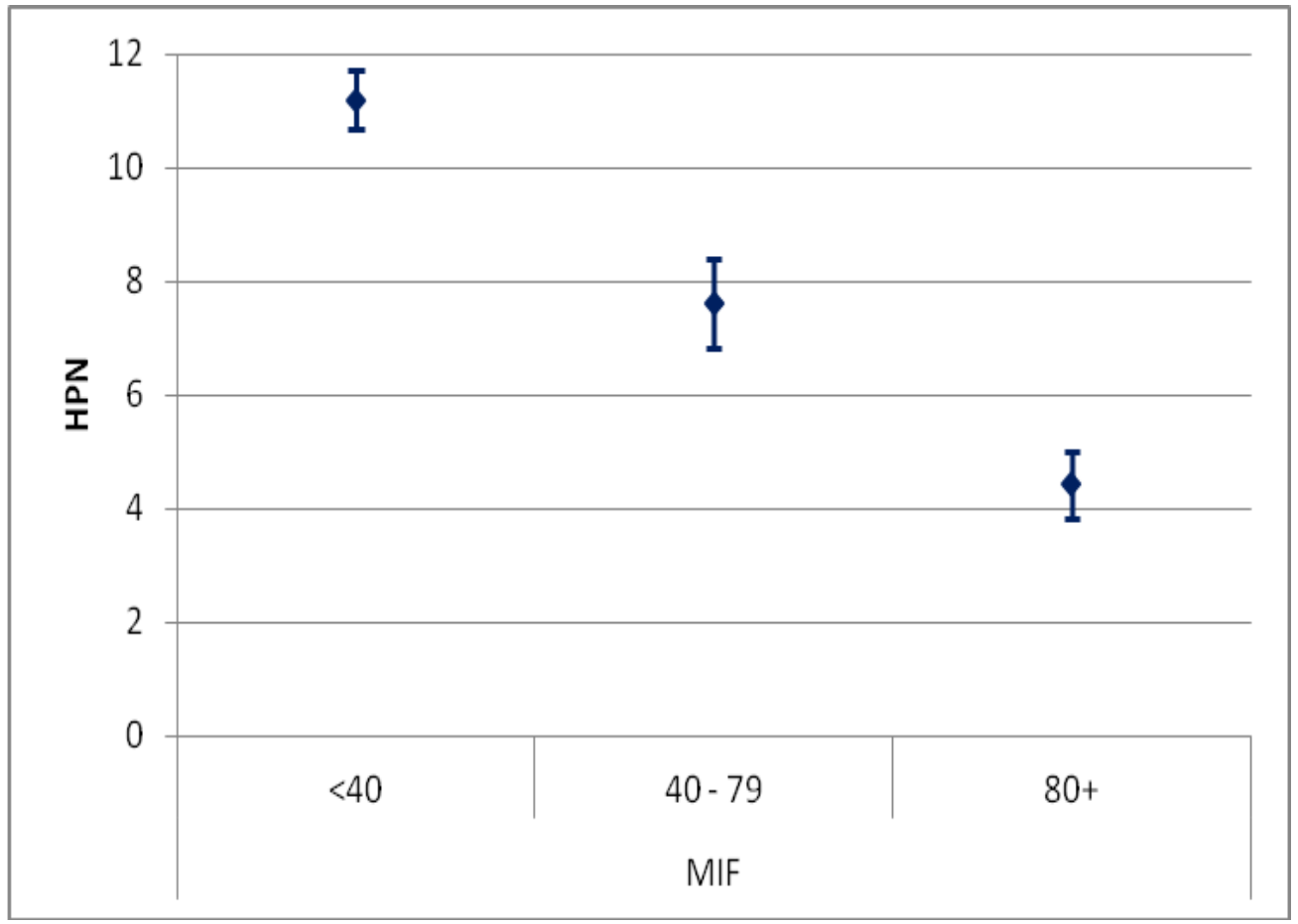

Intervalo de confiança para a média: média $\pm 1,96^{*}$ desvio-padrão $/ \sqrt{ }(n-1)$

\subsubsection{Comparação dos valores HPN com a escala de BERG}

A comparação entre os valores de HPN e a escala BERG apresenta correlação de Pearson (Tabela 26) e teste de Análise de Variância (ANOVA), demonstrada na Tabela 27. Posteriormente, comparações múltiplas de Tukey (Tabela 28). 
Tabela 26 - Comparação dos valores das escalas HPN e BERG, por meio da correlação de Pearson.

\begin{tabular}{c|c|c}
\hline & & HPN - Total 1a \\
\hline \multirow{3}{*}{ BERG(0-56) } & Correlação de Pearson &,$\left.- 803^{* *}\right)$ \\
\cline { 2 - 3 } & Sig.(p) & 0 \\
\cline { 2 - 3 } & $\mathbf{N}$ & 121 \\
\hline
\end{tabular}

${ }^{* *}$ Correlação significante a 0,01.

A correlação pode ser considerada satisfatória.

Tabela 27 - ANOVA, com tabela de médias e desvios-padrão.

\begin{tabular}{l|c|c|c|c}
\hline \multirow{2}{*}{} & \multicolumn{3}{|c|}{ BERG } & \\
\cline { 2 - 5 } & $\mathbf{< 6}$ & $\mathbf{3 6 - 4 5}$ & $\mathbf{4 6 +}$ & Total \\
\hline Média & $\mathbf{8 , 5 6}$ & 4,57 & 3,17 & 66,95 \\
\hline desvio-padrão & 2,925 & 1,942 & 2,036 & 27,468 \\
\hline
\end{tabular}

ANOVA $(p)=<0,001^{*}$.

Tabela 28 - Comparações múltiplas de Tukey.

\begin{tabular}{c|c|c}
\hline Comparações & $(\mathbf{p})$ & Resultado \\
\hline$(<36) \times(36-45)$ & $<0,001^{*}$ & Diferentes \\
\hline$(<36) \times(46+)$ & $<0,001^{*}$ & Diferentes \\
\hline$(36-45) \times(46+)$ & 0,171 & Iguais \\
\hline
\end{tabular}

Obteve-se a hierarquia segundo valores de HPN: $(<36)>(36-45)=(46+)$, (Gráfico 2). 
Gráfico 2 - Demonstra pontuações da BERG, segundo HPN.

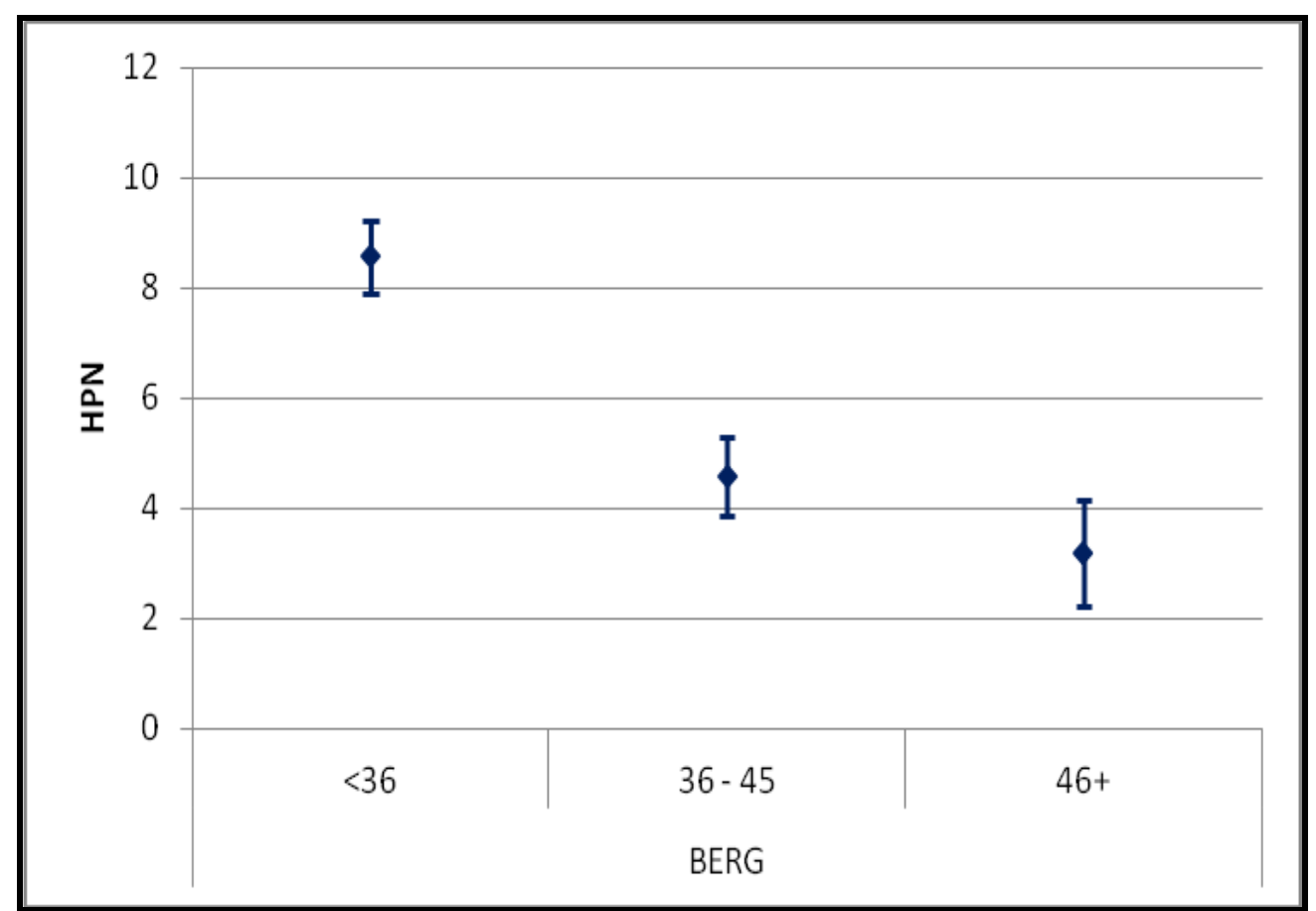

Intervalo de confiança para a média: média $\pm 1,96$ * desvio-padrão $/ \sqrt{ }(n-1)$

\subsubsection{Comparação dos valores HPN com a marcha dinâmica (DGI)}

Quanto à comparação entre os valores de HPN e a escala DGI, apresenta a correlação de Pearson (Tabela 29) e teste t-independente (student), demonstrada na Tabela 30.

Tabela 29 - Comparação dos valores das escalas HPN e DGI, por meio da Correlação de Pearson.

\begin{tabular}{c|c|c}
\hline \multirow{4}{*}{ DGI(0-24) } & Correlação de Pearson & HPN - Total 1 \\
\cline { 2 - 3 } & Sig.(p) & 0 \\
\cline { 2 - 3 } & $\mathbf{N}$ & 121 \\
\hline
\end{tabular}

${ }^{\star *}$ Correlação significante a 0,01 . 
Tabela 30 - Teste t-independente, com tabela de médias e desvios-padrão.

\begin{tabular}{l|c|c|c}
\hline \multirow{2}{*}{} & \multicolumn{2}{|c|}{ DGI } & \\
\cline { 2 - 4 } & $<=\mathbf{1 9}$ & $\mathbf{2 0 +}$ & Total \\
\hline Média & 6,98 & 3,75 & 66,95 \\
\hline desvio-padrão & 3,399 & 2,315 & 27,468 \\
\hline $\mathbf{N}$ & 113 & 8 & 211 \\
\hline
\end{tabular}

Teste $t(p)=0,009^{*}$

Obteve-se a hierarquia segundo valores de HPN: $(<=19)>(20+)$ (Gráfico 3).

Gráfico 3 - Demonstra pontuações da DGI, segundo HPN.

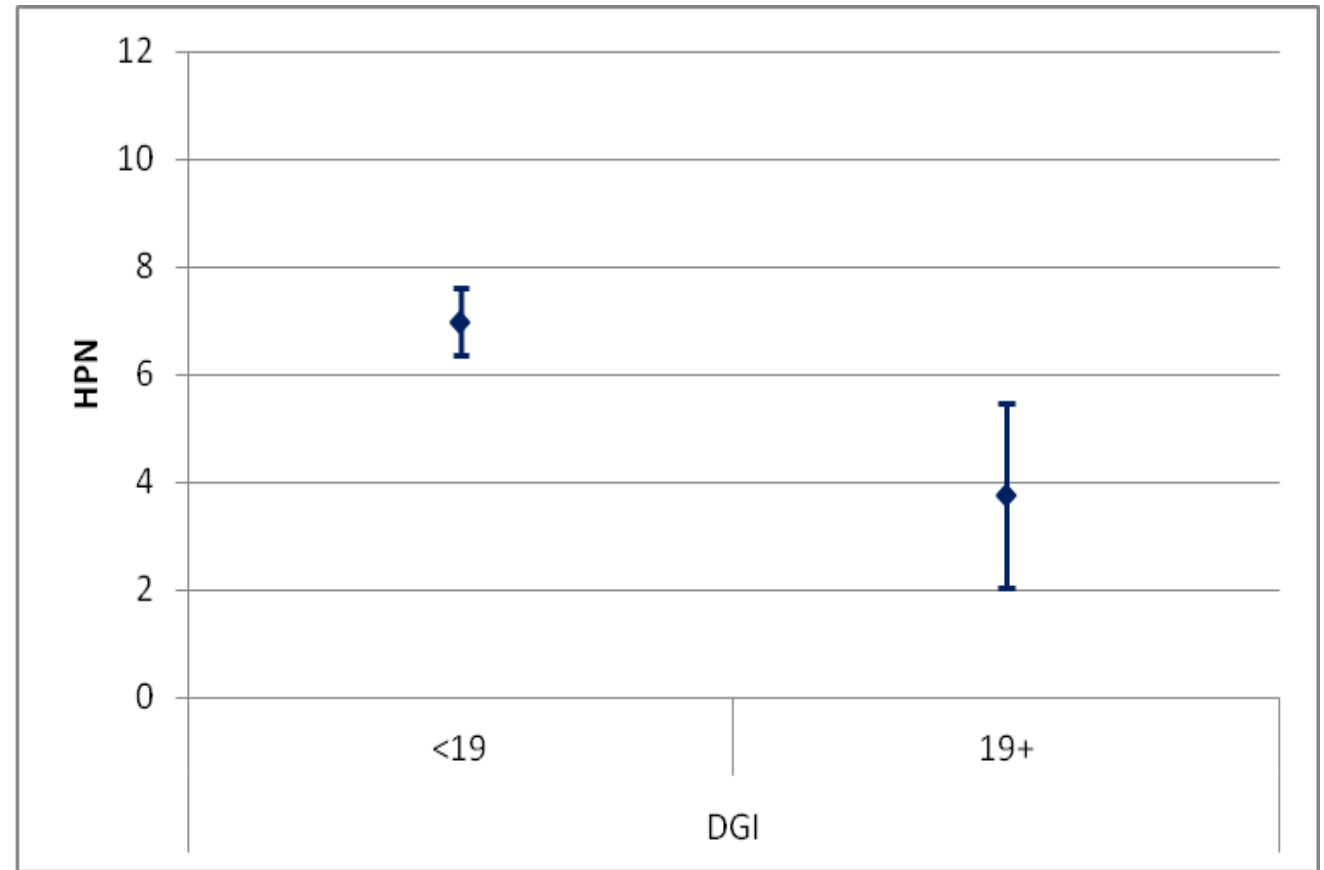

Intervalo de confiança para a média: média $\pm 1,96$ * desvio-padrão $/ \sqrt{ }(n-1)$

\subsubsection{Comparação dos valores HPN com "Timed Up and Go" (TUG)}

A correlação entre HPN e a escala TUG (Tabela 31) foi realizada por meio do teste de Análise de Variância (ANOVA), demonstrada na Tabela 32, e, se significante, posteriormente, por meio das comparações múltiplas de Tukey (Tabela 33). 
Tabela 31 - Comparação dos valores da escala HPN e TUG, por meio da Correlação de Pearson

\begin{tabular}{c|l|c}
\hline \multirow{2}{*}{ TUG_cat } & Correlação de Pearson & HPN - Total 1a \\
\cline { 2 - 3 } & Sig.(p) & $0,557\left(^{* *}\right)$ \\
\cline { 2 - 3 } & $\mathbf{N}$ & 121 \\
\hline
\end{tabular}

${ }^{* *}$ Correlação significante a 0,01 .

A correlação é satisfatória.

Tabela 32 - ANOVA, com tabela de médias e desvios-padrão.

\begin{tabular}{l|c|c|c|c|c}
\hline \multirow{2}{*}{} & \multicolumn{4}{|c|}{ TUG } & \\
\cline { 2 - 6 } & até 12 & $\mathbf{1 3}$ a 19 & $\mathbf{2 0}$ a 29 & $\mathbf{3 0 +}$ & Total \\
\hline Média & 3,14 & 4,82 & 6,06 & 8,32 & 66,95 \\
\hline desvio-padrão & 2,107 & 2,281 & 2,772 & 3,183 & 27,468 \\
\hline $\mathbf{N}$ & 14 & 22 & 17 & 68 & 211 \\
\hline
\end{tabular}

$\operatorname{ANOVA}(p)=<0,001^{*}$

Tabela 33 - Comparações múltiplas de Tukey.

\begin{tabular}{c|c|c}
\hline Comparações & $(\mathbf{p})$ & Resultado \\
\hline$($ até 12$) \times(13$ a 19$)$ & 0,327 & Iguais \\
\hline$($ até 12$) \times(20$ a 29$)$ & $0,029^{*}$ & Diferentes \\
\hline$($ até 12$) \times(30+)$ & $<0,001^{*}$ & Diferentes \\
\hline$(13$ a 19) $\times(20$ a 29 $)$ & 0,543 & Iguais \\
\hline$(13$ a 19) $\times(30+)$ & $<0,001^{*}$ & Diferentes \\
\hline$(20$ a 29$) \times(30+)$ & $0,023^{*}$ & Diferentes \\
\hline
\end{tabular}

Obteve-se a hierarquia segundo valores de HPN: (até 12$)=(13-19)=(20$ $29)<(30+)$. (até 12) < (20-29) (Gráfico 4). 
Gráfico 4 - Demonstra valores do TUG, segundo HPN.

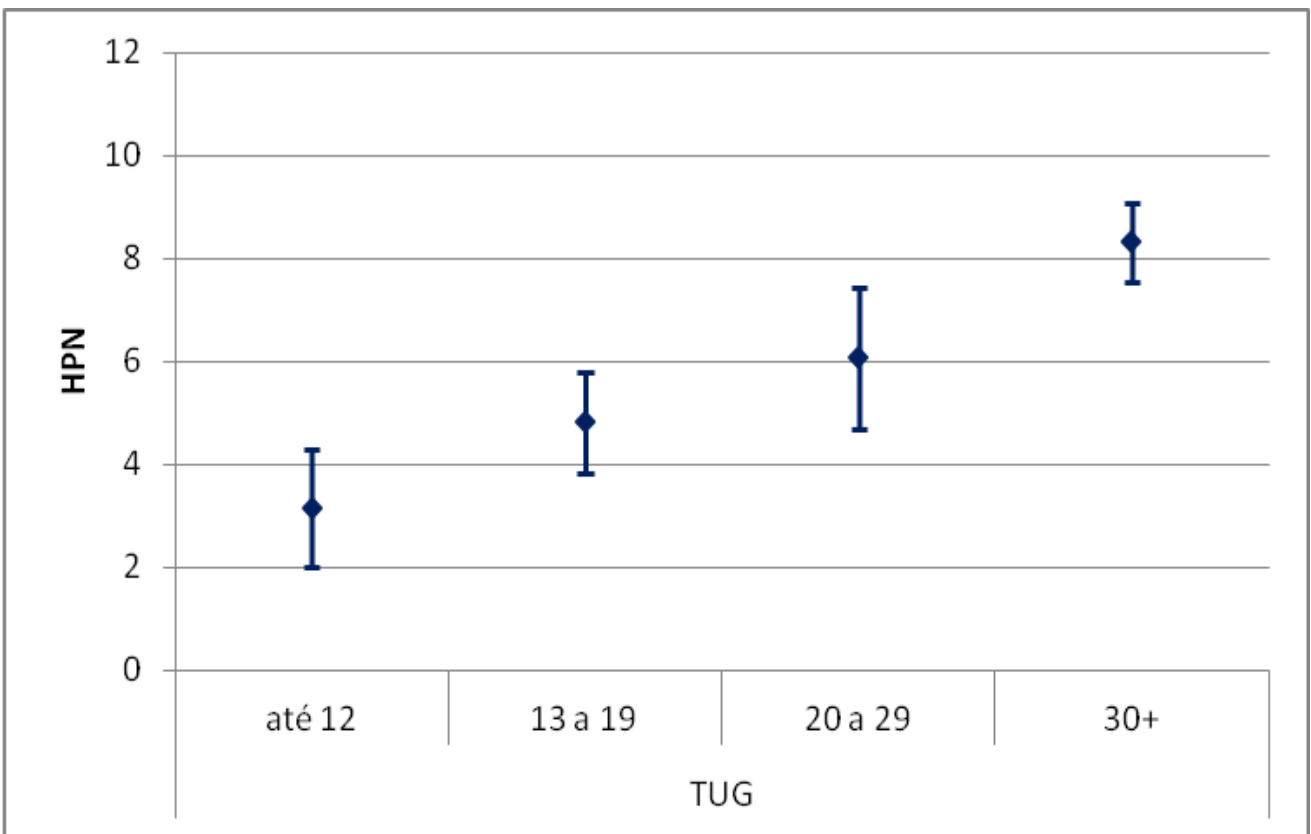

Intervalo de confiança para a média: média $\pm 1,96$ * desvio-padrão $/ \sqrt{ }(n-1)$

\subsection{Correlações entre escalas sobre item incontinência urinária}

A análise da comparação dos valores do item " $G$ " da MIF com o item incontinência urinária da HPN foi realizada por meio da correlação de Pearson (Tabela 34).

Tabela 34 - Comparação apenas dos valores dos itens relacionados à continência das escalas HPN e MIF, por meio da Correlação de Pearson.

\begin{tabular}{c|l|c}
\hline \multirow{2}{*}{$\mathbf{G}$} & Correlação de Pearson & Inc Urinária1 \\
\cline { 2 - 3 } & Sig.(p) &,$- 884\left(^{* *}\right)$ \\
\cline { 2 - 3 } & $\mathbf{N}$ & 0 \\
\hline
\end{tabular}

A correlação é satisfatória. 
Tabela 35 - Comparação descritiva segundo os valores dos itens de continência das escalas HPN e MIF.

\begin{tabular}{c|c|c|c|c|c|c|c}
\hline \multirow{2}{*}{} & \multicolumn{5}{|c|}{ Inc Urinaria1 } & \multirow{2}{*}{ Total } \\
\cline { 2 - 8 } & & $\mathbf{0}$ & $\mathbf{1}$ & $\mathbf{2}$ & $\mathbf{3}$ & $\mathbf{4}$ & \\
\hline \multirow{4}{*}{$\mathbf{G}$} & $\mathbf{1}$ & 0 & 0 & 1 & 2 & 39 & 42 \\
\cline { 2 - 8 } & $\mathbf{2}$ & 0 & 1 & 5 & 2 & 15 & 23 \\
\cline { 2 - 8 } & $\mathbf{3}$ & 0 & 1 & 0 & 5 & 1 & 7 \\
\cline { 2 - 8 } & $\mathbf{4}$ & 1 & 2 & 1 & 1 & 1 & 6 \\
\cline { 2 - 8 } & $\mathbf{5}$ & 1 & 4 & 2 & 7 & 0 & 14 \\
\cline { 2 - 8 } & $\mathbf{6}$ & 4 & 6 & 0 & 0 & 0 & 10 \\
\cline { 2 - 8 } & $\mathbf{7}$ & 17 & 2 & 0 & 0 & 0 & 19 \\
\hline \multicolumn{2}{c|}{ Total } & 23 & 16 & 9 & 17 & 56 & 121 \\
\hline
\end{tabular}

Note que maiores valores do item "G" da MIF correspondem a valores mais baixos em incontinência urinária da HPN e vice-versa (Tabela 35).

\subsection{Correlações entre escalas sobre item cognição}

Foi utilizada para determinar a comparação dos valores apenas da MIF cognitiva ( $N, O, P, Q, R)$ com o item demência da HPN para a correlação de Pearson (Tabela 36).

Tabela 36 - Comparação apenas dos valores dos itens relacionados à cognição da MIF com item demência da HPN, por meio da Correlação de Pearson.

\begin{tabular}{c|c|c}
\hline \multirow{4}{*}{ NOPQR } & $\begin{array}{c}\text { Correlação de } \\
\text { Pearson }\end{array}$ & Dem1 \\
\cline { 2 - 3 } & Sig.(p) &,$- 741\left(^{* *}\right)$ \\
\cline { 2 - 3 } & $\mathbf{N}$ & 0 \\
\hline
\end{tabular}

${ }^{* *}$ Correlação significante a 0,01 .

A correlação é considerada boa. 
Tabela 37 - Comparação descritiva segundo os valores dos itens relacionados à cognição da HPN e MIF.

\begin{tabular}{|c|c|c|c|c|c|c|c|}
\hline & & \multicolumn{5}{|c|}{ Dem1 } & \multirow{2}{*}{ Total } \\
\hline & & 0 & 1 & 2 & 3 & 4 & \\
\hline \multirow{29}{*}{ NOPQR } & 5 & 0 & 0 & 0 & 0 & 7 & 7 \\
\hline & 6 & 0 & 0 & 0 & 2 & 4 & 6 \\
\hline & 7 & 0 & 0 & 2 & 1 & 1 & 4 \\
\hline & 8 & 0 & 0 & 0 & 1 & 2 & 3 \\
\hline & 10 & 0 & 0 & 0 & 0 & 3 & 3 \\
\hline & 11 & 0 & 0 & 0 & 0 & 1 & 1 \\
\hline & 12 & 0 & 0 & 0 & 0 & 4 & 4 \\
\hline & 13 & 0 & 0 & 0 & 0 & 2 & 2 \\
\hline & 14 & 0 & 0 & 0 & 0 & 1 & 1 \\
\hline & 15 & 0 & 0 & 0 & 0 & 1 & 1 \\
\hline & 16 & 0 & 1 & 0 & 2 & 1 & 4 \\
\hline & 18 & 0 & 1 & 1 & 0 & 0 & 2 \\
\hline & 19 & 0 & 0 & 2 & 0 & 0 & 2 \\
\hline & 20 & 0 & 2 & 1 & 0 & 0 & 3 \\
\hline & 21 & 0 & 1 & 2 & 0 & 2 & 5 \\
\hline & 22 & 0 & 0 & 1 & 0 & 1 & 2 \\
\hline & 23 & 0 & 1 & 0 & 3 & 0 & 4 \\
\hline & 24 & 0 & 2 & 2 & 1 & 1 & 6 \\
\hline & 25 & 0 & 1 & 0 & 2 & 0 & 3 \\
\hline & 26 & 0 & 0 & 1 & 1 & 2 & 4 \\
\hline & 27 & 0 & 2 & 0 & 0 & 0 & 2 \\
\hline & 28 & 1 & 4 & 1 & 0 & 0 & 6 \\
\hline & 29 & 2 & 2 & 5 & 1 & 0 & 10 \\
\hline & 30 & 0 & 3 & 1 & 0 & 0 & 4 \\
\hline & 31 & 2 & 4 & 0 & 1 & 0 & 7 \\
\hline & 32 & 1 & 1 & 2 & 0 & 0 & 4 \\
\hline & 33 & 3 & 4 & 0 & 1 & 0 & 8 \\
\hline & 34 & 3 & 1 & 3 & 1 & 0 & 8 \\
\hline & 35 & 4 & 1 & 0 & 0 & 0 & 5 \\
\hline \multicolumn{2}{|c|}{ Total } & 16 & 31 & 24 & 17 & 33 & 121 \\
\hline
\end{tabular}

Encontraram-se valores altos na MIF cognitiva que são correspondentes aos valores mais baixos em demência da HPN e vice-versa, visto que as escalas são inversamente proporcionais (Tabela 37). 


\subsection{Correlações entre escalas sobre item marcha}

A correlação de Pearson foi utilizada para verificar a comparação dos valores somados dos itens locomoção da MIF (L, M) com o item marcha da HPN (Tabela 38).

Tabela 38 - Comparação apenas dos valores dos itens relacionados à marcha da MIF com item marcha da HPN, por meio da Correlação de Pearson.

\begin{tabular}{c|c|c}
\hline \multirow{4}{*}{ LM } & Correlação de Pearson & Marcha1a \\
\cline { 2 - 3 } & Sig.(p) &,$- 708\left(^{* *}\right)$ \\
\cline { 2 - 3 } & $\mathbf{N}$ & 0 \\
\hline
\end{tabular}

${ }^{* *}$ Correlação significante a 0,01 .

A correlação é satisfatória.

Tabela 39 - Comparação descritiva segundo os valores dos itens relacionados à marcha da HPN e MIF.

\begin{tabular}{|c|c|c|c|c|c|c|c|}
\hline & & \multicolumn{5}{|c|}{ Marcha1 } & \multirow{2}{*}{ Total } \\
\hline & & 0 & 1 & 2 & 3 & 4 & \\
\hline \multirow{13}{*}{ LM } & 2 & 0 & 1 & 2 & 5 & 22 & 30 \\
\hline & 3 & 0 & 0 & 2 & 1 & 0 & 3 \\
\hline & 4 & 0 & 2 & 3 & 3 & 0 & 8 \\
\hline & 5 & 0 & 2 & 1 & 0 & 0 & 3 \\
\hline & 6 & 0 & 5 & 7 & 1 & 2 & 15 \\
\hline & 7 & 0 & 2 & 1 & 2 & 0 & 5 \\
\hline & 8 & 0 & 7 & 0 & 0 & 0 & 7 \\
\hline & 9 & 1 & 6 & 0 & 0 & 0 & 7 \\
\hline & 10 & 1 & 8 & 4 & 4 & 0 & 17 \\
\hline & 11 & 0 & 4 & 5 & 1 & 0 & 10 \\
\hline & 12 & 1 & 10 & 1 & 0 & 0 & 12 \\
\hline & 13 & 1 & 2 & 0 & 0 & 0 & 3 \\
\hline & 14 & 1 & 0 & 0 & 0 & 0 & 1 \\
\hline \multicolumn{2}{|c|}{ Total } & 5 & 49 & 26 & 17 & 24 & 121 \\
\hline
\end{tabular}


Os valores mais altos na soma dos itens locomoção da MIF correspondem a valores mais baixos do item marcha da HPN e vice-versa (Tabela 39). 


\section{DISCUSSÃO}

Há diversas maneiras para mensurar construtos clínicos e validação de instrumentos psicométricos, que refletem a ausência de concordância no que se refere à teoria das medidas nas ciências entre os pesquisadores. Somado a isso, a falta de instrumentos de avaliação direcionados aos pacientes com HPN traduzidos e validados para o Português tem restringido as pesquisas nesse campo até hoje no Brasil.

Esta pesquisa teve como objetivo principal a validação para Língua Portuguesa da Escala de graduação do paciente com Hidrocefalia de Pressão Normal, construída a partir da tradução da "Grading Scale for Idiopathic Normal Pressure Hydrocephalus".

Seu construto foi elaborado visando quantificar o grau de comprometimento dos pacientes com HPN. A decisão pela validação dessa escala se deve ao fato de ser um instrumento que avalia, de forma simples e direcionada, o impacto do comprometimento na vida dessa população.

Após a sua tradução, a escala foi aplicada em uma amostra composta por cento e vinte e um pacientes, com maior frequência do gênero masculino, em concordância com os achados de $\mathrm{Mori}^{23}$, que pesquisou cento e vinte pacientes, e predomínio de igual gênero.

Com relação ao perfil dos pacientes, a média de idade média da população pesquisada foi de 71,1 anos $(\mathrm{dp}=2,8)$, variando de 35 e 92 anos, corroborando com os estudos de Ishikawa ${ }^{65}$, na qual a média de idade da população foi de 75 anos.

No tocante ao quadro clínico da HPN, de acordo com a literatura, trabalhos clássicos, como os de Hakim ${ }^{1}$, caracterizaram o quadro clínico por meio da tríade, distúrbio de marcha, incontinência urinária e demência. Vale salientar que a tríade não ocorre em todos os casos, como demonstrada nesta pesquisa.

Consoante com os achados, este estudo ratificou os dados da literatura com relação à prevalência de comprometimento ser na marcha, precedidos 
pela demência e incontinência urinária. Neste estudo, a maioria dos pacientes apresentava distúrbios motores (96,7\%), seguidos de distúrbios cognitivos (86,0\%) e esfincterianos $(79,3 \%)$.

Quanto à avaliação da confiabilidade e reprodutividade, ambas foram testadas por itens individualmente, pelo método teste-reteste, analisados pela medida de concordância Kappa.

Alguns fatores devem ser levados em consideração para minimizar a eventual instabilidade do construto clínico, o tempo teste-reteste (sete dias), uma vez que o tempo não poderia ser curto, a fim de que os pacientes pudessem sofrer influência pela recordação das respostas dadas inicialmente (respostas lembradas); tampouco ser longo, para que outras variáveis não pudessem interferir no padrão de respostas do sujeito em relação ao construto que estava sendo avaliado ${ }^{72,73}$.

$\mathrm{Na}$ avaliação intra-avaliador, a concordância foi de 0,80 para domínio marcha, 0,90 para demência e 0,87 para incontinência urinária, todos classificados como concordância excelente. A mesma classificação foi encontrada para a avaliação interavaliador, na qual se obteve pontuação de 0,91 para domínio marcha, 0,86 para demência e 0,87 para incontinência urinária, o que favorece o uso do instrumento em grande escala.

Apesar de não ter existir um padrão-ouro para avaliação da HPN, muitas escalas já desenvolvidas e utilizadas na literatura buscam avaliar a severidade da tríade da HPN. Entretanto, o principal problema enfrentado é a semelhança entre os itens dos domínios dessas, que, muitas vezes, dificulta a pontuação pelo entrevistador.

Kubo (2008) ${ }^{64}$ desenvolveu a Escala de graduação da evolução dos sintomas da HPN, posteriormente utilizada por Hashimoto $(2010)^{74}$, embora existam críticas com relação aos itens do domínio cognitivo que tendem a pontuar de forma dúbia amnésia, inatenção e desorientação. Ainda no domínio marcha, há uma inconsistência de classificação, pois a escala possui classificação diferente para o item desequilíbrio, mas sem distinção de distúrbio objetivo de marcha; marcha instável, entre outros.

Tous et al. $(2013)^{75}$ realizaram um estudo prospectivo por meio de escalas clínicas e achados radiológicos para avaliar 40 pacientes com HPN, 
tratados cirurgicamente. Os autores evidenciaram predominância no sexo masculino (62\%), média de idade 74,2 anos, comprometimento na tríade acentuado para marcha (92,5\%); seguido de $82,5 \%$ para cognitivo e $65,0 \%$ para esfincteriana, ratificando os dados deste estudo. As escalas selecionadas foram HPN crônica, Rankin e Pfeiffer. Os itens da escala de HPN envolviam, no domínio cognitivo, estado vegetativo; demência grave; problema de memória e mudança de caráter, problema de memória. Ainda no domínio marcha, pontuava-se o padrão normal; marcha normal, contudo estável, e instabilidade na marcha.

Outra escala existente, denominada HPN modificada, foi utilizada no estudo de Owler et al. (2003) ${ }^{76}$ para classificação clínica dos pacientes, entretanto, esta escala avalia de forma subjetiva as atividades de vida diária e o domínio esfincteriano apenas como presente ou ausente para distúrbio, assim, a escala validada por este estudo tende a ser mais completa e objetiva.

A respeito da avaliação da nova escala de HPN, esta teve suas pontuações comparadas com outras escalas validadas e consagradas na literatura, como a MIF, que tem sido utilizada desde valores totais de escores a fim de verificar independência funcional, como com valores estratificados denominados dimensões cognitiva e motora ${ }^{77}$. Na comparação com o escore total da MIF, verificou-se alta concordância dos achados, uma vez que os pacientes que apresentaram maior comprometimento na escala de HPN foram concomitantemente classificados com maiores comprometimentos na escala MIF, a comparação foi estatisticamente satisfatória $(p=-0,842)$.

$\mathrm{Na}$ comparação com escala de BERG, verificou-se que os pacientes que apresentaram maior comprometimento na escala de HPN também foram classificados com maiores comprometimentos na escala de equilíbrio de BERG, sendo também verdadeira e melhores pontuações em HPN, pontuaram de forma satisfatória na BERG, obtendo uma comparação estatisticamente satisfatória $(p=-0,803)$.

A comparação com escala de DGI verificou que os pacientes que apresentaram maior comprometimento na escala de HPN também foram classificados com maiores comprometimentos na escala DGI, sendo também 
verdadeira e melhores pontuações em HPN, pontuaram de forma satisfatória na DGI. A comparação foi considerada estatisticamente boa $(p=-0,694)$.

Quanto à comparação com a escala TUG, observou-se que os pacientes que apresentaram maior tempo na execução do teste, ou seja, pior resultado na pontuação TUG, foram os mesmos classificados com maiores comprometimentos na escala HPN, sendo também verdadeiro que os pacientes com melhores pontuações em HPN realizaram com menor tempo o teste (TUG) e maior mobilidade funcional $(p=-0,557)$.

Outro fator importante nesta validação foi a avaliação dos itens individuais da HPN, estes foram comparados com itens correspondentes da escala MIF, de maneira individualmente. Assim, obteve-se, de forma descritiva para o item incontinência urinária, concordância entre as escalas, pois os pacientes que apresentaram maior comprometimento na HPN foram classificados com maiores comprometimentos da MIF, com concordância estatisticamente satisfatória $(p=-0,884)$.

$\mathrm{O}$ item demência da HPN foi comparado à dimensão cognitiva da MIF, evidenciando uma concordância estatisticamente boa ( $p=-0,741)$, porquanto os pacientes com maior comprometimento de HPN receberam maior pontuação e, por consequência, maior comprometimento na dimensão cognitiva da MIF.

Em relação ao item marcha da HPN, foi realizada a comparação com a locomoção da MIF, os pacientes que tiveram maior comprometimento na escala de HPN foram classificados com maiores comprometimentos na locomoção da MIF, sendo também verdadeiro que melhores pontuações em HPN para este item pontuaram de forma satisfatória na MIF $(p=-0,708)$.

Considera-se, assim, que este estudo é um contributo relevante para a área da saúde, quer no contexto clínico, quer científico, ao disponibilizar um recurso para a avaliação específica do paciente com Hidrocefalia de Pressão Normal acerca da alteração do seu estado de saúde, que pode ajudar a avaliar os benefícios na comparação dos resultados entre intervenções e/ou na identificação de diferenças clinicamente importantes na evolução do quadro apresentado. 


\section{CONCLUSÃO}

A versão "Grading Scale for Idiopathic Normal Pressure Hydrocephalus" foi traduzida e validada com sucesso para a Língua Portuguesa, como "Escala de Graduação do Paciente com Hidrocefalia de Pressão Normal", de acordo com o resultado da análise final de suas propriedades de medida.

O presente estudo apresentou correlação estatisticamente significante da escala HPN traduzida com as escalas: Medida de independência funcional (MIF), índice de Marcha dinâmica (DGl), teste de equilíbrio de Berg (BERG) e "Timed Up and Go" (TUG); E satisfatória concordância interavaliador e intraavaliador na análise da pontuação na versão traduzida.

Sendo assim, pela sua simplicidade e brevidade, torna-se um instrumento prático e disponível para utilização em pesquisas clínicas e em ensaios epidemiológicos no Brasil. 


\section{AnEXos}




\section{ANEXOS}

ANEXO A: Medida de independência funcional.

\begin{tabular}{|c|c|c|}
\hline \multirow{12}{*}{ MIF MOTORA } & \multirow{6}{*}{ AUTOS CUIDADOS } & Alimentação \\
\hline & & Higiene Pessoal \\
\hline & & Banho: limpeza do corpo \\
\hline & & $\begin{array}{l}\text { Vestir metade superior do } \\
\text { corpo }\end{array}$ \\
\hline & & $\begin{array}{c}\text { Vestir metade inferior do } \\
\text { corpo }\end{array}$ \\
\hline & & Uso do vaso sanitário \\
\hline & \multirow{2}{*}{$\begin{array}{c}\text { CONTROLE } \\
\text { ESFINCTERIANO }\end{array}$} & Controle da urina \\
\hline & & Controle das fezes \\
\hline & \multirow{2}{*}{ MOBILIDADE } & Vaso sanitário \\
\hline & & Banheira ou chuveiro \\
\hline & \multirow{2}{*}{ LOCOMOÇÃO } & Andar \\
\hline & & Escadas \\
\hline \multirow{5}{*}{ MIF COGNITIVA } & \multirow{2}{*}{ COMUNICAÇÃO } & Compreensão \\
\hline & & Expressão \\
\hline & \multirow{3}{*}{ COGNIÇÃO SOCIAL } & Interação Social \\
\hline & & Resolução de problemas \\
\hline & & Memória \\
\hline
\end{tabular}

( ) Escore Total $($ Máximo $=126)$

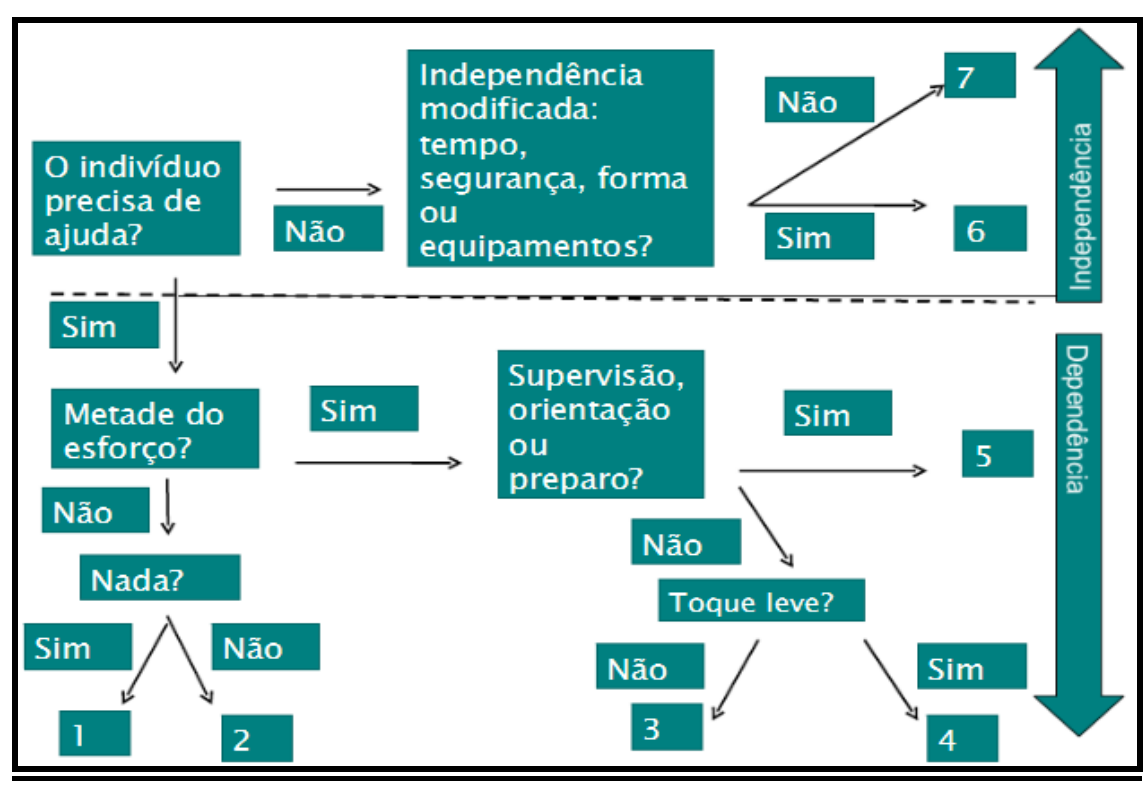




\section{Pontuacão:}

7- Independência Completa: todas as tarefas são realizadas de forma segura, dentro de um tempo razoável.

6-Independência modificada: capaz de realizar tarefas com recursos auxiliares, necessitando de tempo, porém realiza de forma segura e totalmente independente.

5- Supervisão: Necessita de supervisão ou comandos verbais ou modelos para realizar a tarefa sem necessidade de ajuda somente o preparo da tarefa quando necessário

4-Assistência mínima: mínima quantidade de assistência, um simples tocar, possibilitando a execução da atividade (realiza $75 \%$ do esforço necessário na tarefa).

3- Assistência moderada: moderada quantidade de assistência, mais do que simplesmente tocar (realiza 50\% do esforço necessário na tarefa).

2- Alta assistência: utiliza menos que $50 \%$ do esforço necessário para completar a tarefa, mas não necessita auxílio total.

1- Assistência total: é necessária ou a tarefa não é realizada. Utiliza-se menos que $25 \%$ do esforço necessário para realizar tarefa. 
ANEXO B: Escala de Equilíbrio de Berg

\section{Posição sentada para posição em pé}

( 4 ) capaz de levantar-se sem utilizar as mãos e estabilizar-se independentemente

( 3 ) capaz de levantar-se independentemente utilizando as mios

( 2 ) capaz de levantar-se utilizando as mãos após diversas tentativas

( 1 ) necessita de ajuda mínima para levantar-se ou estabilizar-se

( 0 ) necessita de ajuda moderada ou máxima para levantar-se

\section{Permanecer em pé sem apoio}

( 4 ) capaz de permanecer em pé com segurança por 2 minutos

( 3 ) capaz de permanecer em pé por 2 minutos com supervisão

(2) capaz de permanecer em pé por 30 segundos sem apoio

( 1 ) necessita de várias tentativas para permanecer em pé por 30 segundos sem apoio

( 0 ) incapaz de permanecer em pé por 30 segundos sem apoio. Se o paciente for capaz de permanecer em pé por 2 minutos sem apoio, dê o número total de pontos para o item número 3. Continue com o item número 4.

\section{Permanecer sentado sem apoio nas costas, mas com os pés apoiados no chão ou num banquinho}

( 4 ) capaz de permanecer sentado com segurança e com firmeza por 1 minutos

( 3 ) capaz de permanecer sentado por 2 minutos sob supervisão

( 2 ) capaz de permanecer sentado por 30 segundos

( 1 ) capaz de permanecer sentado por 10 segundos

( 0 ) incapaz de permanecer sentado sem apoio durante 10 segundos

\section{Posição em pé para posição sentada}

( 4 ) senta-se com segurança com uso mínimo das mãos

( 3 ) controla a descida utilizando as mios

( 2 ) utiliza a pane posterior das pernas contra a cadeira para controlar a descida

( 1 ) senta-se independentemente, mas tem descida sem controle

( 0 ) necessita de ajuda para sentar-se

\section{Transferências}

( 4 ) capaz de transferir-se com segurança com uso mínimo das mãos

( 3 ) capaz de transferir-se com segurança com o uso das mãos

( 2 ) capaz de transferir-se seguindo orientações verbais c/ou supervisão

( 1 ) necessita de uma pessoa para ajudar

( 0 ) necessita de duas pessoas para ajudar ou supervisionar para realizar a tarefa com segurança

\section{Permanecer em pé sem apoio com os olhos fechados}

( 4 ) capaz de permanecer em pé por 10 segundos com segurança

( 3 ) capaz de permanecer em pé por 10 segundos com supervisão

( 2 ) capaz de permanecer em pé por 3 segundos

( 1 ) incapaz de permanecer com os olhos fechados durante 3 segundos, mas mantém-se em pé

( 0 ) necessita de ajuda para não cair 
7. Permanecer em pé sem apoio com os pés juntos

( 4 ) capaz de posicionar os pés juntos independentemente e permanecer por 1 minuto com segurança

( 3 ) capaz de posicionar os pés juntos independentemente e permanecer por 1 minuto com supervisão

( 2 ) capaz de posicionar os pés juntos independentemente e permanecer por 30 segundos

( 1 ) necessita de ajuda para posicionar-se, mas é capaz de permanecer com os pés juntos durante 15 segundos

( 0 ) necessita de ajuda para posicionar-se e é incapaz de permanecer nessa posição por 15 segundos

\section{Alcançar a frente com o braço entendido permanecendo em pé}

( 4 ) pode avançar à frente mais que $25 \mathrm{~cm}$ com segurança

( 3 ) pode avançar à frente mais que $12,5 \mathrm{~cm}$ com segurança

( 2 ) pode avançar à frente mais que $5 \mathrm{~cm}$ com segurança

( 1 ) pode avançar à frente, mas necessita de supervisão

( 0 ) perde o equilíbrio na tentativa, ou necessita de apoio externo

\section{Pegar um objeto do chão a partir de uma posição em pé}

( 4 ) capaz de pegar o chinelo com facilidade e segurança

( 3 ) capaz de pegar o chinelo, mas necessita de supervisão

( 2 ) incapaz de pegá-lo, mas se estica até ficar a $2-5 \mathrm{~cm}$ do chinelo e mantém o equilíbrio independentemente

( 1 ) incapaz de pegá-lo, necessitando de supervisão enquanto está tentando

( 0 ) incapaz de tentar, ou necessita de ajuda para não perder o equilíbrio ou cair

10. Virar-se e olhar para trás por cima dos ombros direito e esquerdo enquanto permanece em pé

( 4 ) olha para trás de ambos os lados com uma boa distribuição do peso

( 3 ) olha para trás somente de um lado o lado contrário demonstra menor distribuição do peso

( 2 ) vira somente para os lados, mas mantém o equilíbrio

( 1 ) necessita de supervisão para virar

( 0 ) necessita, de ajuda para não perder o equilíbrio ou cair

\section{Girar 360 graus}

( 4 ) capaz de girar 360 graus com segurança em 4 segundos ou mãos

( 3 ) capaz de girar 360 graus com segurança somente para um lado em 4 segundos ou menos

( 2 ) capaz de girar 360 graus com segurança, mas lentamente

( 1 ) necessita de supervisão próxima ou orientações verbais

( 0 ) necessita de ajuda enquanto gira 
12. Posicionar os pés alternadamente ao degrau ou banquinho enquanto permanece em pé sem apoio

( 4 ) capaz de permanecer em pé independentemente e com segurança, completando 8 movimentos em 20 segundos

( 3 ) capaz de permanecer em pé independentemente e completar 8 movimentos em mais que 20 segundos

( 2 ) capaz de completar 4 movimentos sem ajuda

( 1 ) capaz de completar mais que 2 movimentos com o mínimo de ajuda

( 0 ) incapaz de tentar, ou necessita de ajuda para não cair

\section{Permanecer em pé sem apoio com um pé à frente}

( 4 ) capaz de colocar um pé imediatamente à frente do outro, independentemente, e permanecer por 30 segundos

( 3 ) capaz de colocar um pé um pouco mais à frente do outro e levemente para o lado.Independentemente e permanecer por 30 segundos

( 2 ) capaz de dar um pequeno passo, independentemente. e permanecer por 30 segundos

( 1 ) necessita de ajuda para dar o passo, porém permanece por 15 segundos

( 0 ) perde o equilíbrio ao tentar dar um passo ou ficar de pé

\section{Permanecer em pé sobre uma perna}

( 4 ) capaz de levantar uma perna independentemente e permanecer por mais que 10segundos

( 3 ) capaz de levantar uma perna independentemente e permanecer por 5-10 segundos

( 2 ) capaz de levantar uma perna independentemente e permanecer por 3 ou 4 segundos

( 1 ) tenta levantar uma perna, mas é incapaz de permanecer por 3 segundos, embora permaneça em pé independentemente

( 0 ) incapaz de tentar, ou necessita de ajuda para não cair

( ) Escore Total (Máximo = 56) 


\section{ANEXO C: Índice de Marcha Dinâmica}

1. Marcha em superfície plana

Instruções: Ande em sua velocidade normal, daqui ate a próxima marca (6 metros). Pontuação: Marque a menor categoria que se aplica:

(3) Normal: Anda 6 metros, sem dispositivos de auxilio, em boa velocidade, sem evidencia de desequilíbrio, marcha em padrão normal.

(2) Comprometimento leve: Anda 6 metros, velocidade lenta, marcha com mínimos desvios, ou utiliza dispositivos de auxilio a marcha.

(1) Comprometimento moderado: Anda 6 metros, velocidade lenta, marcha em padrão anormal, evidencia de desequilíbrio.

(0) Comprometimento grave: Não consegue andar 6 metros sem auxilio, grandes desvios da marcha ou desequilíbrio.

1. Mudanças na velocidade da marcha

Instruções: comece a andar na sua velocidade normal (durante $1.5 \mathrm{~m}$ ), e quando eu disser 'agora', ande o mais rápido possível que puder por mais $1.5 \mathrm{~m}$. Quando eu disser 'devagar', ande o mais lentamente que conseguir $(1.5 \mathrm{~m})$. Pontuação: marque a categoria inferior que se aplica.

(3) Normal: Capaz de mudar a velocidade da marcha de forma uniforme, sem perda de equilíbrio ou desvio da marcha. Mostra uma diferença significativa nas velocidades entre o normal, o rápido e o lento.

(2) Comprometimento mínimo: Consegue mudar a velocidade mas demonstra desvios mínimos da marcha, ou não ha desvios, mas ele e incapaz de obter uma mudança significativa na velocidade ou utiliza um acessório.

(1) Comprometimento moderado: Realiza somente pequenos ajustes na velocidade da marcha, ou apresenta uma alteração com importantes desvios, ou alterada a velocidade associada a desvios significativos da marcha, ou altera a velocidade com perda do equilíbrio, mas e capaz de recupera-la e continuar andando.

(0) Comprometimento severo: Não consegue mudar a velocidade ou perde o equilíbrio e procura apoio na parede ou tem que ser pego.

2. Marcha com rotação horizontal da cabeça

Instruções: Comece a andar no ritmo normal. Quando eu disser 'olhe para a direita', continue andando reto, vire a cabeça para a direita. Continue olhando para o lado direito ate que eu diga 'olhe para a esquerda', então continue andando reto e vire a cabeça para a esquerda.

Mantenha a cabeça nesta posição ate que eu diga "olhe para a frente", então continue andando

reto mas volte a sua cabeça para a posição central.

Pontuação: marque a categoria inferior que se aplica

(3) Normal: Executa rotações uniformes da cabeça, sem nenhuma mudança na marcha.

(2) Comprometimento mínimo: Executa rotações uniformes da cabeça, com uma ligeira mudança na velocidade da marcha (isto é, interrupção mínima no trajeto uniforme da marcha ou usa um acessório para andar). 
(1) Comprometimento moderado: Executa rotações uniformes da cabeça, com uma moderada mudança na velocidade da marcha, começa a andar mais lentamente, vacila mas se recupera, consegue continuar andando.

(0) Comprometimento severo: Executa as tarefas com interrupções severas da marcha (isto e, desvia $15^{\circ}$ fora do trajeto, perde o equilíbrio, para, tenta segurar-se na parede)

4. Marcha com movimentos verticais da cabeça

Instruções: Comece a andar no ritmo normal. Quando eu disser 'olhe para cima', continue andando reto, mas incline a cabeça para cima. Continue olhando para cima ate que eu diga 'olhe para baixo', então continue andando reto e vire a cabeça para baixo. Mantenha a cabeça nesta posição ate que eu diga 'olhe para a frente', então continue andando reto mas volte a sua cabeça para a posição central. Pontuação: marque a categoria inferior que se aplica.

(3) Normal: Executa rotações uniformes da cabeça, sem nenhuma mudança na marcha.

(2) Comprometimento mínimo: Executa as tarefas com uma ligeira mudança na velocidade da marcha (isto e, interrupção mínima no trajeto uniforme da marcha ou usa um acessório para andar).

(1) Comprometimento moderado: Executa as tarefas com uma moderada mudança na velocidade da marcha, começa a andar mais lentamente, vacila mas se recupera, consegue continuar andando.

(0) Comprometimento severo: Executa as tarefas com interrupções severas da marcha (isto e, vacila $15 \mathrm{o}$ fora do trajeto, perde o equilíbrio, para, tenta segurar-se na parede).

5. Marcha e rotação

Instruções: Comece a andar no ritmo normal. Quando eu disser "vire-se e pare", vire o mais rápido que puder para a direção oposta e pare.

Pontuação: marque a categoria inferior que se aplica.

(3) Normal: Consegue virar com segurança dentro de 3 segundos e para rapidamente, sem nenhuma perda do equilíbrio.

(2) Comprometimento mínimo: Consegue virar com segurança $<3$ segundos e para sem nenhuma perda do equilíbrio.

(1) Comprometimento moderado: Vira lentamente, precisa de dicas verbais, precisa dar vários passos curtos para recuperar o equilíbrio após virar ou parar.

(0) Comprometimento severo: Não consegue girar com segurança, precisa de ajuda para virar e parar.

6. Passar por cima de um obstáculo

Instruções: Comece a nadar em sua velocidade normal. Quando chegar a caixa de sapatos, passe por cima dela (não ao redor dela) e continue andando.

Pontuação: marque a categoria inferior que se aplica.

(3) Normal: Capaz de passar por cima da caixa sem mudar a velocidade da marcha; não há evidencia de desequilíbrio.

(2) Comprometimento mínimo: Capaz de passar por cima da caixa, mas precisa reduzir a velocidade e ajustar os passos para ter mais segurança.

(1) Comprometimento moderado: E capaz de passar por cima da caixa, mas precisa parar e depois recomeçar. Pode precisar de dicas verbais. 
(0) Comprometimento severo: Não consegue executar sem ajuda.

7. Andar ao redor de obstáculos

Instruções: Comece a andar na sua velocidade normal. Quando chegar ao primeiro cone (1.80 $\mathrm{m}$ de distancia), contorne-o pelo lado direito. Quando chegar ao segundo $(1.80 \mathrm{~m}$ após o primeiro), contorne-o pela esquerda.

Pontuação: marque a categoria inferior que se aplica

(3) Normal: E capaz de andar ao redor dos cones com segurança, sem mudar a velocidade da marcha; não ha evidencia de desequilíbrio.

(2) Comprometimento mínimo: E capaz de andar ao redor de ambos os cones, mas precisa reduzir a velocidade da marcha e ajustar os passos para passar por eles.

(1) Comprometimento moderado: E capaz de passar pelos cones, mas precisa reduzir significativamente a velocidade da marcha para realizar a tarefa.

(0) Comprometimento severo: Incapaz de passar pelos cones, tropeça neles e precisa de ajuda física.

8. Degraus

Instruções: Suba estes degraus da maneira que você faz em casa (isto e, usando o corrimãos se necessário). Quando chegar ao topo, vire e desça novamente.

Pontuação: marque a categoria inferior que se aplica

( 3 ) Normal: Alternando os pés, sem usar o corrimão

( 2 ) Comprometimento mínimo: Alternando os pés, mas precisa usar o corrimão.

( 1 ) Comprometimento moderado: Coloca os 2 pés no degrau, precisa usar o corrimão.

( 0 ) Comprometimento severo: Não consegue fazer de forma segura.

( ) Escore Total $($ Máximo $=24)$ 
ANEXO D: "Grading scale for NPH"

\section{Grading Scale for NPH}

\section{Gait disturbance}

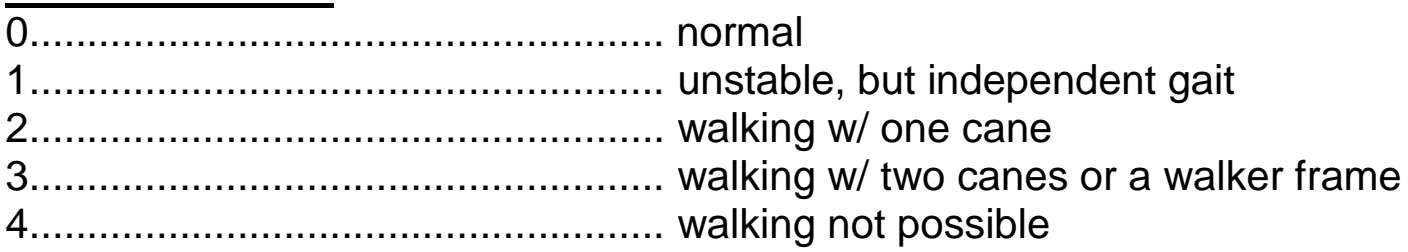

\section{Dementia}

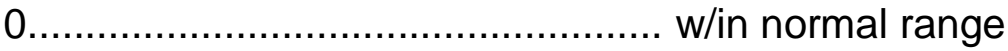

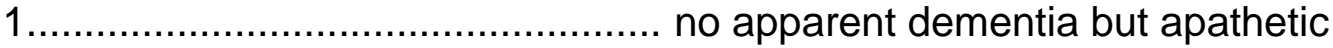

.

home

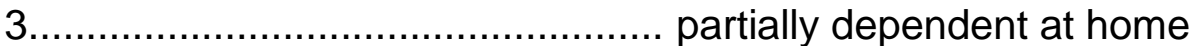

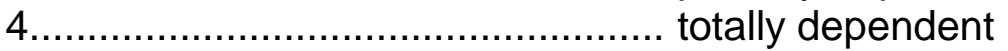

\section{Urinary incontinence}

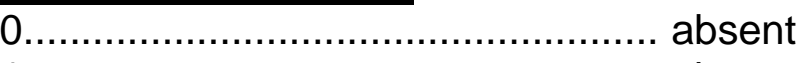

1

urgency

2.............................................. sometimes only at night

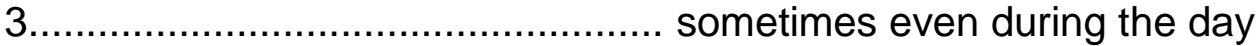

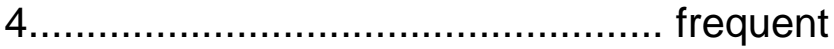


ANEXO E: Escala de Graduação de HPN.

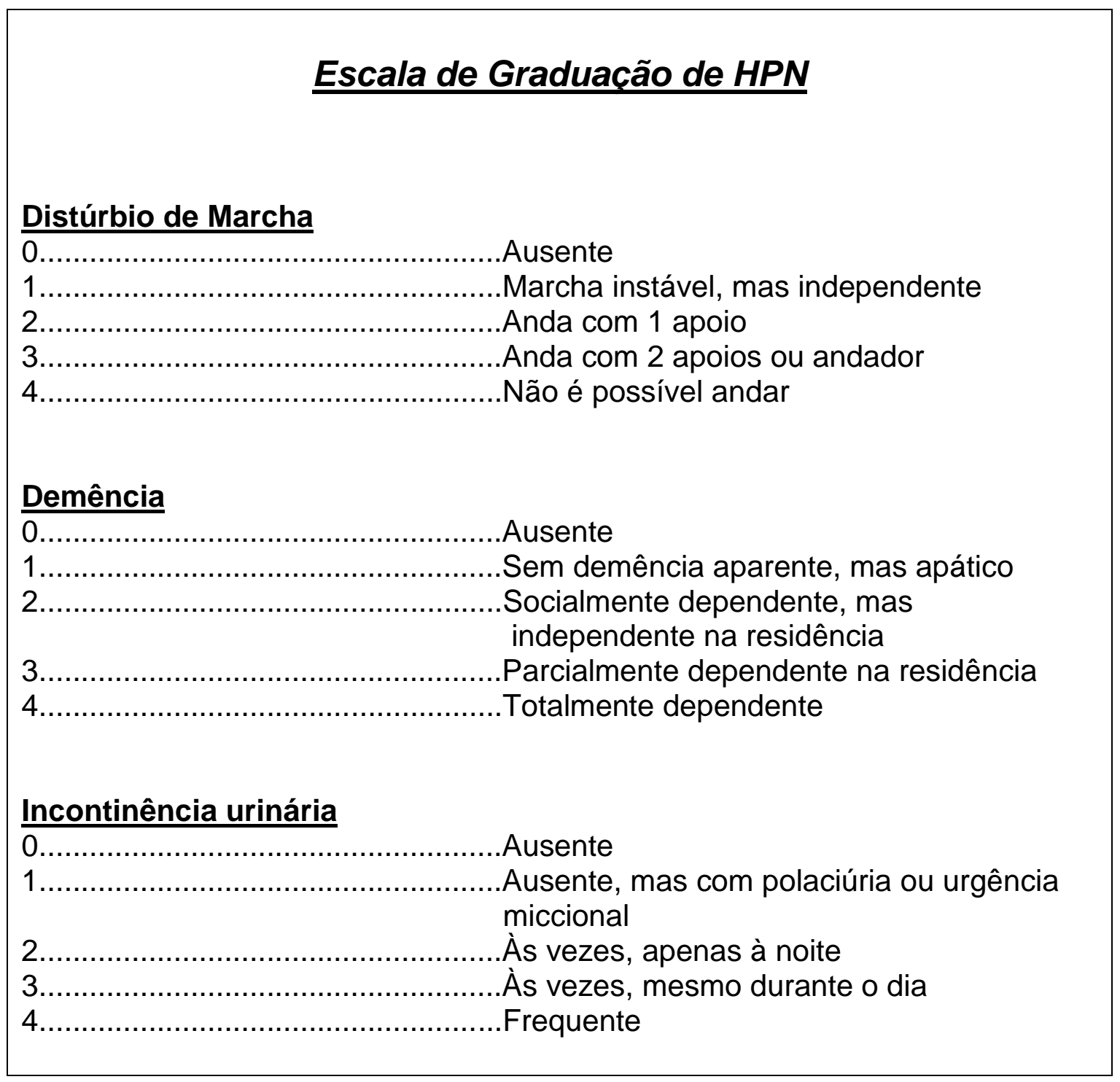


ANEXO F: Aprovação do Projeto de Pesquisa pela CAPpesq do HCFMUSP.

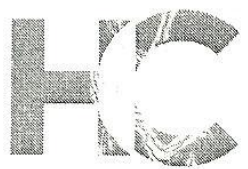

\section{APROVAÇÃOO}

A Comissão de Éfica para Anális de Projetos de: Fesquisa CAPPesa da Diretaria Clínica do Hospital das Clíniccis e da Faculdade de Medicina de Universidade de São Paulo, em sesscio de: 07/(1)7/2010. AProvou O Protocolo de Pesquisa no 0369/10, intitulado: "VALLDAÇĀOA NA LÍNGUA PORTUGUES: DA ESCALA DE GRADUAÇÃO DO P/:CIENTI: COM HIDKOCEFALIA DE PRESSÃO NORMAL." apresentado pelo Departainento cie inÉs ROLONIA, riclusive O Termo de Co:iseritimeintc Livre e Esclarecido.

Cabe ao pesquisador elciborar e aruresentar à CAPPesq, os relatórios parciais e fincii sobre a pesquisa (Resolução do. Conselho Nacional de Saúde n 196: de 10/10/1996, inciso !X.2, letra "c"!.

Pesquisador (a) Responsćvel: Prof.Dr. Mcinoel Jacoisen Teizeira / Lir. Fernando Campos Gomes Pint:

Pesquisador (a) Executante: Maria lzabel Romāo Lones

CAFresci, 0\% da Julho de 2010

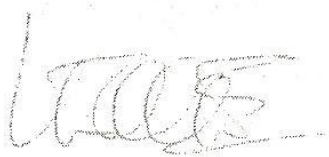

PROF. DR. CLAUDIO LIIONE

Vice-Presiderite da Comissāo Étiza para Análise de Proiotos de Pesquiso 
ANEXO G: Termo de Consentimento Livre e Esclarecido

HOSPITAL DAS CLÍNICAS DA FACULDADE DE MEDICINA DA UNIVERSIDADE DE SÃO PAULO-HCFMUSP

\section{DADOS DE IDENTIFICAÇÃO DO SUJEITO DA PESQUISA OU RESPONSÁVEL LEGAL}

1. NOME:

DOCUMENTO DE IDENTIDADE $\mathrm{N}^{\circ}$ : SEXO:.$M \square F \square$ DATA NASCIMENTO: ........................ ENDEREÇO

BAIRRO: CEP:. TELEFONE: DDD ( CIDADE $\mathrm{N}^{\mathrm{o}}$ APTO:

2.RESPONSÁVEL LEGAL NATUREZA (grau de parentesco, tutor, curador etc.)

DOCUMENTO DE IDENTIDADE : SEXO: $\mathrm{M} \square \mathrm{F} \square$ DATA NASCIMENTO.: ...................... ENDEREÇO: BAIRRO: CEP:. TELEFONE:DDD ( ... $\mathrm{N}^{\mathrm{o}}$ APTO: CIDADE:

\section{DADOS SOBRE A PESQUISA}

1. TÍTULO DO PROTOCOLO DE PESQUISA: Validação para Língua Portuguesa da escala de graduação do Paciente com Hidrocefalia de Pressão Normal.

2. PESQUISADOR : Dr Fernando Campos Gomes Pinto

CARGO/FUNÇÃO: médico INSCRIÇÃO CONSELHO REGIONAL No ._CRM 90797 UNIDADE DO HCFMUSP: Ipq

3. AVALIAÇÃO DO RISCO DA PESQUISA:

$\begin{array}{lll}\text { RISCO MÍNIMO } & \square & \text { RISCO MÉDIO } \\ \text { RISCO BAIXO } & \mathrm{X} & \text { RISCO MAIOR }\end{array}$

4.DURAÇÃO DA PESQUISA: 36 meses 


\section{HOSPITAL DAS CLÍNICAS DA FACULDADE DE MEDICINA DA UNIVERSIDADE DE SÃO PAULO-HCFMUSP}

01 - Essas informações estão sendo fornecidas para sua participação voluntária neste estudo, que visa validar para a Língua Portuguesa um questionário com perguntas para avaliar os pacientes com hidrocefalia.

02 - Você será avaliado por duas Fisioterapeutas em um único dia, no Ambulatório de Hidrodinâmica do Hospital das Clínicas. Elas farão perguntas sobre o caminhar, controle da urina, sobre seu comportamento e suas atividades em casa, como vestir roupa e alimentar-se. Depois apenas uma fisioterapeuta fará teste como pedir para você pegar um caderno no chão, levantar da cadeira, equilibrar-se em apenas um pé para observar o seu equilíbrio corporal e após, verá a forma como você anda.

03 - Há risco baixo na avaliação realizada pela Fisioterapeuta, por pedir para você andar. Será desenvolvida em no máximo por quinze minutos cada avaliação, com total de quarenta e cinco minutos, com a presença dos seus familiares.

04 - Em qualquer etapa do estudo, você terá acesso aos profissionais responsáveis pela pesquisa para esclarecimento de eventuais dúvidas. Os principais investigadores são Dr Manoel Jacobsen e Dr Fernando Campos Gomes Pinto, que pode ser encontrado no endereço Av. Dr. Enéas de Carvalho Aguiar, 255 Cerqueira César, CEP 05403-000, São Paulo - Brasil. Telefone (0xx11) 8203-5550. Se você tiver alguma consideração ou dúvida sobre a ética da pesquisa, entre em contato com o Comitê de Ética em Pesquisa (CEP) - Rua Ovídio Pires de Campos, 225 - $5^{\circ}$ andar - tel: 3069-6442 ramais 16, 17, 18 ou 20, FAX: 3069-6442 ramal 26 - E-mail: cappesq@hcnet.usp.br

05 - É garantida a liberdade da retirada de consentimento a qualquer momento e deixar de participar do estudo, sem qualquer prejuízo à continuidade de seu tratamento na Instituição;

06 - As informações obtidas serão analisadas em conjunto com outros pacientes, não sendo divulgada a identificação facial ou dados pessoais de nenhum paciente;

07 - Direito de ser mantido atualizado sobre os resultados parciais das pesquisas, quando em estudos abertos, ou de resultados que sejam do conhecimento dos pesquisadores;

08 - Não há despesas pessoais para o participante em qualquer fase do estudo, incluindo exames e consultas. Também não há compensação financeira relacionada à sua participação.

09 - Compromisso do pesquisador de utilizar os dados e o material coletado somente para esta pesquisa. 
Acredito ter sido suficientemente informado a respeito das informações que li ou que foram lidas para mim, descrevendo o estudo "Validação para Língua Portuguesa da escala de graduação do Paciente com Hidrocefalia de Pressão Normal”.

Eu discuti com o Dr. Fernando Campos Gomes Pinto sobre a minha decisão em participar nesse estudo. Ficaram claros para mim quais são os propósitos do estudo, os procedimentos a serem realizados, seus desconfortos e riscos, as garantias de confidencialidade e de esclarecimentos permanentes. Ficou claro também que minha participação é isenta de despesas e que tenho garantia do acesso a tratamento hospitalar quando necessário. Concordo voluntariamente em participar deste estudo e poderei retirar o meu consentimento a qualquer momento, antes ou durante o mesmo, sem penalidades ou prejuízo ou perda de qualquer benefício que eu possa ter adquirido, ou no meu atendimento neste Serviço.

Assinatura do paciente/representante legal

Data

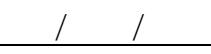

Assinatura da testemunha

Data $\frac{1}{1}$

para casos de pacientes analfabetos ou semianalfabetos.

(Somente para o responsável do projeto)

Declaro que obtive de forma apropriada e voluntária o Consentimento Livre e Esclarecido deste paciente ou representante legal para a participação neste estudo. 
8 REFERÊNCIAS 


\section{REFERÊNCIAS}

1. Hakim S, Adams RD. The special clinical problem of symptomatic hydrocephalus with normal cerebrospinal fluid pressure. Observations on cerebrospinal fluid hydrodynamics. J Neurol Sci. 1965;2(4):307-27.

2. Hakim S. Some observations on CSF pressure: hydrocephalic syndrome in adults with "normal" CSF pressure [Tese]. Bogotá: Universidade Javeriana da Faculdade de Medicina; 1964.

3. Bateman GA, Loiselle AM, Can MR. Measurement of intracranial hydrodynamics and compliance differentiate which patient with idiopathic normal pressure hydrocephalus will improve following shunt insertion?. Acta Neurochir (Wien). 2007;149(5):455-62.

4. Chang CC, Asada H, Mimura T, Suzuki S. A prospective study of cerebral blood flow and cerebrovascular reactivity to acetazolamide in 162 patients with idiopathic normal-pressure hydrocephalus. I Neurosurg. 2009;111(3):610-7.

5. Marmarou A, Young HF, Aygok GA, Sawauchi S, Tsuji O, Yamamoto T, Dunbar J. Diagnosis and management of idiopathic normal-pressure hydrocephalus: a prospective study in 151 patients. J Neurosurg. 2005;102(6):987-97.

6. Wallenstein MB, Mckhann GM, Salomon H. Discovery of normal-pressure hydrocephalus. Neurosurgery. 2010;67(1):155-9.

7. Hebb AO, Cusimano MD. Idiopathic normal pressure hydrocephalus: A systematic review of diagnosis and outcome. I Neurosurg. 2001;49(5):1166-84.

8. Adams RD, Fischer CM, Hakim S, Ojemann RG, Sweet WH. Symptomatic occult hydrocephalus with "normal" cerebrospinal-fluid pressure. $N$ Engl $J$ Med. 1965;273:117-26.

9. Graff-Radford NR. Normal pressure hydrocephalus. Neurol Clin. 2007;25(3):809-32.

10. Marmarou A, Bergsneider M, Klinge P, Relkin N, Black PM. The value of supplemental prognostic tests for the preoperative assessment of idiopathic normal-pressure hydrocephalus. Neurosurgery. 2005;57(3Suppl):S17-28. 
11. Krauss JK, von Stuckrad-Barre SF. Clinical aspects and biology of normal pressure hydrocephalus. Handb Clin Neurol. 2008;89:887-902.

12. Poca MA, Sahuquillo J, Mataro M. Update on diagnosis and treatment of normotensive hydrocephalus (chronic hydrocephalus of the adult). Neurologia. 2001;16(8):353-69.

13. Vacca V. Diagnosis and treatment of idiopathic normal pressure hydrocephalus. J Neurosci Nurs. 2007;39(2):107-11.

14. Ishii M, Akiguchi I. Recovery process of gait disturbance after ventriculoperitoneal shunt in patients with idiopathic normal pressure hydrocephalus. J Phys Ther Sci. 2007;19(3):183-8.

15. Melato L, Bigal ME, Speciali JG. Hidrocefalia de pressão normal: avaliação de cinco anos de experiência e revisão de literatura. Medicina (Rib. Preto). 2000;33:499-505.

16. Fernandez $P$, Munoz MA, Ortega RE. Hidrocefalia cronica del adulto: analisis descritivo de 36 casos. Cuad Cir. 2006;20:43-7.

17. Malm J, Eklund A. Idiopathic normal pressure hydrocephalus. Pract Neurol. 2006; 6:14-27.

18. Hellstrom P, Edsbagge M, Blomsterwall E, Archer T, Tisell M, Tullberg M, Wikkelso C. Neuropsychologic effects of shunt treatment in idiopathic normal pressure hydrocephalus. Neurosurgery. 2008;63(3):527-36.

19. Raftopoulos C, Deleval J, Chaskis C, Leonard A, Cantraine F, Desmyttere F, Clarysse S, Brotchi J. Cognitive recovery in Idiopathic normal pressure hydrocephalus: A prospective study. Neurosurgery. 1994;35(3):397-404.

20. Gangemi M, Maiuri F, Naddeo M, Godano U, Mascari C, Broggi G, Ferroli P. Endoscopic third ventriculostomy in idiopathic normal pressure hydrocephalus: an Italian multicenter study. Neurosurgery. 2008;63(1):627.

21 Miranda FL, Tornai JB, Lopes MIR. Caracterização da marcha do paciente com hidrocefalia de pressão normal. In: Pinto FCG. Hidrocefalia de pressão normal. São Paulo:Segmento Farma Editores, 1ed, 2012. p. 85103.

22. Blomsterwall E, Svantesson U, Carlsson U, Tullberg M, Wikkelsö C. Postural disturbance in patients with normal pressure hydrocephalus. Acta Neurol Scand. 2000;102(5):284-91.

23. Mori K. Management of idiopathic normal-pressure hydrocephalus: a multiinstitutional study conducted in Japan. J Neurosurg. 2001;95(6):9703. 
24. Ness D. Physical therapy management for conversion disorder: case series. J Neurol Phys Ther. 2007;31(1):30-9.

25. Feick D, Sickmond J, Liu L, Metellus P, Williams M, Rigamonti D, HillBriggs F. Sensitivity and predictive value of occupational and physical therapy assessments in the functional evaluation of patients with suspected normal pressure hydrocephalus. $J$ Rehabil Med. 2008;40(9):715-20.

26. Fraser JJ, Fraser C. Gait Disorder is the cardinal sign of normal pressure hydrocephalus: a case study. J Neurosci Nurs. 2007;39(3):132-4

27. Alisky J. Normal pressure hydrocephalus co-existing with a second dementia disorder. Neuropsychiatr Dis Treat. 2008;4(1):301-4.

28. Verres M, Selman WR. Management of normal pressure hydrocephalus. Am Fam Physician. 2004;70:1071-8.

29. Bradley WG. Normal pressure hydrocephalus: New concepts on etiology and diagnosis. AJNR Am J Neuroradiol. 2000;21(9):1586-90.

30. Tsunoda A, Mitsuoka $H$, Bandai $H$, Endo $H$, Arai $H$, Sato K. Intracranial crerebrospinal fluid measurement studies in suspected idiopathic normal pressure hydrocephalus, secundary normal pressure hydrocephalus, and brain atrophy. J Neurol Neurosurg Psychiatry. 2002,73(5):552-5.

31. Bech-Azeddine R, Hogh P, Juhler M, Gjerris F, Waldemar G. Idiopathic normal pressure hydrocephalus: clinical comorbidity correlated with cerebral biopsy findingsand outcome of cerebrospinal fluid shunting. $J$ Neurol Neurosurg Psychiatry. 2007;78(2):157-61.

32. Kiefer $M$, Unterberg $A$. The differential diagnosisand treatment of normal pressure hydrocephalus. Dtsch Arztebl Int. 2012;109(1-2):15-26.

33. Hattori E. White mattes alteration in idiopathic normal pressure hydrocephalus: tract-based spatial statistics study. Am J Neuroradiol. 2012;33:97-103.

34. Mesure S, Donnet A, Azulay JP, Pouget J, Grisoli F. Postural and locomotor evaluation of normal pressure hydrocephalus: a case report. Rev Neurol (Paris). 2001;157(11 Pt1):1416-9.

35. Blomsterwall E, Bilting M, Stephensen H, Wikkelsö C. Gait abnormality is not the only motor disturbance in normal pressure hydrocephalus. Scand $J$ Rehabil Med. 1995;27(4):205-9.

36. Estanol BV. Gait apraxia in communicating hydrocephalus. J Neurol Neurosurg Psychiatry. 1981;44(4):305-8.

37. Czerwosz L, Szczepek E, Sokolowska B, Jozwik A, Dudzinski K, Jurkiewicz J, Czernicki Z. Recognition of gait disturbances in patients with 
normal pressure hydrocephalus using a computer dynography system. $J$ Physiol Pharmacol. 2008;59(Suppl 6):201-7.

38. Ishikawa $\mathrm{M}$; Guideline Committe for Idiopathic Normal Pressure Hydrocephalus, Japanese Society of Normal Pressure Hydrocephalus. Clinical guidelines for idiopathic normal pressure hydrocephalus. Neurol Med Chir (Tokyo). 2004;44(4): 222-3.

39. Stolze H, Kuhtz-Buschbeck JP, Drücke H, Jöhnk K, Diercks C, Palmié S, Mehdorn HM, Illert M, Deuschl G. Gait analysis in idiopathic normal pressure hydrocephalus-which parameters respond to the CSF tap test? Clin Neurophysiol. 2000;111(9):1678-86.

40. Stolze H, Kuhtz-Buschbeck JP, Drucke H, Johnk K, Illert M, Deuschl G. Comparative analysis of the gait disorder of normal pressure hydrocephalus and Parkinson's disease. J Neurol Neurosurg Psychiatry. 2001;70(3):289-97.

41. Knutsson E, Lying-Tunell $U$. Gait apraxia in normal-pressure hydrocephalus: patterns of movement and muscle activation. Neurology. 1985;35(2):155-60.

42. Zaaroor M, Bleich N, Chistyakov A, Pratt H, Feinsod M. Motor evoked potentials in the preoperative and postoperative assessment of normal pressure hydrocephalus. J Neurol Neurosurg Psychiatry. 1997;62(5):51721.

43. Calzada MD, Poca MA, Sahuquillo J, Matarin M, Mataro M, Solana E. Cognitive event-related bran potentials (P300) in patients with normal pressure hydrocephalus. Results of a prospective study. Neurologia. 2010;25:32-39.

44. Saito M, Nishio Y, Kanno S, Uchiyama M, Hayashi A, Takagi M, Kikuchi H, Yamasaki H, Shimomura T, lizuka O, Mori E. Cognitive profile of idiopathic normal pressure hydrocephalus. Dement Geriatr Cogn Dis Extra. 2011;1(1):202-211.

45. Iddon JL, Pickard JJL, Cross JJL, Griffiths PD, Czosnyka M, Sahakian BJ, Specific patterns of cognitive impairment in patients with idiopathic normal pressure hydrocephalus and Alzheimer's disease: a pilot study. J. Neurol Neurosurg Psychiatry. 1999;67(6):723-32.

46. Benejam B, Poca MA, Junque C, Solana E, Sahuquillo J. Alteraciones cognitivas em pacientes com hidrocefalia crónica del adulto ("normotensiva"). Propuesta de um protocolo para su evaluación clínica. Neurocirurgía. 2008; 19(4):309-21.

47. Bret P, Guyotat J, Chazal J. In normal pressure hydrocephalus a valid concept in 2002? A reappraisal in five questions and proposal for a new designation of the syndrome as "chronic hydrocephalus". J. Neurol Neurosurg Psychiatry. 2002;73(1):9-12. 
48. Takeuchi M, Hayashi N, Takaiwa A, Hamada H, Kuwayama N, Hirashima $\mathrm{Y}$, Matsui $\mathrm{M}$, Endo $\mathrm{S}$. Clinical value of constructional skill testing in patients with secondary normal pressure hydrocephalus. Neurol Med Chir (Tokyo). 2007;47(7):322-24.

49 Coll-Planas L, Denkinger MD, Nikolaus T. Relationship of urinary incontinence and late-life disability: implications for clinical work and research in geriatrics. Z Gerontol Geriatr. 2008;41(4):283-90.

50. Ahlberg J, Norlen L, Blomstrand C, Wikkelsö C. Outcome of shunt operation on urinary incontinence in normal pressure hydrocephalus predicted by lumbar puncture. $J$ Neurol Neurosurg Psychiatry. 1988;51(1):105-8.

51. Winter DA. Human balance and posture control during standing and walking. Gait \& Posture. 1995;3:193-214.

52. Toma AK, Tarnaris A, Neil D, Laurence D. Watkins LD. Working towards patient oriented outcome assessment in normal pressure hydrocephalus, what is the most important? Acta Neurochir (Wien). 2011;153(1):177-180.

53. Riberto $M$, Miyazaki $M H$, Jorge Filho $D$, Sakamoto $H$, Battistella LR. Reproductivity brazilian version of functional independence measure. Acta Fisiatr. $2001 ; 8(1): 45-52$.

54. Riberto M, Miyazaki MH, Jucá SSH, Sakamoto H, Pinto PPN, Battistella LR. Validation of the Brazilian version of functional independence measure. Acta Fisiatr. 2004;11(2):72-76.

55. Talmelli LFS, Gratão ACM, Kusumota L, Rodrigues RAP. Functional independence level and cognitive deficit in elderly individuals with Alzheimer's disease. Rev Esc Enferm USP. 2010;44(4): 932-8.

56. Yamada S, Liu M, Hase K, Tanaka N, Fujiwara T, Tsuji T, Ushiba J. Development of a short version of the motor fim for use in long-term care settings. J Rehabil Med. 2006;38(1):50-56.

57. Miyamoto ST, Lombardi Jr I, Gerg KO, Ramos LR, Natour J. Brazilian version of the Berg balance scale; Braz J Med Biol Res. 2004;37(9):141121.

58. Berg K, Maki B, Williams J, Holliday PJ, Wood-Dauphinee SL. Clinical and laboratory measures of postural balance in an elderly population. Arch Phys Med Rehabil. 1992;73(11):1073-80.

59. Shumway-Cook A, Gruber W, Baldwin M, Liao S. The effect of multidimensional exercises on balance, mobility, and fall risk in community-dwelling older adults. Phys Ther. 1997;77(1):46-57.

60. Castro SM, Perracini MR, Ganança FF. Versão brasileira do Dynamic Gait Index. Rev Bras Otorrinolaringol. 2006;72(6):817-25. 
61. Wrisley DM, Walker ML, Echternach JL, Strasnick B. Reliability of the dynamic gait index in people with vestibular disorders. Arch Phys Med Rehabil. 2003;84(10):1528-33.

62. Podsiadlo D, Richardson S. The timed 'Up \& Go": a test of basic functional mobility for frail elderly persons. J Am Geriatr Soc. 1991;39(2):142-8.

63. Bischoff HA, Stähelin HB, Monsch AU, Iversen MD, Weyh A, von Dechend M, Akos R, Conzelmann M, Dick W, Theiler R. Identifying a cut-off point for normal mobility: a comparison of the timed "up and go" test in community-dwelling and institutionalised elderly women. Age Ageing. 2003;32(3):315-20.

64. Kubo Y, Kazui H, Yoshida T, Kito Y, Kimura N, Tokunaga H, Ogino A, Miyake $\mathrm{H}$, Ishikawa $M$, Takeda $M$. Validation of grading scale for evaluating symptoms of Idiopathic Normal-Pressure Hydrocephalus. Dement Geriatr Cogn Disord. 2008;25(1):37-45.

65. Ishikawa M, Hashimoto M, Mori E, Kuwana N, Kazui $H$. The value of cerebrospinal fluid tap test for predicting shunt effectiveness in idiopathic normal pressure hydrocephalus. Fluids Barriers CNS. 2012;9(1):1.

66. Hellstrom $\mathrm{P}$, Klinge $\mathrm{P}$, Tans J, Wikkelso. A new scale for assessment of severity and outcome in iNPH. Acta Neurol Scand. 2012;126(4):229-37.

67. Guillemin F, Bombardier C, Beaton D. Cross-cultural adaptation of healthrelated quality of life measure: literature review and guidelines. J Clin Epidemiol. 1993;46(12):1417-32.

68. Hamilton BB, Laughlin JA, Fiedler RC, Granger CV. Interrater reliability of the 7-level functional independence measure (FIM). Scand J Rehabil Med. 1994;26(3):115-9.

69. Shumway-Cook A, Woollacott MH. Uma estrutura conceitual na prática clínica. In: Controle motor: teoria e aplicações práticas. São Paulo: Manole; 2003. p.103-18.

70. Shumway-Cook A, Brauer S, Woollacott M. Predicting the probability for falls in community-dwelling older adults Using The Timed Up \& Go Test. Phys Ther. 2000;80(9):896-903.

71. Loth EA, Albuquerque CE, Bertolini GRF. Avaliação do Timed Up and Go como preditor de quedas em uma amostra de idosas que relataram queda em 2003. Reabilitar. 2004;1:9-12.

72. Waltz CF, Sylvia BM. Accountability and outcome measurement: where do we go from here? Clin Nurse Spec. 1991;5(4):202-3.

73. Pasquali L. Psicometria: teoria e aplicações. Brasília: Editora UnB. 1997. 
74. Hashimoto M, Ishikawa M, Mori E, Nobumasa E, Kuwana N, Study of INPH on neurological improvement (SINPHONI). Diagnosis of idiopathic normal pressure hydrocephalus is supported by MRI-based scheme: a prospective cohort study. Cerebrospinal Fluid Res. 2010;31:7-18.

75. Tous NC, Cutillas AMF, Infante AMJ, Granados GO, Rojas ESJ, Velasco BL, Corral CS, Serrano MAR, Almansa AH. Hidrocefalia crónica del adulto: diagnóstico, tratamiento y evolución. Estudio prospectivo. Neurocirugía. 2013;24(3):93-101.

76. Owler BK, Momjian S, Czosnyka Z, Czosnyka M, Péna A, Harris NG, Smielewski P, Fryer T, Donovan T, Coles J, Carpenter A, Pickard JD. Normal pressure hydrocephalus and cerebral blood flow: a PET study of baseline values. J Cereb Blood Flow Metab. 2004;24(1):17-23.

77. Macêdo AML, Cerchiari EAN, Alvarenga MRM, Faccenda O, Oliveira MAC. Avaliação funcional de idosos com déficit cognitivo. Acta Paul Enferm. 2012;25(3):358-63. 
9 APÊNDICE 


\section{APÊNDICE}

RESEARCH-HUMAN-CLINICAL TRIALS

Fernando Campos Gomes

Pinto, PhD*

Felippe Saad, MD*

Matheus Fernandes de

Oliveira, MD $\ddagger$

Renan Muralho Pereiras

Fernanda Letkaske de

Miranda, MD*

Juliana Benevenuto Tornai,

MD*

Maria Izabel Romăo Lopes,

MD*

Eduardo Santamaria

Carvalhal Ribas, MD*

Emilia Aparecida Valinetti,

$\mathrm{PhD}^{*}$

Manoel Jacobsen Teixeira,

$\mathrm{PhD}^{*}$

"Division of Functional Neurosurgery of the Institute of Psychiatry, Hospital das Clinicas, Universidade de Sảo Paulo, Sáo Paulo, Brazil; tNeurosurgery Residency Program, Department of Neurosurgery. Hospital do Servidor Público Estadual de Sáo Paulo, Sáo Paulo, Brazili; śneurosurgery League, Faculty of Medicine, University of Sao Paulo and medical student at Universidade Anhembi-Morumbi, Sầo Paulo, Brazil

Correspondence:

Femando Campos Gomes Pinto, PhD,

Av Angélica, 1968, cj.21,

Săo Paulo, SP. Brazil,

CEP 01228-200.

E-mail: neurofernandogrgmail.com

Received, May 18, 2012

Accepted, December 18, 2012

Published Online, January 10, 2013

Copyright 12013 by the

Congress of Neurological Surgeons

\section{Role of Endoscopic Third Ventriculostomy and Ventriculoperitoneal Shunt in Idiopathic Normal Pressure Hydrocephalus: Preliminary Results of a Randomized Clinical Trial}

BACKGROUND: Currently, the most common treatment for idiopathic normal pressure hydrocephalus (INPH) is a ventriculoperitoneal shunt (VPS), generally with programmable valve implantation. Endoscopic third ventriculostomy (ETV) is another treatment option, and it does not require prosthesis implantation.

OBJECTIVE: To compare the functional neurological outcome in patients after 12 months of treatment with INPH by using 2 different techniques: ETV or VPS.

METHODS: Randomized, parallel, open-label trial involving the study of 42 patients with INPH and a positive response to the tap test, from January 2009 to January 2012. ETV was performed with a rigid endoscope with a $30^{\circ}$ lens (Minop, Aesculap), and VPS was performed with a fixed-pressure valve (PS Medical, Medtronic). The outcome was assessed 12 months after surgery. The neurological function outcomes were based on the results of 6 clinical scales: mini-mental, Berg balance, dynamic gait index, functional independence measure, timed up and go, and normal pressure hydrocephalus.

RESULTS: There was a statistically significant difference between the 2 groups after 12 months of follow-ups, and the VPS group showed better improvement results (ETV $=50 \%$, VPS $=76.9 \%)$

CONCLUSION: Compared with ETV, VPS is a superior method because it had better functional neurological outcomes 12 months after surgery.

KEY WORDS: Cerebrospinal fluid shunts, Endoscopic third ventriculostomy, Idiopathic normal pressure hydrocephalus

Neurosurgery 72.845-854, $2013 \quad$ DOL: 10.1227/NEU.0bo13e318285b37C www.neurosurgery-online.com

diopathic normal pressure hydrocephalus (INPH) is characterized by progressive gait apraxia, cognitive dysfunction, and urinary incontinence (Hakim-Adams syndrome). INPH is associated with communicating hydrocephalus (as demonstrated by brain computed tomography $[\mathrm{CT}]$ or magnetic resonance imaging [MRI]) and cerebrospinal fluid (CSF) pressure within the normal range $\left(7-24 \mathrm{~cm} \mathrm{H}_{2} \mathrm{O}\right)$. The clinical triad of

ABBREVIATIONS: BERG, Berg balance scale; DGI, dynamic gait index; EI, Evans index; ETV, endoscopic third ventriculostomy; FIM, functional independence measure; INPH, idiopathic normal pressure hydrocephalus; MMSE, Mini-Mental State Examination; NPH Scale, NPH Japanese Scale; TT, Exap test; TUG, timed ap test, TUG, timed up and go; VPS, ventriculoperitoneal shunt
Hakim-Adams syndrome is present in approximately $50 \%$ of cases; however, only 1 or a combination of 2 symptoms should be considered for diagnostic investigation. ${ }^{1-3}$

Currently, there are 2 main therapeutic options: ventriculoperitoneal shunt (VPS) and endoscopic third ventriculostomy (ETV). The most widely accepted and performed option is the VPS with programmable valve implantation. Nevertheless, it is widely accepted that surgical treatment of patients with INPH is necessary, because surgery has been associated with a positive impact on the course of the disease.

In 2008, an Italian multicenter study reported the benefits of ETV in the treatment of 110 patients with INPH. After a 24-month follow-up period, it was reported that $69.1 \%$ of the patients had improved. ${ }^{6}$ This therapeutic success rate is 
consistent with the results obtained with the VPS, ranging from $69 \%$ to $90 \% .^{7-13}$ However, that was a retrospective study, and the patients were not submitted to predictive functional tests, such as the tap test (TT), which is a test that is common in the literature. ${ }^{1 / 4}$

By performing a prospective randomized study, our objective is to compare the functional neurological outcomes of patients with INPH who have been treated with 2 different techniques: VPS or ETV.

\section{PATIENTS AND METHODS}

\section{Trial Design}

The study was a randomized clinical trial with a parallel design. The allocation ratio was 1:1. The parients were users of the medical facility and met the eligibility criteria

\section{Participants}

The adopted diagnostic criteria for probable INPH ${ }^{15}$ were as follows: a. Clinical: progressive clinical picture of gait apraxia, cognitive impairment (mainly recent memory and executive functions), and sphincter incontinence (urinary and/or fecal). The presence of only 1 or a combination of 2 symptoms is also seen as a suggestive criterion of the diagnosis. No personal history of subarachnoid hemorthage, head trauma, cranial neurosurgery for any reason, or central nervous system infection.

b. Radiological: ventricular dilation, confirmed by brain CT and MRI showing only the communicating hydrocephalus and an Evans index (EI) of $>30 \%$.

c. Manometric: CSF pressure within the normal range, as demonstrated by the opening pressure on lumbar CSF puncrure berween 7 and 24 $\mathrm{cm} \mathrm{H}_{2} \mathrm{O}$.

Inclusion criteria included the diagnosis of probable INPH, age 55 to 75 years, duration of symptoms $<24$ months, preserved ambulation even with 2 supports, absence of other dementia syndromes, absence of malignant disease, compensated clinical comorbidities (hypertension. diabetes mellitus, hormonal disorders), positive TT result, and free and informed consent signed by patients and family members.

Exclusion criteria included the diagnosis of secondary normal pressure hydrocephalus $(\mathrm{NPH})$, age $<55$ or $>75$ years, duration of symptom $>24$ months, other associated dementia syndromes, incapacity to walk, malignancy, uncontrolled clinical comorbidities, negative TT result, or refusal to participate in the study by a family member or the patient.

This is a prospective randomized study conducted from January 2009 to January 2012 in patients with INPH at the Institute of Psychiatry, Hospital das Clinicas, Faculdade de Medicina da Universidade de Sáo Paulo, after approval by the ethics committee (CAPPESQ 0348/09).

\section{Interventions}

The TT was performed in the preoperative period to determine the CSF pressure and therapeutic prognosis by withdrawing $40 \mathrm{~mL}$ of CSF. ${ }^{16,17}$ Clinical evaluation was performed by a multidisciplinary team consisting of a neurosurgeon, a neurologist, and a physiotherapist. Two pre-TT evaluations were performed with a 1-week interval. Two additional evaluations were performed 3 and 72 hours after lumbar puncture. Each evaluation consisted of 6 clinical scales, and the TT was considered positive when the patient scored at least 2 points higher on the NPH Japanese Scale. The other scales were considered to be secondary outcomes, and, in general, they were related to changes in the NPH Japanese Scale. The best result of the 2 pre-TT evaluations and the 2 post-TT assessments was taken into account for comparison.

\section{Scales Used}

1. Mini-Mental Status Examination ${ }^{18,19}$ (MMSE)

Objective: to evaluate cognitive alterations

Score: 0 to 30 (higher is better).

2. The Berg Balance Scale ${ }^{20,21}$ (BERG)

Objective: to evaluate the functional balance capacity in the following positions: sitting, standing, and leg support.

Score: 0 to 56 (higher is better).

3. Functional Independence Measure ${ }^{22}$ (FIM)

Objective: to assess the degree of dependence for the functional activities of daily living.

Score: 18 to 126 (higher is better; 18-36 total dependence, 37-90 moderate dependence, and 91-126 independent).

4. Dynamic Gait Index ${ }^{23}$ (DGI)

Objective: to assess the disorders of gait dynamics. The individual is submitted to a walking test that consists of walking $6 \mathrm{~m}$ while making acceleration and deceleration movements and going through obstacles.

Score: 0 to 24 (higher is better).

5. NPH Japanese Scale ${ }^{9}$ (NPH Scale)

Objective: to score the patients according to the clinical characteristics of the NPH triad.

Score: 0 to 12 (higher is worse).

Gait Disorder

0 Absent

1 Unstable gait, but independent

2 Walks with 1 support

3 Walks with 2 supports or a walker

4 Cannot walk

Dementia

0 Absent

1 No apparent dementia, but apathetic

2 Socially dependent, but independent at home

3 Partially dependent at home

4 Totally dependent

Urinary Incontinence

0 Absent

1 Absent, but has polyuria or urinary urgency

2 Sometimes, only at night

3 Sometimes, even during the day

4 Frequent

6. Timed Up and Go ${ }^{24}$ (TUG)

Objective: to assess mobility and balance. The test quantifies functional mobility in seconds by using the time during which the individual performs a task. The time it takes for the individual to rise from the chair, walk for $3 \mathrm{~m}$ up to a predetermined point, return to the chair, and sit down is measured.

Normal performance for healthy adults: 10 to 12 seconds. 
Normal for impaired adults: 12.01 to 20 seconds.

Functional impairment: $>20.01$ seconds.

ETV was performed via a right precoronal burr hole (Kocher point) with a rigid ventricular neuroendoscope containing a $30^{\circ}$ lens (Minop, Aesculap). The floor of the third ventricle was bluntly perforated in the midline halfway between the mammillary bodies and the infundibular recess. The fenestration was subsequently enlarged by inflating the balloon of a 4 F Fogarty catheter. The ventriculostomy size was approximately 4 to $6 \mathrm{~mm}$.

VPS was performed via a right precoronal burr hole (Kocher point). The chosen valve pressure (PS Medical, Medtronic) was based on the final manometry value at the TT. After the removal of $40 \mathrm{~mL}$, a final pressure of $<4 \mathrm{~cm} \mathrm{H} \mathrm{H}_{2} \mathrm{O}$ resulted in the selection of a low-pressure valve; a final pressure between 4 and $10 \mathrm{~cm} \mathrm{H}_{2} \mathrm{O}$ resulted in the selection of a medium-pressure valve; and a final pressure $>10 \mathrm{~cm} \mathrm{H}_{2} \mathrm{O}$ resulted in the selection of a high-pressure valve.

\section{Outcomes}

Our hypothesis is that INPH treatment with the VPS is a superior option in comparison with ETV. To test this hypothesis, a complete evaluation was performed with the validated scales to quantify and compare the clinical profiles before and after surgery. The BERG Scale, DGI, FIM, MMSE, NPH Scale, and TUG were compared between the 2 groups. All patients were followed for 12 months, with prescheduled consultations at 3, 6, and 12 months after surgery. The patients were evaluated according to the 6 scales at 3 and 12 months.

\section{Primary Outcome}

After 1 year, the late postoperative result was classified as positive if the patient had at least a 2 points higher score on the NPH Scale.

\section{Secondary Outcomes}

The other scales, which were generally related to changes in the NPH Scale, were considered secondary outcomes. Surgical complications were also quantified in both groups to analyze safery and efficiency profiles.

\section{Sample Size}

The sample size was calculated according to a specific formula. The Altman normogram was performed to test the difference between the means in the BERG Scale ${ }^{25}$ (before and after surgery) in both treatment groups. Assuming that variances were equal in both groups, the difference between means was given as $85 \%$ (taking into consideration the differences in BERG) of the standard deviation, which contained a significance level of $5 \%$ and a power of $80 \%$. The minimum sample size was 22 patients for each group. Because $10 \%$ of the patients did not perform follow-ups, each group comprised 25 patients, Randomization was performed in blocks of 10 parients to ensure that equal numbers of participants were in each group. ${ }^{26}$

In our sample, 21 patients were randomly assigned to the ETV group and 21 were randomly assigned to the VPS group, totaling 42 patients. This number of patients is clearly less than the hypothetical number of patients, which is 50 .

\section{Randomization}

The assignment of participants was performed in the operating room after the patients had been anesthetized. An independent physician from the surgical ward of the hospital randomly chose between 2 equally sized and opaque white sealed envelopes that were placed side to side over a table. Each envelope contained a white sheet of paper with the name of a procedure on it (either VPS or ETV), thus choosing the intervention to be performed. All of the material needed for both procedures was present in the operating room. At this point, there was no blinding.

The patients were divided into 2 groups: group 1, ETV, and group 2, VPS.

Complications such as CSF leak, infection, shunt malfunction, or hyperdrainage were strictly observed, treated, and recorded for the later comparisons between the groups.

In the immediate postoperative period, and at 6 months postoperation, as well, all of the patients underwent a brain CT. An MRI with CSF flow study was performed on patients who underwent ETV but did not show clinical improvement for 3 months. This procedure was performed to determine the parency of the orifice on the third ventricle floor. The patient then underwent a VPS with a fixed-pressure valve. These patients were considered ETV failures. The results after the second procedure (VPS) were not used for statistical calculations, because we strongly believe that a patient who undergoes VPS after an unsuccessful previous ETV has a very different clinical profile than a patient who has not undergone ETV. Because of hydrodynamic alterations, we believe that these patients represent 2 different groups.

\section{Statistical Methods}

In this study, numerical data are presented as the mean with the range. Categorical data are presented as percentages. To determine the distribution of our data, the Kolmogorov-Smirnov test was performed. Statistical analysis of the clinical results 12 months after surgery was performed by using the Student $t$ test for the paired and unpaired groups. The significance level was established as $P<.05$. All tests were corrected for multiple comparisons.

After 1 year, the late postoperative result was classified as positive when the patient scored at least 2 points higher on the NPH Scalc. The other scales, which were generally related to changes in the NPH Scale, were considered to be secondary outcomes.

All of the scales were directly compared. However, if a particular scale was limited for a specific symptom of INPH, then the NPH Scale was considered the main outcome after it had been validated and designed to evaluate each of the 3 elements of the classic triad. Thus, improvement or an unchanged/worse outcome was based on the NPH Scale.

\section{RESULTS}

\section{Participant Flow}

Of the 90 patients who were diagnosed with NPH and treated during this period, 48 were excluded, because they did not meet the inclusion criteria for this study. Forty-two patients were enrolled. The full participant flow is shown in Figure 1.

\section{Baseline Data}

Of these patients, 24 were men and 18 were women. In the VPS group, ages ranged from 60 to 75 years (mean, 70 years). In the ETV group, the mean age was 71 years, ranging from 62 to 73 years. There was no statistically significant difference between the ages and the sex distribution of the VPS and ETV groups $(P>.05)$.

Twenty-one patients had the complete NPH clinical triad, 10 patients had gait apraxia and cognitive impairment, 4 patients had 


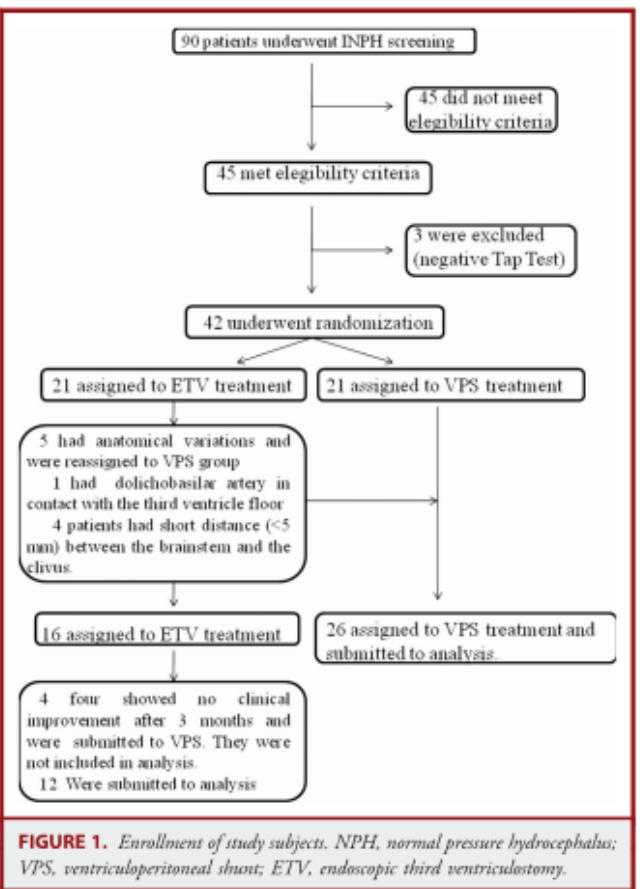

gait apraxia and urinary incontinence, and 7 patients only had gait apraxia. All of the patients had communicating hydrocephalus according to CT and MRI, and the EI ranged from $30 \%$ to $50.6 \%$ (mean, 38.9\%).

At TT, the initial pressure ranged from 20 to $12 \mathrm{~cm} \mathrm{H}_{2} \mathrm{O}$ (mean, 15), and the final pressure after the withdrawal of $40 \mathrm{~mL}$ ranged from 12 to $2 \mathrm{~cm} \mathrm{H} \mathrm{H}_{2} \mathrm{O}$ (mean, 6). The results of chemocytological analysis were normal in all samples and showed no reaction for syphilis, tuberculosis, cryptococcosis, or neurocysticercosis.

Table 1 shows the values of the scores on the 6 scales before and after the TT and the postoperative follow-up period (3-12 months).

\section{Group 1: ETV}

Outcomes

Of the 21 patients randomly assigned to the ETV group, 5 had anatomical features that made the endoscopic procedure dangerous. One parient had a dolicho basilar artery that was in contact with the third ventricle floor, and 4 patients had a short distance $(<5 \mathrm{~mm})$ between the brainstem and the clivus. These 5 patients were relocated to group 2 to avoid basilar artery injury.
Of the 16 patients who constituted group 1,9 were men and 7 were women. The mean age was 70 years, ranging from 60 to 75 years. The initial pressure ranged from 18 to $10 \mathrm{~cm} \mathrm{H}_{2} \mathrm{O}$ (mean, 15) before the TT, and the final pressure after the withdrawal of $40 \mathrm{~mL}$ ranged from 12 to $3 \mathrm{~cm} \mathrm{H}_{2} \mathrm{O}$ (mean, 6).

Of the patients undergoing the ETV, 4 showed no clinical improvement after 3 months. These patients underwent an MRI, which showed that they had a patent orifice in the third ventricle floor. The patients then underwent the VPS, but only $2(50 \%)$ showed improvement after 1 year of follow-up. The other 12 patients $(12 / 16,75 \%)$ improved at 3 months post-ETV, but this improvement was only partially maintained after 12 months.

In the 12 patients who had some degree of clinical improvement, oscillatory up and down movements were observed on the third ventricle floor in the intraoperative period immediately after ETV ("flag signal"). The 4 patients who did not improve showed no "flag signal." A typical subject from the ETV group is shown in Figure 2.

\section{Harms}

There were no intraoperative or postoperative complications, such as bleeding, fornix injury, infection, hematoma, or CSF leaks.

No patient in the ETV group showed a significant reduction in ventricular size on the brain CT. The EI ranged from $31 \%$ to $50.6 \%$ (mean, $39.7 \%$ ) preoperatively and $31 \%$ to $49.1 \%$ (mean, $39.1 \%) 6$ months after ETV.

\section{Group 2: VPS}

\section{Outcomes}

Of the 21 patients randomly assigned to the VPS group and the 5 patients relocated from group 1,15 were men and 11 were women. The mean age was 71 years, ranging from 62 to 73 years. Seventeen low-pressure valves and 9 medium-pressure valves were initially implanted (PS Medical, Medtronic).

During the TT, the initial pressure ranged from 20 to $12 \mathrm{~cm}$ $\mathrm{H}_{2} \mathrm{O}$ (mean, 16), and the final pressure after the withdrawal of 40 $\mathrm{mL}$ ranged from 10 to $2 \mathrm{~cm} \mathrm{H} \mathrm{H}_{2} \mathrm{O}$ (mean, 6). A typical subject from the VPS group is shown in Figure 3.

Harms

In the VPS group, 5 of the patients $(5 / 26,19 \%)$ who underwent implantation of a low-pressure valve had overdrainage with a significant reduction in ventricular size and a chronic subdural hematoma. These patients underwent another operation during which hematoma drainage was performed and the valve was replaced with a medium-pressure valve (PS Medical, Medtronic).

After the 3-and 12-month follow-up periods, 20 patients (20/ $26,77 \%)$ showed improvement. No patient showed signs of infection.

In the remaining patients, ventricular reduction on the skull CT at 6 months was slight but evident. In these patients, the EI ranged from $30 \%$ to $48.1 \%$ (mean, $38.1 \%$ ) preoperatively, and $26.6 \%$ to $41.4 \%$ (mean, $36.6 \%$ ) 6 months after VPS. 


\begin{tabular}{|c|c|c|c|c|}
\hline Group & Best Before TT & Best After TT & After 3 Months & After 12 Months \\
\hline \multicolumn{5}{|c|}{ Group 1-ETV $(n=16)$} \\
\hline MMSE & $21(29-8)$ & $21(30-12)$ & $22(28-3)$ & $22(28-9)$ \\
\hline BERG & $28(51-1)$ & $35(56-1)$ & $31(49-0)$ & $29(49-0)$ \\
\hline FIM & $79(115-22)$ & $85(116-32)$ & $78(108-21)$ & $82(110-22)$ \\
\hline DGI & $9(17-1)$ & $13(24-1)$ & $13(23-0)$ & $8(20-0)$ \\
\hline NPH Scale & $7(11-2)$ & $6(10-2)$ & $6(12-2)$ & $6(12-3)$ \\
\hline TUG & $47(158-10)$ & $34(110-9)$ & $33(105-12)$ & $46(95-12)$ \\
\hline \multicolumn{5}{|c|}{ Group 2-VPS $(n=26)$} \\
\hline MMSE & $21(29-9)$ & $22(30-11)$ & $20(27-12)$ & $20(30-10)$ \\
\hline BERG & $27(51-3)$ & $39(55-3)$ & $39(56-5)$ & $37(56-5)$ \\
\hline FIM & $76(111-19)$ & $85(118-44)$ & 91 (126-49) & $92(124-49)$ \\
\hline DGI & $10(20-1)$ & $15(24-5)$ & $15(24-0)$ & $14(24-0)$ \\
\hline NPH Scale & $6(11-1)$ & $5(11-1)$ & $5(12-0)$ & $4(12-0)$ \\
\hline TUG & $42(156-10)$ & $25(60-7)$ & $29(76-7)$ & $32(110-7)$ \\
\hline
\end{tabular}

"TT, tap test; ETV, endoscopic third ventriculostomy; VPS, ventriculoperitoneal shunt; MMSE, mini-mental state examination; BERG, Berg balance scale; FIM, functional independence measure; DGl, dynamic gait index; NPH Scale, NPH Japanese scale; TUG, timed up and go.

balues shown are the average (max-min).

\section{Comparison Between the Groups}

The ETV group had slightly better scores on some of the scales (MMSE, BERG, NPH Scale, and TUG) after 3 months, but this improvement was only partially maintained after 12 months. Some of the scales had lower scores (BERG, DGI), whereas the TUG returned to the pre-TT values (Table 1).

The VPS group showed better scores on the motor scales after 3 and 12 months (BERG, FIM, DGI, NPH Scale, and TUG). In addition to maintaining the improvement achieved at 3 months, the patients in these groups had increased scores on 2 additional scales (FIM and NPH Scale). For the rest of the scales, the variations were not significant and were considered stable. The TUG test score was considered better than the pre-TT value (Table 1).

The only surgical complication observed in the follow-up that was present in the VPS group was a subdural hematoma, which happened in 5 of 26 patients. These patients, however, underwent a successful valve replacement operation and had a favorable 12-month outcome (Table 2).

The results were analyzed by the Student $t$ test, showing that there was a statistically significant difference between the percentages of patients who improved in both groups after the 12-month follow-up (Table 3). These results suggest that VPS treatment results in a more favorable outcome. In contrast, the results from the ETV group did not show any differences between the initial pre-TT tests and the 12-month follow-up (Table 1).

\section{DISCUSSION}

\section{Limitations}

Initially, the TT was the only preoperative test used for the treatment of patients because of its applicability, validity and availability, as well as patient comfort, because hospitalization was not required during the protocol. Additionally, we believe that this procedure did not lead to significant issues for the randomization process of the clinical trial.

However, the TT has a limited sensitivity $(26 \%-61 \%)$ in comparison with the infusion test $(57 \%-100 \%)$ and results in a prolonged external lumbar drainage in excess of $300 \mathrm{~mL}(50 \%-$ $100 \%$ ), which may cause missing of potential patients. In terms of the positive predictive value, the TT is similar to the infusion test $(75 \%-92 \%)$, and both tests are below the external lumbar drainage $(80 \%-100 \%) .{ }^{14,16}$

In addition, the MMSE has significant limitations, and cognitive questionnaires that are more complete, such as the Montreal Cognitive Assessment and the Cambridge Cognitive Examination, are available. ${ }^{27,28}$ However, the MMSE test is a general tool used worldwide to assess dementia syndromes and is widely used in the literature.

Interpretation of the results should be made cautiously. Because gait apraxia was the only clinical outcome with a significant difference between treatment groups, there may be a potential diagnosis bias because of the limited values of the MMSE. The use of

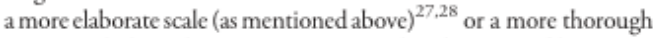
neuropsychological assessment may resolve the issue of bias.

An important limitation of this trial is that the current management of INPH is the implantation of a programmable VPS. Therefore, the use of nonprogrammable valves may decrease, but not invalidate, the quality of the data presented by the study. We did not use the programmable valve prosthesis in our patients because it is not available for use in the Brazilian public health system. In addition, almost $20 \%$ of the patients in the VPS group experienced complications, which included subdural hematomas in the implanted low-pressure valves, that required reoperation. 
PINTO ET AL

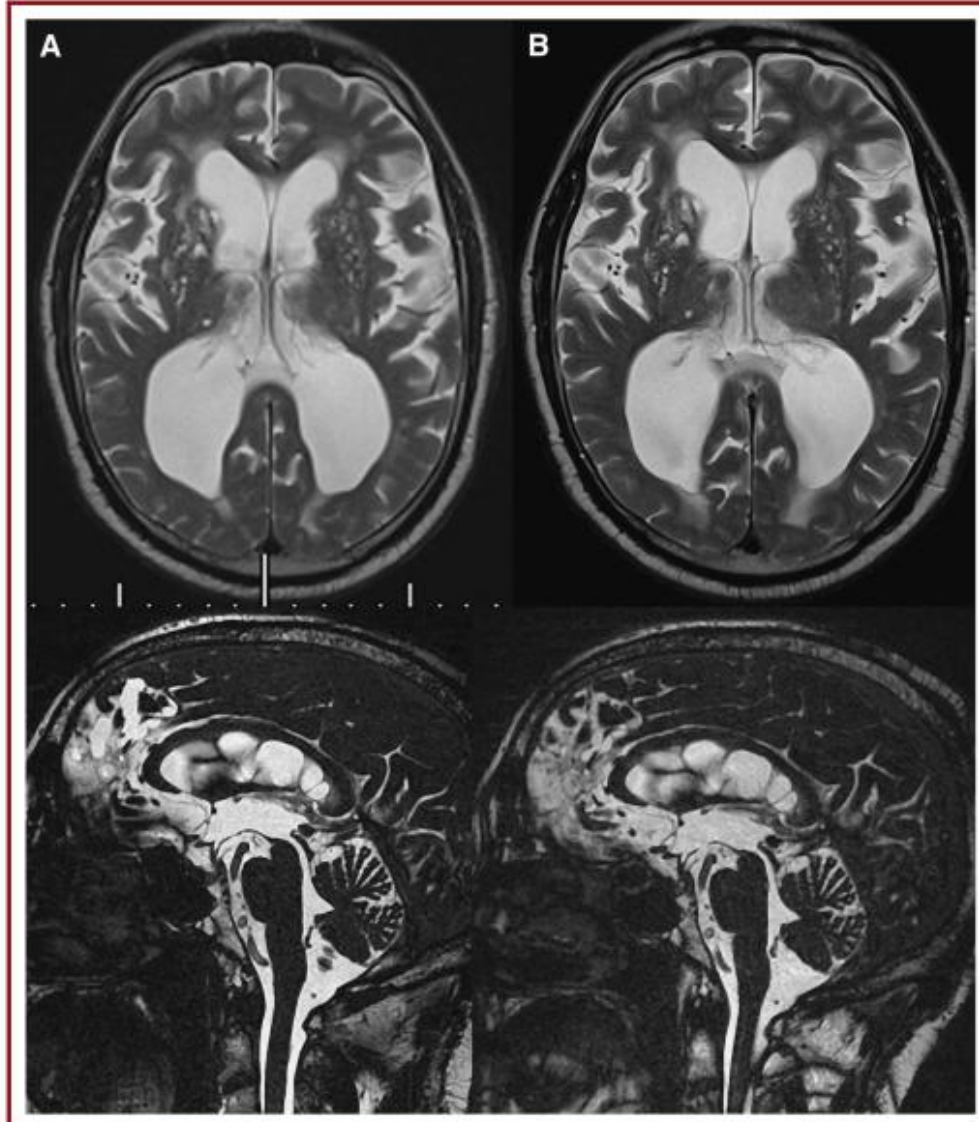

FIGURE 2. A sypical sabject from the ETV group. A 75-yar-old man presnting the INPH clinical triad for 15 months before surgery. A, axial and sagittal MRI images before ETV, revedling an Evans ratio of approximately 0.35. B, axial and sagittal MRI images affer ETV, revealing a previous thivd ventriculostomy above the basilar artery. The Eusws natio remained the same. INPH. idiopadbic normal pressure bydrocephalus ETV, endosopic third vensriculostomy.

Although these complications could have been avoided with programmable valves, the final outcome of the results likely would have remained unchanged.

A larger sample follow-up might provide more strength to the conclusions exposed. However, this study highlights the controversy concerning INPH treatment and may generate discussion about future treatment options.

The discrepancies between the results for patients less than 65 years of age compared with those over 65 years of age are intriguing. It would be useful to compare the CT or MRI images of these 2 groups of patients to determine whether the hydrocephalus pattern is different, because these results would contribute to the understanding of the physiopathological differences between age groups as well as between different treatment options. This knowledge is important in determining which patients would benefit most from INPH treatment. Because this topic is beyond the scope of this article, we hope to address these questions in future scientific communications. 


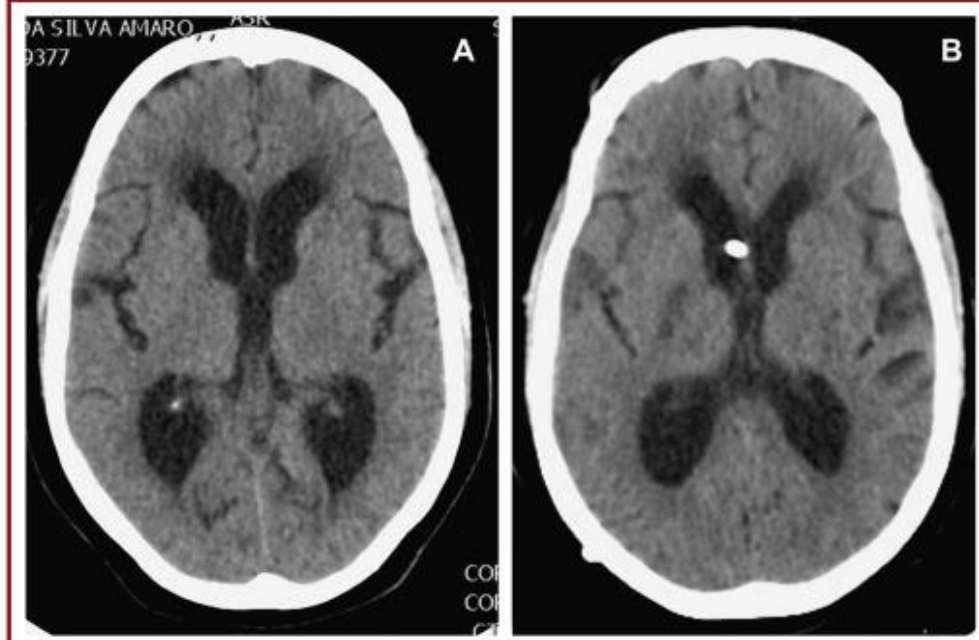

FIGURE 3. A sypical subject from the VPS group. A 68-year-old man presenting the INPH dinical triad for 12 months bofore surgery. A, avial CT images before VPS reveading an Evanu ratio of approximatcly 0.34. B, axtal CT images affer VPS recouling an Evans vatio of 0.33. INPH, idiopathic normal pressece lydracephalas. VPS, wentriculoperiteneal shunt.

Several confounding factors must be considered. Several medical diseases present with the same symptoms as the classical triad of NPH, and we are unable to entirely control for all of those diseases. For example, a large number of patients with dementialike syndromes have been categorized as having Alzheimer disease, $, 18,20$ and symptoms that are not typical to Alzheimer disease may be present in such patients.

Vascular dementia and other types of dementia are more frequent in older patients, which is also the case with NPH. Clinical comorbidities, such as hypertension and hypercholesterolemia, are also common features of different conditions and might interfere with the diagnosis of patients. Gait and urinary disturbances are also associated with a myriad of other diseases,

TABLE 2. Comparison of Complications Between Both Groups ${ }^{a, b}$

\begin{tabular}{|lcc|}
\hline Complication & ETV Group & VPS Group \\
\hline Significant bleeding & 0 & 0 \\
Fornix injury & 0 & 0 \\
Subdural hematoma & 0 & $5 / 26(19 \%)$ \\
Infection, wound dehiscence, and CSF leaks & 0 & 0 \\
\hline
\end{tabular}

ETV, endoscopic third ventriculostomy: VPS, ventriculoperitoneal shunt. The only complication observed in the follow-up was present in the VPS group and was a subdural hematoma. Its frequency was high (19:6), although with a successful reoperation, valve replacement, and favorable 12-month outcome. such as Parkinson disease and urologic/gynecologic/psychiatric urinary incontinence. Rigid patient selection criteria, as described in Methods, were adopted to address these issues. Therefore, we do not believe that any confounding factors interfered with our analysis. For example, rather than identifying patients as having NPH gait symptoms, we referred to these patients as being unable to walk because of orthopedic diseases.

\section{Generalizability}

The identification of patients with INPH and the implementation of effective treatment for INPH are current challenges for physicians and neurosurgeons. INPH is a disorder classified as a type of dementia that affects elderly persons and can be reversed if promptly diagnosed and treated.

With improvements in quality of life and the subsequent increase in life expectancy, it is expected that a greater number of elderly individuals will have this disease. In 2008 , the incidence and prevalence of INPH were calculated in a stable community of 220000 inhabitants in Norway, with values of 5.5/100 000 and $22 / 1000000$, respectively. ${ }^{29}$

The current recommended treatment is the implantation of VPS with a programmable valve. However, this type of treatment presents both the inconvenience of a prosthesis implant and medium- to long-term risk of complications requiring reoperation, such as infection, mechanical malfunction, and overdrainage. The UK registry shows a VPS reoperation rate of $22 \%$ in 5 years for the 
TABLE 3. A Statistically Significant Difference Between the Percentage of Patients Who Improved in Both Groups (ETV or VPS) 1 Year After Surgery $(\boldsymbol{P}<.05)^{a, b}$

\begin{tabular}{|lcccc|}
\hline Improvement (12 mo After Surgery) & ETV, $\mathbf{n}(\%)$ & VPS, $\mathbf{n}(\%)$ & Total, $\mathbf{n}(\%)$ & VPS After ETV Failure, $\mathbf{n}(\%)$ \\
\hline Yes & $8(50)$ & $20(76.9)$ & $28(66.7)$ & $2(50)$ \\
No & $8(50)$ & $6(23.1)$ & $14(33.3)$ & $2(50)$ \\
Total & $16(100)$ & $26(100)$ & $42(100)$ & $4(100)$
\end{tabular}

DTV, endoscopic third ventriculostomy: VPS, ventriculoperitoneal shunt.

'The patients that were submitted to VPS after ETV failure are shown in the last column.

treatment of hydrocephalus in adults. ${ }^{30}$ The siphoning effect, which is responsible for overdrainage, can be partially controlled with an antisiphoning device associated with the valve system, a flow-regulated valve, or a change in the programmable valveopening pressure so that overdrainage indications can be detected (subdural fluid collections). ${ }^{31}$

Thus, if INPH patients could be treated by ETV with the same long-term effects as the VPS, the risk of long-term complications would be reduced. The reduction of long-term complications would be an advantage for elderly patients, who often have comorbidities.

The Italian multicenter retrospective study published in 2008 caused great debate in the neurosurgical community. This study showed a success rate of $69.1 \%$ for ETV treatment in 110 patients with INPH after a follow-up period of at least 2 years. Much of the criticism of this work originated for the following reasons: there was a lack of a clear distinction between the cases of idiopathic $\mathrm{NPH}$ and possible cases of secondary NPH, and the predictive functional test used was the monitoring of intracranial pressure, rather than the tests that are more widely used in the literature, such as the TT, the lumbar infusion test, and external lumbar drainage monitoring for 72 hours. 6

\section{Interpretation}

To address unresolved issues in the literature, we improved the methodology commonly used and only included the patients with INPH that had a positive response to the TT and a preserved walking capacity. The design of this study was prospective, and the surgical treatment was randomized in the operating room immediately after the induction of anesthesia. The initial objective was to compare the functional neurological outcomes of patients with INPH within 1 year after either the VPS with a fixed pressure valve or ETV. In this study, we did not use the programmable valve prosthesis, because it was not available for use in the Brazilian public health system.

The TT was shown to be a universal predictive test. However, there were different results in the ETV and VPS groups. We observed that the percentage of patients who improved in the first year of follow-up was different in both groups, with values similar to those found in the literature for the VPS, which range from $70 \%$ to $90 \%$. $^{7-13}$ However, patients treated with the VPS showed a much more significant neurological functional gait improvement after 12 months than patients treated with ETV.

Five patients treated with the VPS $(5 / 26,19 \%)$ showed overdrainage with a significant reduction in ventricular size and chronic subdural hematoma. These patients underwent reoperation with hematoma drainage and valve replacement. We believe that these complications could have been avoided by using a programmable valve.

The patients who did not initially improve with ETV had the VPS implanted, and $50 \%$ of them improved after the second surgery, as previously mentioned in the literature. These patients had no pulse on the third ventricle floor ("flag signal") immediately after ETV. This finding was also described by Gangemi et al ${ }^{6}$ and represents the most important piece of information that is predictive of the therapeutic success of ETV. The results after the second procedure (VPS) were not used for the statistical calculations because we strongly believe that a patient with a previous ETV who is then submitted to a VPS has a very different clinical profile compared with a patient with no previous ETV.

We hypothesize that ETV promotes functional improvement in patients with INPH. We believe that during systole, when there is peak intracranial pressure, the surgically opened third ventricle floor will function as an escape mechanism for this mechanical energy, and, therefore, the frontal lobes, basal ganglia, and thalami will receive a lower intensity of the pressure pulse. This will lead to an improvement in blood perfusion in these regions, enabling the functional recovery of neurons affected by the progression of disease (INPH), ${ }^{4,32}$

Considering the preliminary results of the first year of follow-ups and the absence of the "flag signal" in all of the patients who underwent an ETV but did not improve, we suggest that if the "flag signal" during ETV treatment is not observed intraoperatively, the patient should immediately undergo VPS through the same burr to avoid a future surgical anesthetic procedure.

To assess the long-term effects of ETV and VPS (5 years), patients in this study remain under surveillance and monitoring. The other patients who have recently been included in the study but who have not yet completed the follow-up period for observation will increase the size of our cohort for future considerations.

Considering what has been observed thus far, we cannot suggest ETV as the best option for initial treatment of INPH, because the 
VPS had better functional neurological outcomes after 1 year. However, ETV can be considered a treatment option for INPH. ETV caused no significant improvement, but the scores on the scales were maintained. Having an untreated control group would indicate whether there was any impact on the natural evolution of INPH.

\section{CONCLUSION}

ETV and the VPS with fixed pressure represent different tools to manage INPH, and VPS shows the best gait outcome 12 months after surgery.

Future multicenter studies with larger sample sizes are expected to confirm our findings and address any issues about INPH surgical treatment.

\section{Disclosures}

The full trial protocol is discussed in the text. Further protocol data are available from the authors.

Trial Registration: CAPPESQ 0348/09. This study was carried out at the Hospital das Clínicas, University of Sáo Paulo, Säo Paulo, Brazil. There was ethical adherence, and the study was registered with the protocol CAPPESQ 0348/09. The authors have no personal financial or institutional interest in any of the drugs, materials, or devices described in this article.

\section{REFERENCES}

1. Adams RD, Fisher CM, Hakim S, Ojemann RG, Sweet WH. Sympromatic occult hydrocephalus with "normal" cerebrospinal-fluid pressure. A treatable syndrome N Engl J Med 1965;273:117-126.

2. Hakim CA, Hakim R, Hakim S. Normal-pressure hydrocephalus. Neurasugy Clin NAm. 2001;12(4):761-773, ix

3. Hakim S, Adams RD. The special dinical problem of sympeomatic hydrocephalus with normal cerebrospinal fluid pressure. Observations on cerebrospinal fluid hydrodynamics. J Neurol Sci. 1965:2(4):307-327.

4. de Oliveira MF, Pinto FC, Nishikuni K, Botelho RV, Lima AM, Rotta JM. Revisiting hydrocephalus as a model to study brain resilience. Front Hum Neurswi. 2011:5:181.

5. Toma AK, Stapleton S, Papadopoulos MC, Kitchen ND, Warkins LD. Narural history of idiopathic normal-pressure hydrocephalus. Nenrossory Rev. 2011:34(4): 433-439.

6. Gangemi M, Maiuri F, Naddeo M, et al. Endascopic third ventriculostomy in idiopanhic normal pressure hydrocephalus: an Italian multicenter study. Newroswergor. 2008;63(1):62-67; discassion 67-69

7. Boon AJ, Tans JT, Delwel EJ, et al. Dutch Normal-Pressure Hydrocephalus Srudy: randomized comparison of low- and medium-pressure shunts. / Neseorurg. 1998:88(3):490-495.

8. MoGirt MJ. Woodworth G, Coon AL, Thomas G, Williams MA, Rigamonti D. MoGirt MJ. Woodworth G, Coon AL, Thomas G, Willams MA, Rigamonti D.
Diagnosis, treatment, and analysis of long-term outcomes in idioparthic normalpressure hydrocephalus. Neurosurgery. 2008;62 (suppl 2):670-677.

9. Mori K. Management of idiopathic normal-pressure hydrocephalus: a multiinstitutional study conducted in Japan. J Nesowsurg. 2001;95(6):970-973.

10. Petersen RC, Mokri B, Laws ER Jr. Surgical treatment of idiopathic hydrocephalus in elderly paticnts. Nesowlogy. 1985;35(3):307-311.

11. Vanneste J, Augustijn P, Dirven C, Tan WF, Goedhart ZD. Shunting normalpressure hydrocephalus: do the benefits outweigh the risks? A multicenter study and literature review. Neworlogy. 1992:42(1):54-59.

12. Weiner $\mathrm{HL}$., Constantini S, Cohen $\mathrm{H}$, Wisoff JH. Current treatment of normalpressure hydrocephalus: comparison of flow-regulated and differential-pressure shunt valves. Neuresurgery. 1995:37(5):877-884.

13. Zemack G, Romner B. Adjustable valves in normal-pressure hydrocephalus: a recrospective study of 218 parients. Newrossargery. 2008;62(suppl 2):677-687.
14. Marmarou A, Bergsneider M, Klinge P, Relkin N, Black PM. The value of supplemental prognostic tests for the preoperative assessment of idiopathic normalpressure hydrocephalus. Newreswngery. 2005;57(suppl 3):S17-\$28; discussion ii-v.

15. Relkin N, Marmarou A, Klinge P, Bergsneider M, Black PM. Diagnosing idiopathic normal-pressure hydrocephalus. Nesrossogeren. 2005:57(suppl 3):S4S16; discussion it-v.

16. Wikkelsō C, Andersson H, Blomstrand C, Lindqvist G, Svendsen P. Normul pressure hydrocephalus. Predictive value of the cerebrospinal fluid tap-test. Actas Newrol Seand 1986;73(6):566-573.

17. Wikkelse C, Andersson H, Blomstrand C, Lindquist G. The clinical effect of lumbar puncture in normal pressure hydrocephalus. / Nearol Neurossogy $P_{\text {Pyodiatry. }}$ lumbar puncture in

18. Brucki SM, Nitrini R, Caramelli P, Bertolucci PH, Okamoto IH. Suggestions for urilization of the mini-mental state examination in Brazil [in Portuguese]. Arq Newropsigusistr. 2003;61(3B):777-781.

19. Folstein MF, Folstein SE, McHugh PR. "Mini-mental state". A practical method for grading the cognitive state of paticnts for the clinician. J Psydhiarr Ros. 1975;12 (3): $189-198$.

20. Berg KO, Wood-Dauphinee SL, Williams JI, Maki B. Measuring balance in the elderly: validation of an instrument. Can / Public Healsh, 1992:83(suppl 2): 57-\$11

21. Miyamoro ST, Lombardi Junior 1, Berg KO, Ramos LR, Narour J. Brazilian version of the Berg balance scale. Braz / Med Biol Res. 2004:37(9):1411-1421,

22. Desrosiers J. Rochette A, Noreau L. Bravo G. Hébert R, Boutin C. Comparison of two functional independence scales with a participation measure in post-stroke two functional independence scales with a participation m

23. De Castro SM, Perracini MR, Ganança FF. Dynamic Gait Index-Brazilian version. Braz J Otorhinolaryngol. 2006; $72(6): 817-825$

24. Podsiadlo D, Richardson S. The timed "Up \& Go": a test of basic functional mobility for frail elderly persons. J Am Geriatr Soc. 1991:39(2):142-148.

25. Rosner B. Fundamentals of Biestatistics. Belmont, CA: Thomson Brooks; 2006: 868 p.

26. Friedman LM, Furberg CD, DeMets DL. Fundinnentals of Clinical Trials. New York, NY: Springer-Verlag; 1998:361p

27. Brean A, Eide PK. Prevalence of probable idiopathic normal pressure hydrocephalus in a Norwegian population. Acta Neserol Scand. 2008;118(1):48-53.

28. $O^{\prime}$ Kane MC, Richards H. Winfield P, Pickard JD. The Unired Kingdom Shunt Registry. Ear J Pediatr Surg. 1997; 7(suppl 1):56.

29. Kestle J, Drake J, Milner R, et al. Long-term follow-up data from the Shunt Design Trial. Pediatr Neuresurg. 2000; 33(5):230-236.

30. Huppert FA, Brayne C, Gill C, Paykel ES, Beardsall L. CAMCOG-a concise neuropsychological test to assist dementia diagnosis: socio-demographic determinants in an elderly population sample. Br J Clin Pyodol. 1995;34(pt 4):529-541.

31. Nasreddine ZS, Phillips NA, Bedirian V, et al. The Montreal Cognitive Assessment, MoCA: a brief screcning tool for mild cognitive impairment. $/ \mathrm{Am}$ Geriatr Sac. 2005:53(4):695-699.

32. Pinto FC, da Cunha Neto MB, Rocha MG, do Lago DV, Bronstein MD, Teixeira MJ. Hypopituitarism due to hydrocephalus: case report and review of the literature. Pediatr Neturosary. 2011;47(4):303-306.

\section{COMMENTS}

The authors should be complimented for having provided the neurosurgical community with the preliminary results of this perspective randomized clinical trial aiming to clarify which is the best surgical option between endoscopic third ventriculostomy (ETV) and ventriculo-peritoneal shunt (VPS) for the treatment of idiopathic normal pressure hydrocephalus (INPH). We really need well-designed randomized prospective studies to shed light on a disease where Level 1 evidences that favor surgical treatment are still lacking ${ }^{1}$ and patient selection is still extremely variable and debated.

This is not surprising, since even today the actual physiopathological basis of the disease are not yet completely understood and certain diagnosis is further complicated by the variability in the clinical presentation and course. 
PINTO ET AL

We fully agree with the authors that chose and applied strict inclusion criteria in order to obtain the most homogeneous population possible. As a consequence of this, out of 90 patients screened, only 42 were included. This supports the general feeling that multicenter trials involving big caseload centers need to be designed.

This study again confirms that ETV is able to obtain a kind of therapeutic result in some of INPH patients, thus stressing how complicate is the issue of cerebrospinal fluid (CSF) circulation and reabsorption, how simplistic is the classification of communicating and non-communicating hydrocephalus, and, once again, our lack of a complete understanding of the physiopathology of this disease. In addition, what further complicates the issue of correct surgical indication in $\mathrm{NPH}$ is patient selection and recognition of similar dinical entities such as long-standing overt ventriculomegaly of adulthood-LOVA ${ }^{2}$ in which the hydrocephalus is invariably due to acqueductal stenosis and for which ETV appears as the best form of treatment, when needed (i.e. decompensated patients).

The main limitations of this study are represented by the kind of tests used for patients selection, the tap test (TT), the small sample and the use of nonprogrammable valves, although there is consensus that TT alone has low sensitivity ${ }^{3}$ and the implantation of programmable VPS seems to be beneficial in the management of INPH.
Finally, the best functional outcome at 1 year follow-up obcained by VPS reinforces its role in the treatment of true INPH and limits the indication of ETV to a subgroup of patients that still requires to be clearly identified.

Paolo Ferroli

Morgan Broggi

Milan, Italy

1. Toma AK, Papadopoulos MC, Stapleton S, Kitchen ND, Watkins LD. Conservative versus surgical mamagement of idiopathic normal pressure hedrocephales: ized controlled trial: study protocol. Acta

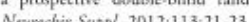

2. Oi S, Shimoda M. Shibara M. et al. Pathophysiology of long-standing wert ventriculomegaly in adults. / Neuresang. 2000;92(6):933-940.

3. Marmarou A. Bergsneider M. Klinge P. Rellkin N. Black PM. The value of supplemental prognostic teses for the properative assessment of idioparthic normalpressure hydrocephalus. Newrosurgery. 2005;57(3 suppl):517-S28; discussion it- $v$ 4. Bergsncider M, Black PM, Klinge P, et al. Surgical management of idiopathic normal-pressure hydrocephalus. Neurossugery. 2005:57(3 Suppl):S29-S39; discussion ii-v.

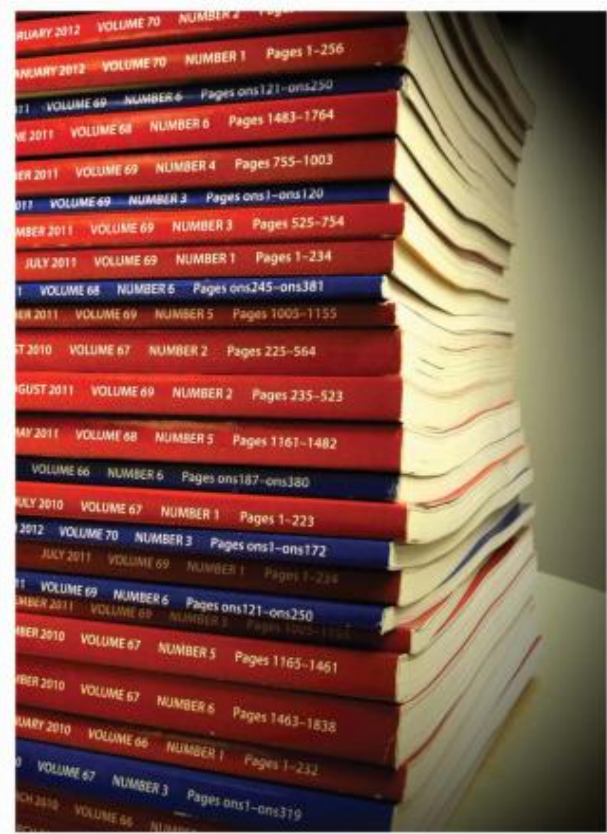

\section{Discuss.}

NEUROSURGERY® Journal Club.

This new feature capitalizes on and extends the existing practice of Journal Club common to all neurosurgical training programs where resident and fellows critically review published articles under the guidance of faculty.

For more information, please contact the Neurosurgery Editorial Office by phone at 404.712.5930; or email managingeditor@1cns.org

\section{NEUROSURGERY}

